\title{
THE MIND AND THE PHYSICAL WORLD \\ A Psychologist's Exploration of Modern Physical Theory
}

Douglas M. Snyder

\author{
Tailor Press \\ Los Angeles, California
}

Copyright 1995, 1996 Douglas M. Snyder 
Copyright 1995, 1996 by Douglas M. Snyder

All rights reserved

“Figures with Waves” by Ruth C. Snyder

Tailor Press

P. O. Box 18310

Beverly Hills, California 90209-4310

ISBN 0-9653689-0-4

Printed in the United States of America 


\section{Table of Contents}

\section{CHAPTER 1}

INTRODUCTION.......................................................... 1

\section{CHAPTER 2}

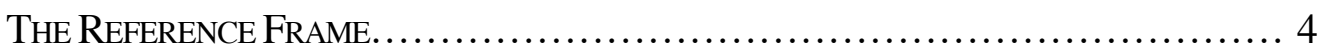

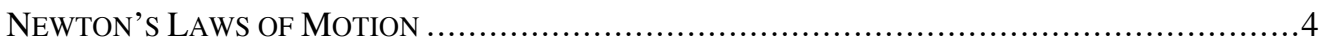

GALILEAN RELATIVITY ...................................................................

The Invariance of Newton's Second Law ...........................................................

SPATIAL LENGTH AND TEMPORAL DURATION

IN INERTIAL REFERENCE FRAMES......................................................

Absolute Space and Time .......................................................... 8

BEING AT REST ......................................................................... 13

The Significance of the Observer's Being

at Rest in a Reference Frame................................................... 14

A CONSEQUENCE OF THE COGNITIVE NATURE OF THE

REFERENCE FRAME IN THE CONSERVATION OF ENERGY,

LineAR MOMENTUM, AND ANGULAR MOMENTUM......

The Interchangeability of Physical Action and Cognition

in the Linear Translation of a Physical System.......................................... 17

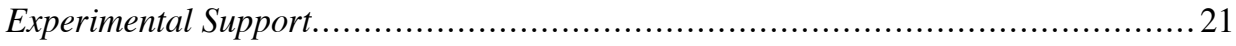

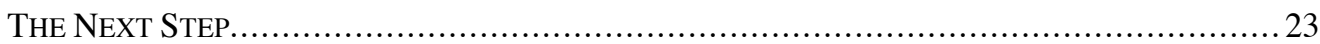

\section{CHAPTER 3}

THE SPECIAL THEORY OF RELATIVITY ........................................ 24

EINSTEIN'S 1917 ARGUMENT ON THE

RELATIVITY OF SIMULTANEITY ....................................................... 27

Switching Which Reference Frame is "Stationary"

and Which is "Moving" ....................................................... 30

THE ARBITRARY DECISION IN THE ARGUMENT ON THE

RELATIVITY OF SimUlTANEITY AND SOME CONSEQUENCES................................ 34

The Spatial Length Along the Axis of Motion of

Physical Existents at Rest in Either the "Stationary"

or "Moving" Reference Frame ................................................... 34

THE TIME OF THE "STATIONARY” INERTIAL REFERENCE FRAME ............................... 41

The Rate of Clocks at Rest in Either the "Stationary"

or "Moving" Reference Frame ................................................ 42

THE REFERENCE FRAME IN THE SPECIAL THEORY .......................................... 47 


\section{Contents}

THE SigNIFICANCE OF THE OBSERVER's BEING AT REST IN AN INERTIAL REFERENCE FRAME

IN THE SPECIAL THEORY

\section{CHAPTER 4}

The General Theory of Relativity...................................... 50

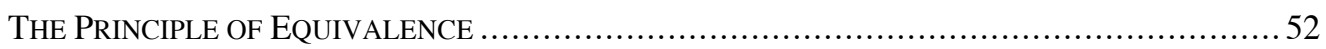

The Equality of Inertial and Gravitational Mass .............................................5 54

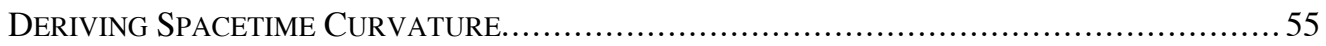

Further Comments on Extending the Results

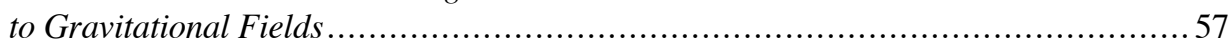

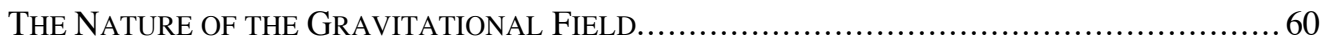

THE SIGNIFICANCE OF THE OBSERVER's BEING AT REST

IN A REFERENCE FRAME IN THE GENERAL THEORY ...................................................... 64

\section{CHAPTER 5}

QUANTUM MECHANICS ................................................. 67

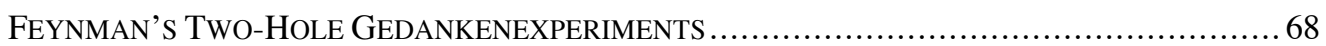

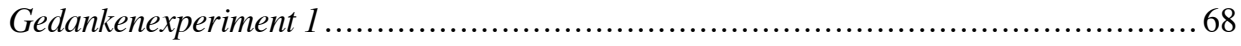

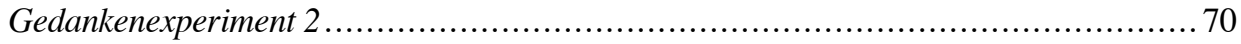

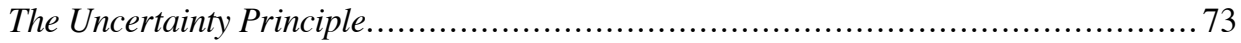

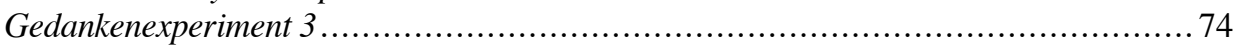

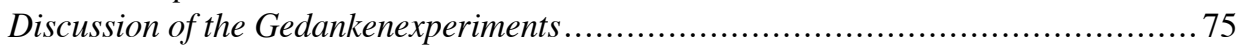

THE SCHRÖDINGER CAT GEDANKENEXPERIMENT ............................................ 79

KNOWLEDGE AND THE MEASUREMENT OF THE SPIN

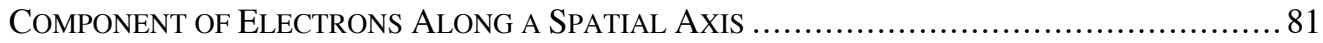

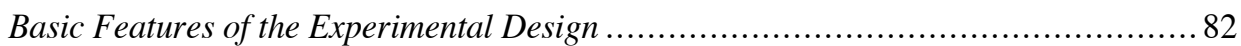

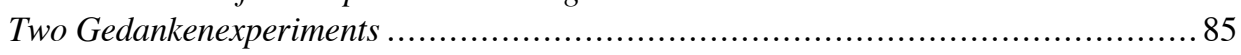

A Variation of the Gedankenexperiments .............................................. 90

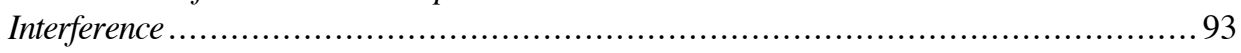

Another Indication of the Importance of Knowledge

in Measurement in Quantum Mechanics....................................................... 94

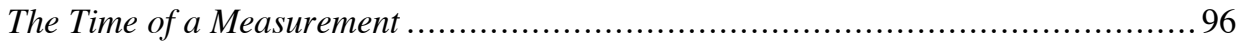

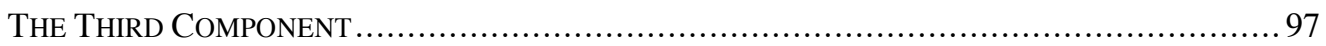

\section{CHAPTER 6}

StATISTICAL MECHANICS............................................. 98

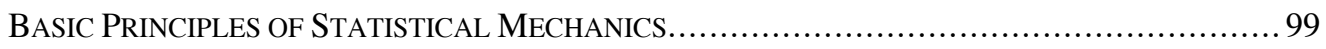

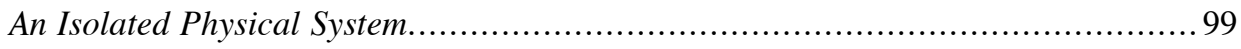

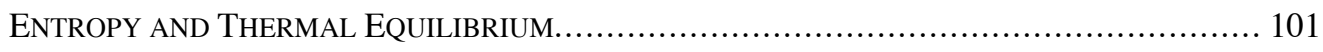

An Example of the Tendency to Thermal Equilibrium...................................... 104 


\section{Contents}

THE SignificANCE OF THE Fundamental ASSUMPTION ......................................... 106

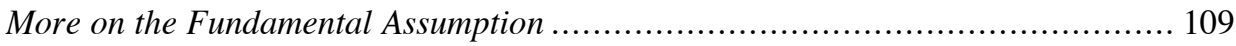

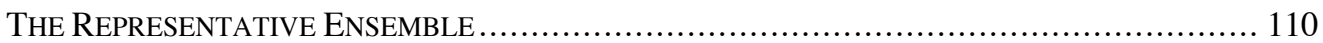

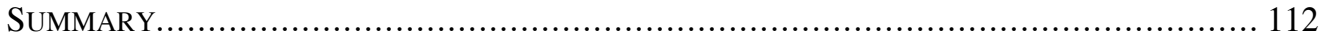

\section{CHAPTER 7}

CAn Psychology Make Its Own Contribution? ......................... 114

THE EFFECT OF INVERSION OF INCOMING LIGHT ON

VISUAL PERCEPTION AND VISUALLY GUIDED BEHAVIOR ...................................... 115

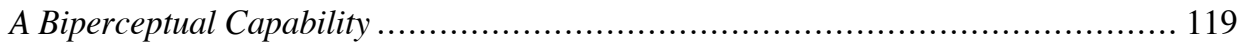

Hard Wiring of the Visual System and the Isotropy of Space ............................ 122

MANiPUlating Visual PERCEPTION IN THE

INVESTIGATION OF A PHYSICAL SYSTEM......................................................... 123

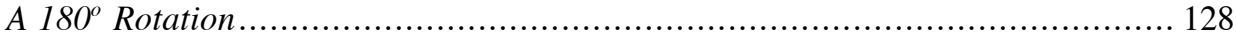

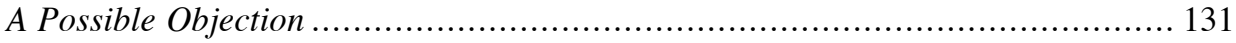

SUMMARY OF THE RESULTS OF THE GEDANKENEXPERIMENTS ............................... 132

\section{CHAPTER 8}

CONCLUSION......................................................... 134

\section{APPENDICES}

A - The AsSOciation of LENGTH AND DURATION WITH THE LOGICAL ROLES OF INERTIAL REFERENCE FRAMES IN THE ARGUMENT ON THE RELATIVITY OF SiMULTANEITY .................. 138

B - The NATURE OF $C+V$ AND $C-V$ IN THE Special Theory................. 142

C - The Light Flashes Used to Define Simultaneity in INERTIAL REFERENCE FRAMES IN THE SPECIAL THEORY .................... 144

D - Light Clocks AND Non-Light Clocks ................................. 147

E - The Mathematical Representation of Thermal Equilibrium .......... 151

F - TOLMAN'S ARGUMENT CONCERNING THE EQUIVALENCE of Time AND ENSEMBLE AvERAGES ................................ 152

REFERENCES ................................................. 155 


\section{Chapter 1}

\section{Introduction}

The mind of man is central to the structure and functioning of the physical world. Modern physical theory indicates that the mind stands in a relationship of equals to the physical world. Both are fundamental, neither can be reduced to the other, and both require each other for their full understanding.

This thesis is at odds with the view of the universe found in Newtonian mechanics as well as the generally held view among contemporary physicists of modern physical theory. Since the Renaissance, man has come to understand a great deal about the physical world, and he has gained significant control over it. This increased power over the physical world has occurred hand in hand with the assumption that the structure and functioning of the physical world is essentially independent from his cognitive functioning. According to this assumption, if man's cognitive capacity did not exist, the functioning of the physical world would not be fundamentally altered.

This last statement is not in fact correct, and modern physical theory, and even fundamentals underlying Newtonian mechanics, provide evidence to attest to this. Nonetheless, contemporary physicists for the most part do not see that the relationship of human cognition to the physical world is radically altered in their own modern theory, theory that is supported by a great deal of empirical data. Instead, attempting to preserve the thesis that the structure and functioning of the physical world is independent of the mind while on a practical level relying on modern theory that contradicts this thesis, physicists have placed themselves in the position of wondering at times exactly what is the nature of the physical world at the same time they obtain experimental results concerning the physical world that can only be labeled astonishing in their precision and the scope of their implications. ${ }^{1}$

\footnotetext{
${ }^{1}$ Historically, the attempt to link cognition and the physical world in modern physical theory has been minimal. In the area of quantum mechanics, a few physicists, such as Wigner (1961/1983) at Princeton, have over the years proposed that consciousness may unavoidably affect the physical world. But these physicists have not been thorough in their approach to the issue, and their ideas in general have not been well received in the physics community.

The features of quantum mechanics that have supported Wigner and other like-minded physicists generally have been treated as curiosities. Most physicists have not considered them as indications that a realistic view of the physical world, independent of the thinking, observing person, is invalid. These physicists in large part employ the quantum mechanical
} 


\section{Chapter 1}

Modern physical theory consists of three main components: 1) the special and general theories of relativity; 2) quantum mechanics; and 3) statistical mechanics. There are very successful theories that have been developed on the basis of these three bedrock areas. An example of one is quantum electrodynamics. But these theories owe their conceptual foundation to the three components mentioned. The basic issues at the core of these three components also are expressed in these later theories. In addition, there are new unresolved issues of a fundamental nature concerning the conceptual integrity of these later theories that do not apply to quantum mechanics, relativity theory, and statistical mechanics.

Quantum mechanics and relativity theory are areas I have written about for over twelve years. The nature of statistical mechanics has also been of interest to me during this time. But when I took a serious look in 1993 at Tolman's (1938) The Principles of Statistical Mechanics, it became clear that the mind is linked to the physical world in statistical mechanics, a relationship I had found earlier in both relativity theory and quantum mechanics. It was after reading Tolman's justification of the method of statistical mechanics in the original that I decided to write this book. When I found that the three components of modern physical theory all pointed to the same relationship between mind and the physical world, it became clear that the fundamental isolation of the mind from the physical world that has characterized our experience since the development of Newtonian mechanics is unfounded. Based on empirically supported principles of modern physical theory, I determined that the appropriate assumption for one's experience, that the mind is linked to the physical world, could be stated with confidence.

The impact of this change in assumption concerning the relationship of man to the cosmos in modern physical theory will find its way into our everyday experience. It will perhaps have no greater effect than in reducing the sense of isolation of man from the world that has characterized modern existence.

formalism in a practical way without focusing on the implications of the formalism (Mermin, 1985).

Recently, there is growing interest in the implications of modern physical theory as regards the relationship between human cognition and the physical world. Much of this early interest has been in the area of quantum mechanics (e.g., Goswami, 1989; Snyder, 1983, 1986, 1989). 


\section{Introduction}

The plan of this book is as follows. First, the nature of the reference frame in physics will be considered. Second, the special and general theories of relativity will be discussed. Third, quantum mechanics, and then statistical mechanics, will be explored. Fourth, an example of how psychology can make its own contribution in delineating the nature of the link between the mind and the physical world is presented. Appendices contain supporting information for some of the arguments presented. 


\section{Chapter 2}

\section{The Reference Frame}

Indications of the importance of mind to the physical world can be found in the most central features of physical theory, modern or Newtonian. These indications center on the nature of the reference frame and its pivotal role in physical theory. The motion of a physical body requires conceptions of both space and time. Further, and very importantly, the motion of a physical body requires a vantage point from which to judge it. This vantage point is basically a spatial coordinate frame, attached to some other physical body, which can be used to assign spatial coordinates to the body being studied at various times. It is called a frame of reference. Not only is a method of assigning spatial coordinates required, but a method of assigning temporal coordinates is needed. These temporal coordinates are assigned with the aid of clocks.

\section{Newton's Laws of Motion}

Newton's laws of motion, of course, have been found to be supported by a great deal of experimental evidence. But these laws do not hold in all frames of reference. These laws, for example, do not hold in an accelerating reference frame.

Newton's first law of motion states that in the absence of the application of an external force, a physical body either is at rest or maintains a uniform, rectilinear motion. A frame of reference in which Newton's first law holds is called an inertial frame of reference. Further, with some mathematics, it can be established that if Newton's first law holds in one frame of reference, it should hold in any frame of reference in uniform translational motion (i.e., at a uniform speed in a straight line) relative to this frame. A railway train and the embankment on which it is traveling in uniform translational motion are idealized examples of inertial reference frames.

With a bit more mathematics, the Galilean principle of relativity can be established. This principle states: If Newton's laws hold in one frame of reference, they will hold in another reference frame in uniform translational motion relative to the former frame (Einstein, 1917/1961). Using the space and time coordinate transformations for inertial reference frames which underlie the Galilean principle of relativity, one can derive that the lengths of physical bodies and the duration of physical occurrences are the same in inertial reference frames in uniform translational motion relative to one another. 


\section{The Reference Frame}

Galilean relativity is an important concept in a number of arguments indicating that the physical world does not exist independently of the observing and thinking individual. Therefore, the points in the preceding paragraph will be discussed in more detail.

\section{Galilean Relativity}

Consider two inertial reference frames $\mathrm{W}$ and $\mathrm{W}^{\prime}$, each with two spatial dimensions and oriented in space in the same manner $\left(x, y\right.$ for $\mathrm{W}$ and $x^{\prime}, y^{\prime}$ for $\mathrm{W}^{\prime}$ ) in uniform translational motion relative to one another and relying on the Galilean coordinate transformation for time

$$
t^{\prime}=t
$$

where $t$ applies to $\mathrm{W}$ and $t^{\prime}$ applies to $\mathrm{W}^{\prime}$, that are displayed in Figure 1. In these reference frames, used in Newtonian mechanics, the designation of one of the frames as "stationary" and the other as "moving" is inconsequential for Newton's laws of motion and the temporal duration of an occurrence or the spatial length of a physical existent in the inertial reference frames.

In Newtonian mechanics, for $\mathrm{W}$ and $\mathrm{W}^{\prime}$ in uniform translational velocity relative to one another along the $x$ and $x^{\prime}$ axes, respectively, the Galilean transformation equations for the $x$ and $x^{\prime}$ axes are

$$
x^{\prime}=x-v t
$$

and

$$
x=x^{\prime}+v t^{\prime} .
$$

As noted, the time relation is $t^{\prime}=t$. Eqn. 2 relates $x^{\prime}$ in $\mathrm{W}^{\prime}$ to $x$ in $\mathrm{W}$ over time where $\mathrm{W}^{\prime}$ is considered moving at a constant speed in a straight line relative to $\mathrm{W}$. W is considered "stationary" and $\mathrm{W}^{\prime}$ is considered "moving." In Eqn. 2, the $x^{\prime}$ axis is considered moving relative to the $x$ axis using the time $t$ associated with the $x$ axis to determine this motion. Eqn. 3 relates $x$ in $\mathrm{W}$ to $x^{\prime}$ in $\mathrm{W}^{\prime}$ over time where $\mathrm{W}$ is considered moving at a constant speed in a straight line relative to $\mathrm{W}^{\prime}$. W' is considered "stationary" and $\mathrm{W}$ is considered "moving." In Eqn. 3 , the $x$ axis is considered moving relative to the $x^{\prime}$ axis using the time $t^{\prime}$ associated with the $x^{\prime}$ axis to determine the motion of W. Eqn. 2 transforms to Eqn. 3 simply in the following way. From Eqn. 2

$$
x=x^{\prime}+v t .
$$

Through substitution from $t^{\prime}=t$

$$
x=x^{\prime}+v t^{\prime} \text {. }
$$




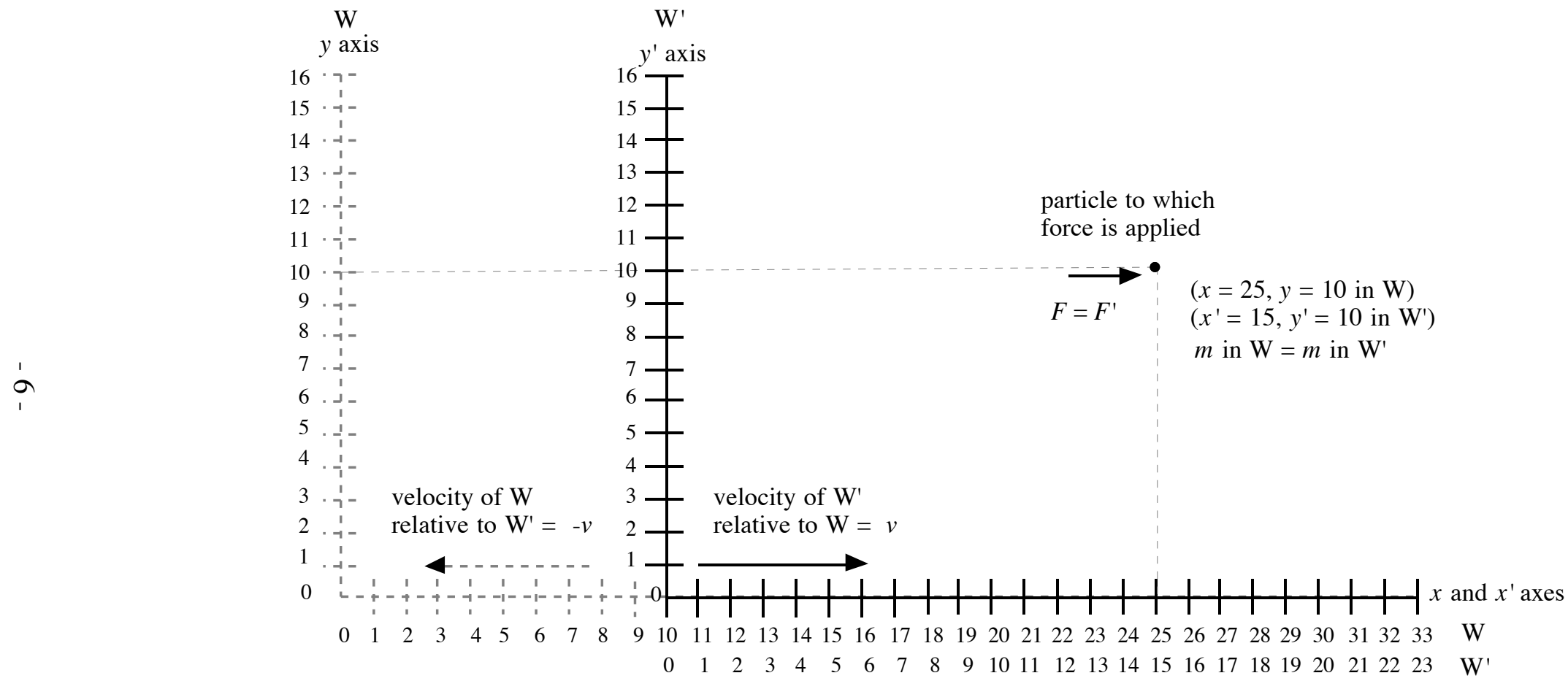

Figure 1

A particle considered in two inertial reference frames of two spatial dimensions in uniform translational velocity relative to one another. 


\section{The Reference Frame}

The Invariance of Newton's Second Law

As a demonstration of the Galilean principle of relativity, the invariance of Newton's second law will be shown. Taking the derivative of each side of Eqn. 2 with respect to the time $t$, one obtains

$$
d x^{\prime} / d t=d x / d t-d(v t) / d t
$$

and

$$
d x^{\prime} / d t=d x / d t-v
$$

because $v$ is constant. Substituting $t^{\prime}$ for $t$ on the left side of the above equation

$$
d x^{\prime} / d t^{\prime}=d x / d t-v .
$$

Letting $d x^{\prime} / d t^{\prime}=u^{\prime}$ and $d x / d t=u$

$$
u^{\prime}=u-v \text {. }
$$

Now

$$
d u^{\prime} / d t=d u / d t-d v / d t .
$$

As $v$ is constant and by substituting $t^{\prime}$ for $t$ on the left side of the above equation

$$
d u^{\prime} / d t^{\prime}=d u / d t
$$

and

$$
a^{\prime}=a
$$

where $a^{\prime}=d v^{\prime} / d t^{\prime}$ and $a=d v / d t$. Since $m$ is constant in both $\mathrm{W}$ and $\mathrm{W}^{\prime}$, and since the general formulation of Newton's second law of motion is $F=m a$ where $F$ is the external force applied to an object and $a$ is the resulting acceleration in the direction of application of the external force, it can be concluded that $F=m a$ in $\mathrm{W}$ and $F^{\prime}=m a^{\prime}$ in $\mathrm{W}^{\prime}$. Thus, Newton's second law is invariant in inertial reference frames in uniform translational motion relative to one another. The argument can be made using Eqn. 3 with $\mathrm{W}$ considered moving relative to $\mathrm{W}^{\prime}$, and the result will be the same.

\section{Spatial Length and Temporal Duration in Inertial Reference Frames}

In Newtonian mechanics, from the time relation $t^{\prime}=t$, clearly the duration of an occurrence in the two inertial reference frames is the same. If $t_{2}$ and $t_{1}$ are the time boundaries of an occurrence in $\mathrm{W}$, then the time boundaries of the occurrence in $\mathrm{W}^{\prime}$ are the same because $t_{2}=t^{\prime}{ }_{2}$ and $t_{1}=t^{\prime}{ }_{1}$ where $t_{2}^{\prime}$ and $t_{1}^{\prime}$ are the time boundaries of the occurrence in $\mathrm{W}^{\prime}$ (Figure 2). Thus 


\section{Chapter 2}

$$
\Delta t^{\prime}=\Delta t
$$

where $\Delta t=t_{2}-t_{1}\left(t_{2}>t_{1}\right)$ and $\Delta t^{\prime}=t_{2}^{\prime}-t_{1}^{\prime}\left(t_{2}^{\prime}>t_{1}^{\prime}\right)$.

It follows from the time relation, $t^{\prime}=t$, that the length of an object along the $x$ and $x^{\prime}$ axes in the two reference frames is the same (Figure 3). From Eqn. 2, it follows that

$$
\Delta x^{\prime}=\Delta x-\mathrm{v} \Delta t
$$

where $\Delta x^{\prime}=x_{2}^{\prime}-x_{1}^{\prime}\left(x_{2}^{\prime}>x_{1}^{\prime}\right), \Delta x=x_{2}-x_{1}\left(x_{2}>x_{1}\right)$. If $\Delta t=0$,

$$
\Delta x^{\prime}=\Delta x
$$

and, in general

$$
\left|\Delta x^{\prime}\right|=|\Delta x| .
$$

When $\Delta t=0,|\Delta x|$ represents the length of the object in $\mathrm{W}$ as the two ends of the object are measured at the same time in $\mathrm{W}$. Because $\Delta t^{\prime}=\Delta t$, when $\Delta t=0$, $\Delta t^{\prime}=0$. Thus $\left|\Delta x^{\prime}\right|$ is the length of the object in $\mathrm{W}^{\prime}$ when $|\Delta x|$ is the length of the object in W. Using Eqn. 3, one finds in the same manner that

$$
|\Delta x|=|\Delta x| \text {. }
$$

\section{Absolute Space and Time}

Notice that though $t^{\prime}=t$ indicates that time is absolute in the Galilean transformation, Eqns. 2 and 3 do not indicate that space is absolute. Absolute space refers to space that is independent of the particular reference frame employed by observers in describing the motion of some physical body. Indeed, Eqns. 2 and 3 indicate that an observer requires a reference frame, in this case an inertial reference frame, from which to determine the motion of objects. That lengths of objects are equal in inertial reference frames in uniform translational motion relative to one another in Newtonian mechanics supports the view that space may be absolute in Newtonian mechanics. That lengths of objects in such reference frames are equal does not imply that space must be absolute.

For example, if one inertial reference frame is as good as another inertial reference frame in explaining the motion of some physical body, is there any inertial reference frame which can be considered an accurate representation of absolute space? Newton, nonetheless, maintained that absolute space indeed exists. In the Principia, he wrote: 


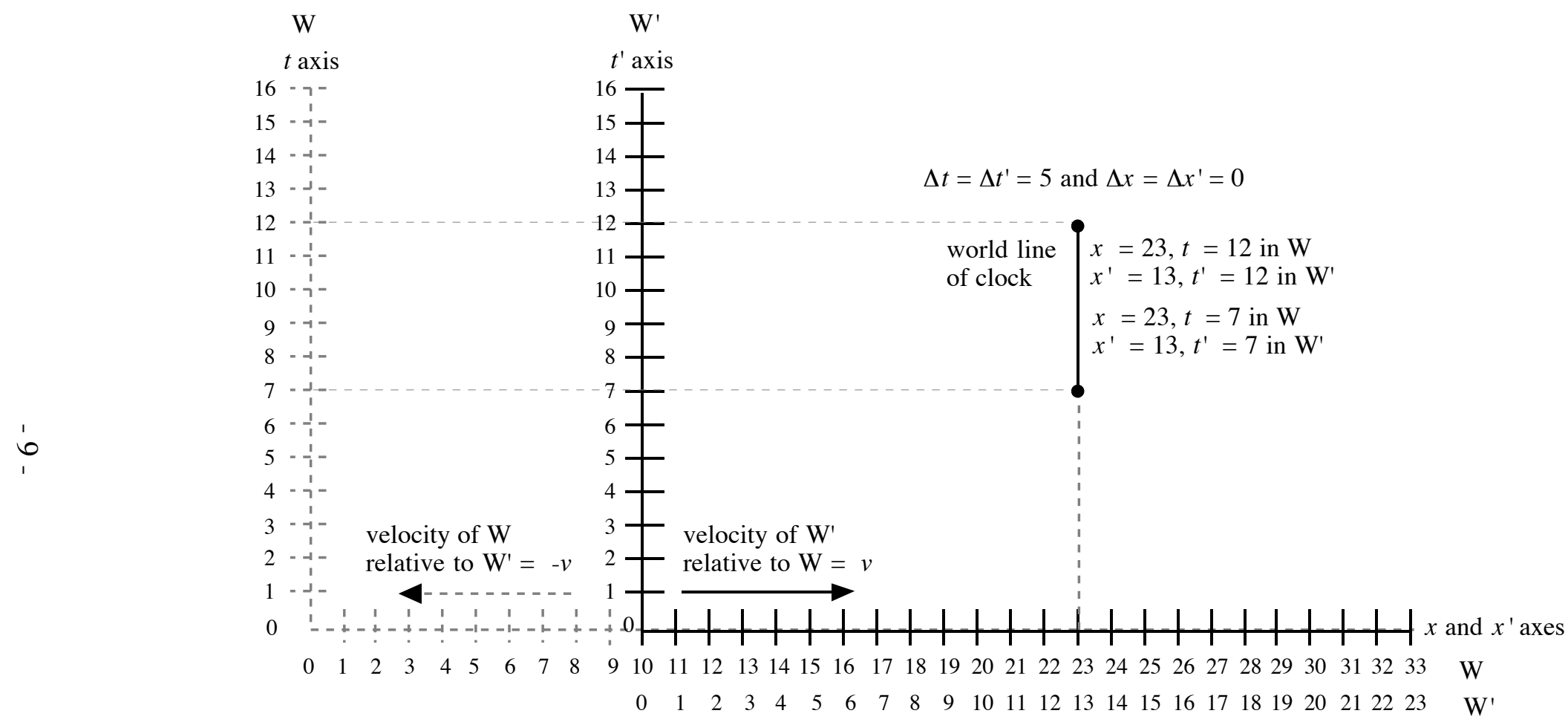

Figure 2

The duration of an occurrence in two inertial reference frames $\mathrm{W}$ and $\mathrm{W}^{\prime}$ in uniform translational motion relative to one another with the occurrence happening at the same location along the $x$ and $x^{\prime}$, and $y$ and $y^{\prime}$, spatial axes of $\mathrm{W}$ and $\mathrm{W}^{\prime}$, respectively. The $y$ and $y^{\prime}$ axes are not shown. 


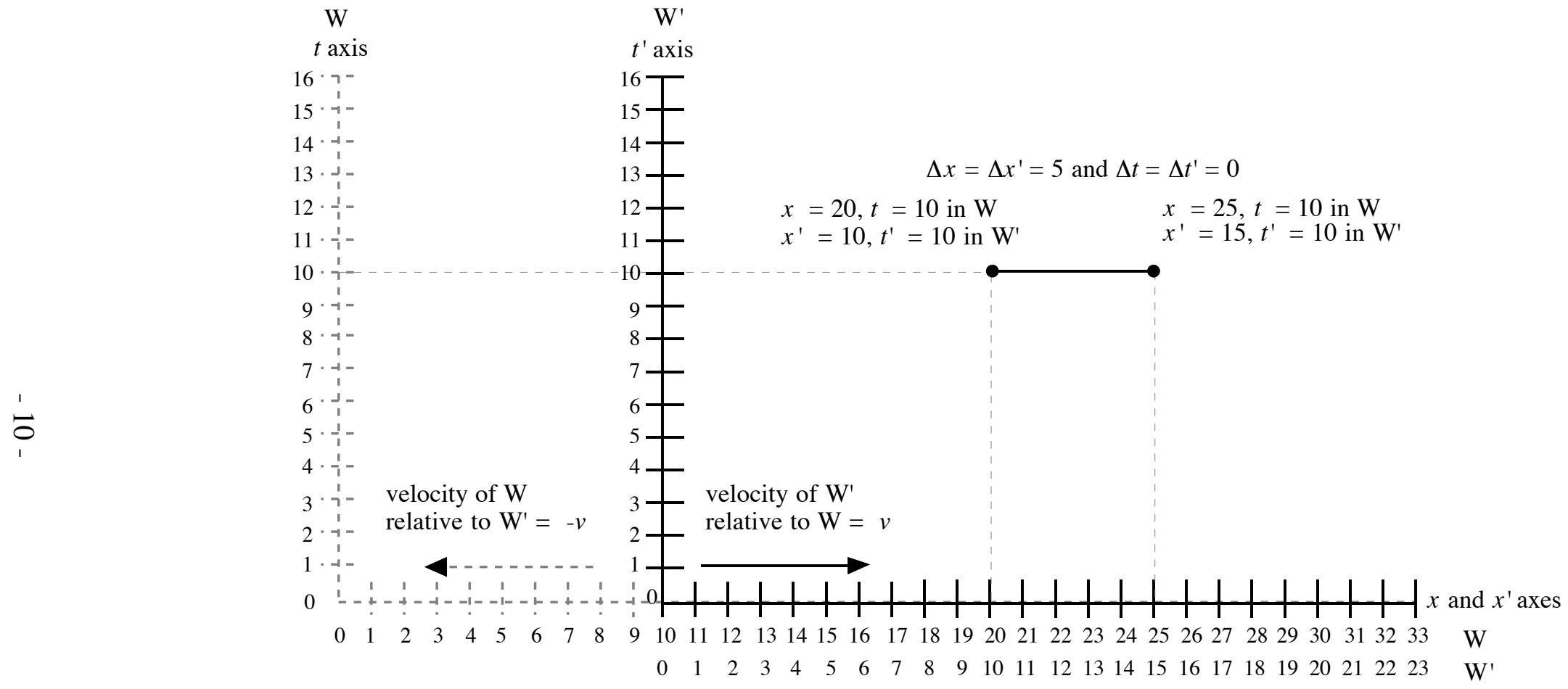

Figure 3

The length of an object in two inertial reference frames $\mathrm{W}$ and $\mathrm{W}^{\prime}$ in uniform translational motion relative to one another with the object aligned along the $x$ and $x^{\prime}$ spatial axes of $\mathrm{W}$ and $\mathrm{W}^{\prime}$, respectively. The $y$ and $y^{\prime}$ axes are not shown. 


\section{The Reference Frame}

Absolute space, in its own nature, without relation to anything external, remains always similar and immovable. (Newton, $1686 / 1962$, p. 8)

And what about time? Absolute time is at the heart of Newton's laws: it is absolute time that allows Newton's laws to hold in inertial reference frames in uniform translational motion relative to one another. Operationally, by absolute time, it is meant that the temporal coordinates assigned to the motion of some body in one inertial reference frame may also be used in describing the motion of this body from another inertial reference frame. Newton described absolute time as:

Absolute, true, and mathematical time, of itself, and from its own nature, flows equably without relation to anything external. (Newton, 1686/1962, p. 6)

If absolute space exists, the importance of particular frames of reference with their attendant observers from which to describe the motions of physical bodies is minimal. Even though the same spatial coordinate values describing the motion of physical bodies cannot be assigned in different inertial reference frames in uniform translational motion relative to one another, the tremendous empirical support for Newton's laws over two centuries made it appear until the early twentieth century that physicists understood the nature of space and time.

There is no mention of mind in Newton's characterization of absolute space and time. Absolute time and absolute space exist "without relation to anything external" (Newton, 1686/1962, pp. 6, 8). Kant, on the other hand, maintained that mind employs a priori forms of space and time in the sensuous experience of the physical world (Kant, 1781/1966). Nothing in Newton's laws of motion indicates that Kant is incorrect. As will be shown, the development of physical theory in special and general relativity (and the empirical evidence that supports these theories) indicates not only that space and time are not absolute, but also that Kant's own view of a priori categories of space and time are inadequate to account for the variety of forms of space and time allowed by special and general relativity.

Further, in their consideration of the physical world, physicists ultimately rely on empirical evidence to determine whether or not their theories are correct. This evidence takes the form of measurements, and in the case of describing the motion of physical bodies it involves measurements of spatial length and temporal duration. It is the physicist who, in adopting particular 


\section{Chapter 2}

conceptions of space and time, guides the construction and application of various measuring instruments and interprets the results obtained with those instruments.

When the tremendous success of Newtonian mechanics is considered in isolation, it appears possible that time and space may be wholly explained in terms of the physical world without any contribution on the part of a theorizing or observing individual. But, as will be shown, one is confronted with a good deal of evidence that the temporal and spatial structure of the physical world involves particular conceptions of space and time (or spacetime) that are applied by observers to the physical world.

Even in Newtonian mechanics, notwithstanding the control over nature achieved with this theory in which the absolute nature of time and the quasiabsolute nature of space is assumed, the Galilean principle of relativity underlying these mechanics must be accounted for. Even more fundamentally, the nature of the reference frame and its associated temporal structure have not been altered by the particular results that have been achieved in the Newtonian mechanics that relies on them, including the invariant spatial length of physical existents or the invariant temporal duration of occurrences. In general, the reference frame and its associated temporal coordinate system that are used by an observer are not independent of anything external, but instead are in part inventions of the human mind. The use of a reference frame, or a temporal coordinate system, involves the application of categories of space and time to the physical world, though these categories are not limited in their expression to those absolute ones proposed by Kant.

Natural scientists from the late seventeenth through the nineteenth centuries could plausibly maintain that space and time are absolute and thus independent of anything external. Though the possibility of absolute space is not precluded in Newtonian mechanics, the situation is different in relativity theory. The fundamental importance of the reference frame cannot be denied in the special theory of relativity because the nature of spacetime in this theory depends on the particular inertial reference frame from which the physical world is considered. This point concerning relativity theory will be discussed in the following chapters. Before investigating relativity theory, a closer look at the concept of an observer at rest in his reference frame that is implied in an observer's use of a reference frame to describe the motion of a physical body

will also indicate that mind is linked to the physical world. 


\title{
The Reference Frame
}

\author{
Being at Rest
}

When I was a boy, I had a favorite ride at the amusement park in Santa Monica. I would walk into a large cylinder along with other riders, and we would all stand with our backs against the inside wall of the cylinder. The cylinder would begin to rotate around its axis, and the speed of its rotation would increase until it was rotating very quickly. Then, the floor upon which we were standing would drop away.

Amazingly, we did not fall. In fact, we were glued to the cylinder wall. I felt glued to the wall by a force that was pushing me flat against it and the direction of which was along a line through me and which emanated from the center of the cylinder. I was restrained by the cylindrical wall itself, the counterforce being experienced as equal to the force pushing me outward. What held me up against the force of gravity was the force due to friction resulting from my body pressing against the wall of the cylinder.

For an observer located outside the ride and at rest, the analysis of the motion would be much different. This observer can be considered to be in an inertial reference frame. This observer's analysis would be that the direction of my velocity is always tangent to the rotating cylinder. My velocity would be like a piece of a spinning top that breaks off at the edge of the top. Like the rotating cylinder, the magnitude of the velocity is constant. But, unlike the piece of the spinning top, the direction of my motion is constantly altered due to a force applied to me by the cylinder wall that is responsible for my continual acceleration toward the cylindrical axis (i.e., a centripetal force).

In this analysis of the dynamics of my motion, the frictional force due to my body's pressing against the cylinder is also responsible for keeping me from falling. This observer at rest in an inertial reference frame would essentially maintain an explanation of the dynamics of my motion in accordance with Newtonian mechanics. On the other hand, my experience of the dynamics of my motion is similar to analyses of rotational motion maintained by Descartes, where a real outward directed force, for example, is considered responsible for the climbing of water up the inside of a rotating bucket (Cohen, 1981).

In the Newtonian analysis of rotational motion, this outward directed, centrifugal force is not essential. Only a centripetal force is required. A complete and accurate understanding of my motion on the amusement park ride is achieved without centrifugal force. Indeed, Newton's analysis of rotational motion, in conjunction with Newton's law of gravitation, is responsible for 


\section{Chapter 2}

remarkably accurate predictions concerning the motions of the planets around the sun. The question arises as to why I experienced this outward directed force when it appears that a complete, accurate, and economical explanation does not require it. Is this experience just an insignificant oddity, or can it be seen to reveal something basic about the nature of the physical world and its relation to human experience?

\section{The Significance of the Observer's}

\section{Being at Rest in a Reference Frame}

It is unlikely that this experience of what is called centrifugal force is insignificant. The experience in situations like the amusement park ride is too powerful, and these situations occur too frequently. What then can it tell us about the nature of the physical world and an individual's relation to it? The answer can be found in that which distinguishes between my experience of centrifugal force and the analysis of the observer who is stationary on the ground (Snyder, 1992a).

This distinguishing feature concerns the different reference frames of the respective observers. That is, my reference frame on the ride is the rotating cylinder and the reference frame of the stationary observer on the ground is an inertial reference frame. But why should these different reference frames be responsible for the differing analyses and different experiences of the situation? For example, why can't I simply consider myself in the stationary reference frame of the observer on the ground and thus get rid of the experience of centrifugal force? It might appear that one is dealing only with simply choosing one coordinate scheme rather than another from which to describe my motion. And there are certainly transformation rules for transforming the description of motion in one reference frame into the description in another.

The reason for the difficulty in achieving this change of reference frame is the natural inclination to see oneself as an observer at rest in his frame of reference. It is this characteristic of experience that is the basis for distinguishing the experiences and analyses of the different observers of my ride at the amusement park. As implied in the above description of my experience on the ride, the outward directed, centrifugal force is needed by me, or another observer in similar circumstances, to counterbalance the applied centripetal force. My experience of the counterbalancing forces is associated with my experience of being at rest. 


\section{The Reference Frame}

A fundamental aspect of the measurement of motion in the physical world is that the observer involved in this measurement is at rest in some frame of reference. It is a point only partly emphasized by physicists in their acknowledgment of the importance of a reference frame in determining the motion of physical objects. The reference frame, in part an invention of the observer using it, is itself problematic for physicists. There is no necessity that a particular observer adopt a particular reference frame. And without choosing a particular reference frame, the observer's measuring instruments are essentially useless.

But even more fundamental, from a psychological perspective, is the notion of rest for the observer because this rest is completely beyond physical analysis. This rest is not the rest referred to in Newton's first law of motion. This being at rest for an observer in a reference frame is distinct from the motion or rest of physical objects observed by this individual. If someone else, for example, sees me rotating on the amusement park ride, I nonetheless as an observer am at rest in my reference frame. For this other individual, the amusement park ride is rotating at the same speed as I rotate. Even if I am aware that I, as my body, am rotating at a constant speed, as an observer I consider myself at rest in a reference frame. I am fundamentally at rest even though I may be cognitively convinced that I am in motion.

This last point bears repeating. There is nothing in the physical world that serves as the basis for explaining the observer's being at rest for himself or herself in a reference frame. The observer's being at rest in a reference frame is a psychological characteristic, not an explicitly physical one. The observer's being at rest in a reference frame is a characteristic that follows the psyche, not the material physical world. It does not, for example, follow the physical object associated with the observer's reference frame. There is nothing particularly unique about this physical object that distinguishes it from other physical objects, except that for the observer it is not moving relative to him. Its significance in terms of its, and the associated reference frame's, being at rest is that it is not in motion relative to the observer in the reference frame. If the object to which the observer's reference frame is associated is put into motion relative to him, it is no longer associated with his reference frame as an anchor, so to speak, for this reference frame. Another suitable object is needed. Wherever I am, I am fundamentally at rest, even if I conclude that my body is moving. As will be shown, this conclusion is reinforced by the special and 


\section{Chapter 2}

general theories of relativity in which the observer's being at rest in his reference frame is an essential feature of the theories.

An anecdote serves to demonstrate the very basic nature of a person's experience of being at rest in a reference frame and, at the same time, the difficulty in accepting this fact. About two years ago, I was driving in a car with my eight year old niece about two weeks after she had taken her first trip by airplane. As we were riding, my niece asked out of the blue, "Why doesn't it feel like you're moving when you're in an airplane?" She also said, "You get on the airplane at one place and get off the airplane at another, but you don't feel like you've moved anywhere." My niece, like the rest of us, had difficulty squaring her experience that she was not in motion with her having traveled to a far off place. Her experience of not being in motion in the airplane essentially constituted her being at rest in an inertial frame of reference.

\section{A Consequence of the Cognitive \\ Nature of the Reference Frame in the Conservation of Energy, Linear Momentum, and Angular Momentum}

The cognitive component of a reference frame and its associated temporal coordinate system is also embedded in the conservation laws of energy, linear momentum, and angular momentum (Snyder, 1985). Each of these conservation laws reflects an underlying invariance of physical law under some transformation of an isolated physical system, defined as a system that does not interact with any other physical system (Liboff, 1993). In the case of energy, the transformation is with regard to time. In the case of linear momentum, the transformation is linear spatial displacement of an isolated physical system. And in the case of angular momentum, the transformation is angular spatial displacement (i.e., rotation) of an isolated physical system. In demonstrating the invariance of physical law under one of these transformations, one does not have to act on the system itself. Rather, one can alter the relevant spatial or temporal axis used in the description of the system and effect the transformation. And rather than act on the physical objects themselves, such as rulers and clocks, one can simply alter the axis through the use of mathematics.

For example, consider the act of moving a physical system along an axis $x$ representing a linear spatial direction from $x_{1}$ to $x_{2}\left(x_{2}>x_{1}\right)$. This act is equivalent to leaving the system untouched and linearly moving the axis such 


\section{The Reference Frame}

that the coordinate of the system along this axis changes from $x_{1}$ to $x_{2}$. And moving a measuring device representing this axis is equivalent to adding $\left(x_{2}-x_{1}\right)$ to all points on the axis and leaving the measuring device untouched. Thus, the invariance of physical law under the spatial displacement of an isolated physical system, to be shown soon, is dependent on cognition, through the use of mathematics, to the same extent that it is dependent on the physical displacement of the system. This same interchangeability of moving the system itself or leaving the system untouched and changing the inertial frame of reference from which it is described also applies to the conservation of linear momentum. ${ }^{2}$

\section{The Interchangeability of Physical Action and}

\section{Cognition in the Linear Translation of a Physical System}

An example of the relationship between the invariance of physical law and a conservation law can be demonstrated as follows.

Invariance of Newton's Second Law of Motion

Consider the inertial reference frames $\mathrm{W}$ and $\mathrm{W}^{\prime}$. Only now, let $\mathrm{W}$ and $\mathrm{W}^{\prime}$ be at rest relative to one another and situated as in Figure 4 . Let there be a physical system located at spatial location $x_{2}=15$ at time $t_{2}$ in $\mathrm{W}$ on which an external force $F$ is exerted along the $x$ axis in the direction of increasing $x$. Let this system be located at $x_{2}^{\prime}=25$ in $\mathrm{W}^{\prime}$ at time $t_{2}^{\prime}$, and the force in $\mathrm{W}^{\prime}$ be $F^{\prime}$ on the system. Let the system have momentum $p=m v$ in $\mathrm{W}$ and $p^{\prime}=m v^{\prime}$ in $\mathrm{W}^{\prime}$. According to Newton's second law of motion, $F=m a(7)$ or $F=m(d v / d t)$ where

$$
v=\left(x_{2}-x_{1}\right) /\left(t_{2}-t_{1}\right) \text { in the limit }
$$

as $t_{2}-t_{1}\left(t_{2}>t_{2}\right)$ becomes very small for the motion of the system along the $x$ axis in $\mathrm{W}$. In $\mathrm{W}^{\prime}$, the velocity of the system is given as

$$
v^{\prime}=\left(x_{2}^{\prime}-x_{1}^{\prime}\right) /\left(t_{2}^{\prime}-t_{1}^{\prime}\right) \text { in the limit }
$$

with $\left(t_{2}^{\prime}>t_{1}^{\prime}\right)$ or

$$
v^{\prime}=\left[\left(x_{2}+\Delta x\right)-\left(x_{1}+\Delta x\right)\right] /\left(t_{2}-t_{1}\right) \text { in the limit. }
$$

\footnotetext{
${ }^{2}$ Indeed, changing reference frames is a purer way of demonstrating the invariance of physical law and the various conservation laws because the physical system itself is not affected directly and in principle the change in reference frame can occur instantaneously.
} 
reference frame shifted from $\mathrm{W}$ to $\mathrm{W}^{\prime}$

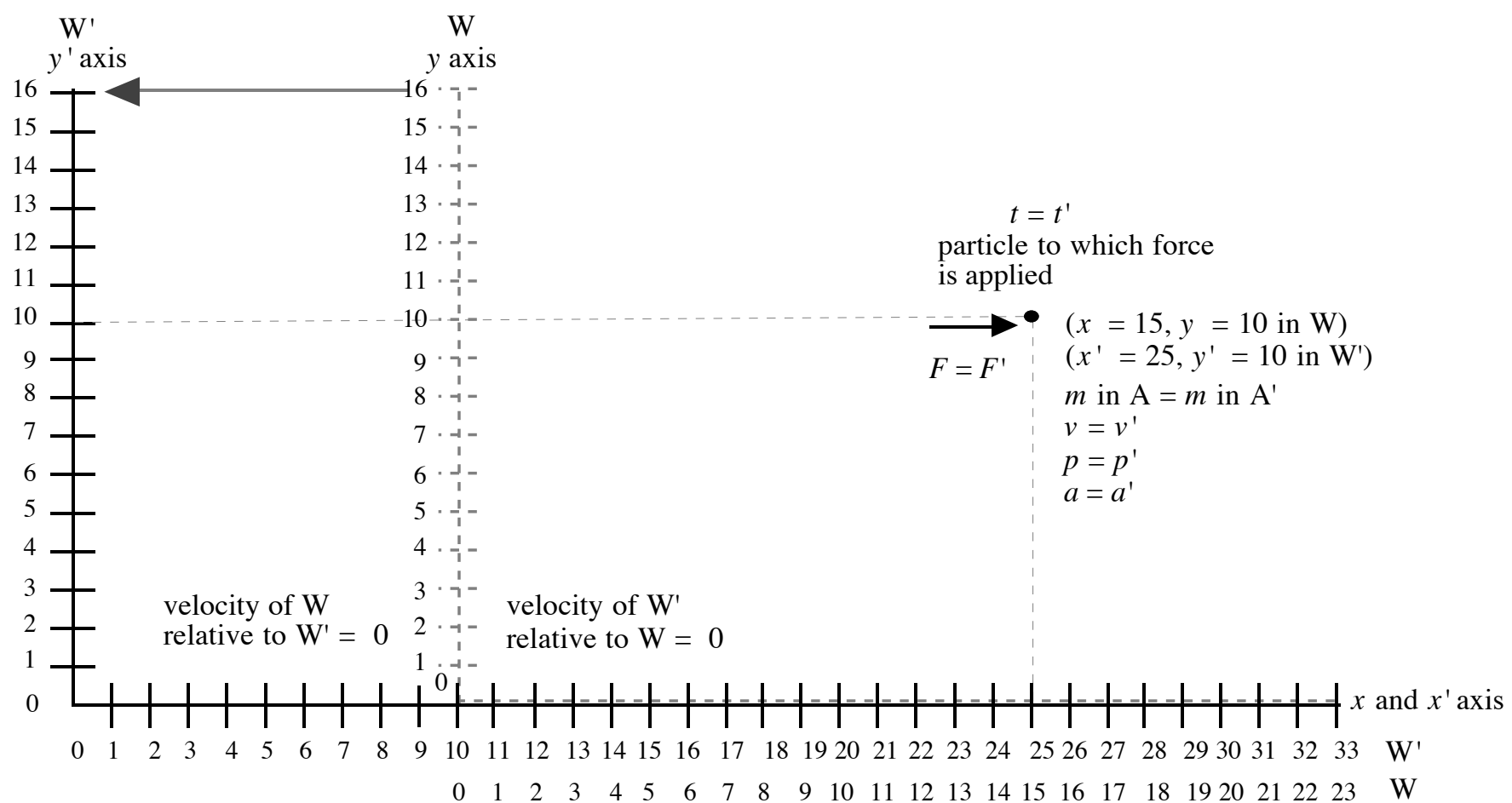

Figure 4

Linear translation of a physical system by shifting the inertial reference frame linearly with the effect on certain kinematic and dynamic quantities of the system shown. 


\section{The Reference Frame}

The right side of this equation becomes

$$
\left(x_{2}-x_{1}\right) /\left(t_{2}-t_{1}\right) \text { in the limit. }
$$

Thus, Eqn. 8 and Eqn. 9 are equivalent and $v=v^{\prime}$. As $m$ is constant in both of the inertial reference frames, as $t$ and $t^{\prime}$ are not affected by considering the system from $\mathrm{W}$ or $\mathrm{W}^{\prime}$ (i.e., $t=t^{\prime}$ ), and as velocity is unchanged in these considerations, Newton's second law remains valid in both $\mathrm{W}$ and $\mathrm{W}$ '. Since the velocities $v$ and $v^{\prime}$ of the system are the same, the accelerations of the system in $\mathrm{W}$ and $\mathrm{W}^{\prime}$ are also the same

$$
d v / d t=d v^{\prime} / d t^{\prime}
$$

or

$$
a=a^{\prime} .
$$

Then

$$
m d v / d t=m d v^{\prime} / d t^{\prime} .
$$

Thus, we are assured that when $F=m a$ in $\mathrm{W}, F^{\prime}=m a^{\prime}$ in $\mathrm{W}^{\prime}$, where in general $a=d v / d t$. Because of the general relation $F=m a$, we are assured that $F=F^{\prime}$. Newton's second law is invariant under linear displacement of the physical system by changing the frame of reference from which the system is considered. $^{3}$

\section{Conservation of Linear Momentum}

Also, as $p=m v(11)$ and $v_{x_{2}}=v_{x_{2}^{\prime}}{ }_{2}, m d v_{x_{2}}=m d v_{x_{2}^{\prime}}$. For a physical system that initially is at rest in $\mathrm{W}$, whether the system is at $x_{2}$ or $x_{2}^{\prime}=x_{2}+\Delta x$ in $\mathrm{W}^{\prime}, p_{x_{2}}=p_{x_{2}^{\prime}}=0$. In applying the force which imparts a velocity to the system in both $\mathrm{W}$ and $\mathrm{W}^{\prime}$, one can integrate over time and find that $m v_{x_{2}}=m v_{x_{2}^{\prime}}$, where $x_{2}^{\prime}=x_{2}+\Delta x$, or equivalently $p_{x_{2}}=p_{x_{2}^{\prime}}$ (12). Eqn. 12 is possible due to the invariance of Newton's second law of motion in that $a=a^{\prime}$ (10). Thus linear momentum is conserved under linear displacement of the physical system by considering the system in $\mathrm{W}$ and $\mathrm{W}^{\prime}$.

Instead of changing the inertial reference frame, a linear displacement of the same magnitude can be accomplished by shifting the physical system itself

\footnotetext{
${ }^{3}$ The motion of a physical system along the $y$ and $y^{\prime}$ axes of $\mathrm{W}$ and $\mathrm{W}^{\prime}$, respectively, is not discussed because the $y$ and $y^{\prime}$ axis do not move relative to one another. Thus, a force applied to a physical system along the $y$ axis in $\mathrm{W}$, for example, will have the same effect on this system as when the force is considered in $\mathbf{W}^{\prime}$ where it is applied to the system along the $y^{\prime}$ axis.
} 


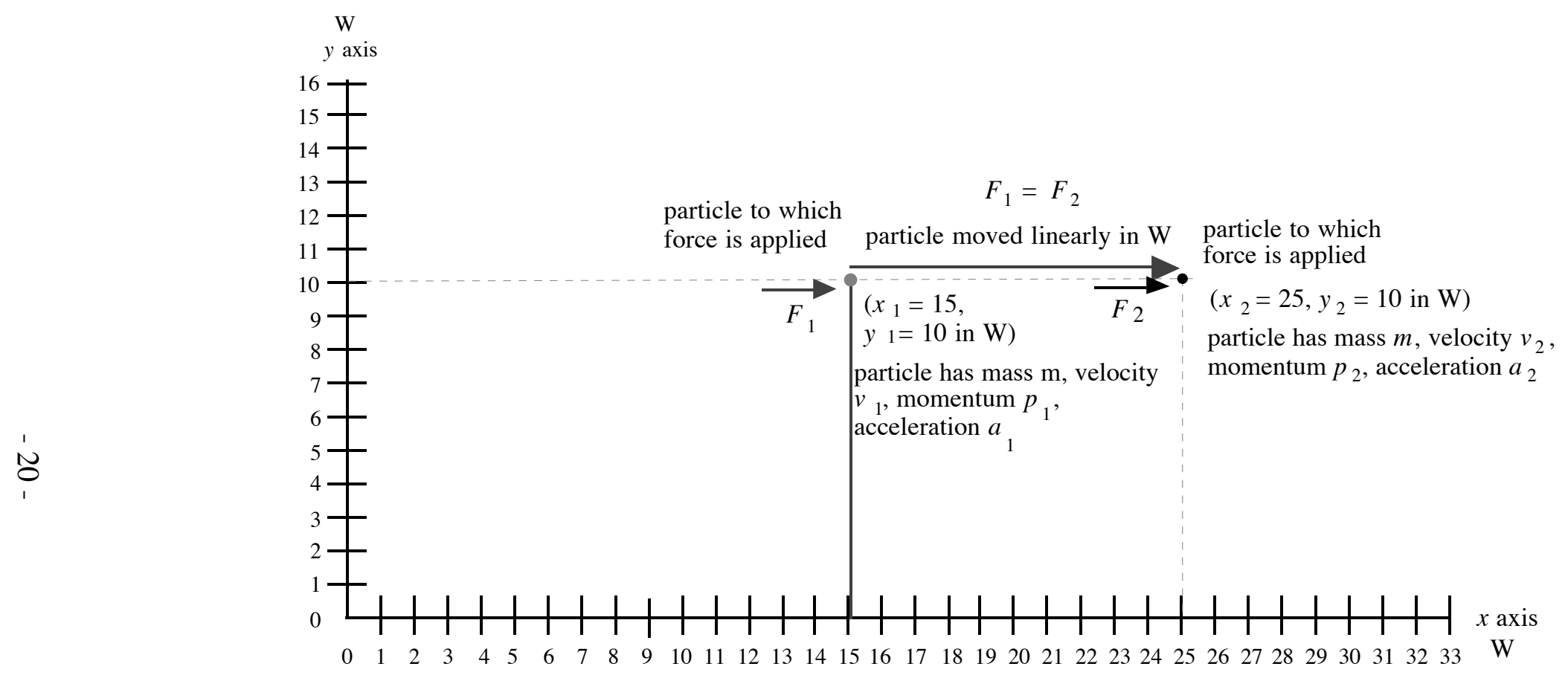

Figure 5

Linear translation of a physical system by shifting the physical system itself linearly with the effect on certain kinematic and dynamic quantities of the system shown. 


\section{The Reference Frame}

linearly relative to $\mathrm{W}$ as depicted in Figure 5. Here, the relationship between the velocity $v_{1}$ of the system at $x_{1}=15$ and the velocity $v_{2}$ of the system at $x_{2}=25$ are the same, that is $v_{1}=v_{2}$. The force $F_{1}$ at $x_{1}$ equals the force $F_{2}$ at $x_{2}$ because the force itself is independent of its location relative to the origins of the space and time axes of $\mathrm{W}$. Thus, when $F_{1}=m a_{1}$ at $x_{1}, F_{2}=m a_{2}$ at $x_{2}$. Newton's second law is invariant under linear displacement of the physical system, whether this displacement is gauged relative to a new inertial reference frame or to a change of position of the system itself in the original inertial reference frame.

Also, as $p=m v$ in general and $v_{1}=v_{2}, m d v_{1}=m d v_{2}$. For a physical system that initially is at rest in $\mathrm{W}$, whether at $x_{1}$ or $x_{2}, p_{1}=p_{2}=0$. In applying the force $F_{1}$ or $F_{2}$ which imparts a velocity to the system, either at $x_{1}$ or $x_{2}$ respectively, one can integrate over time and find that $m v_{1}=m v_{2}$ or $p_{1}=p_{2}$. Thus, linear momentum is conserved under linear displacement of the physical system itself in W.

Hence, the cognitive component of the inertial reference frame and its associated temporal coordinate system allows for the interchangeability of a mathematical translation between inertial reference frames used in the description of a physical system and the actual change of the system in space when demonstrating the invariance of Newton's second law of motion and the conservation of linear momentum that depends on this invariance. Similar arguments can also be made for the invariance of physical law associated with the conservation laws of energy and angular momentum.

\section{Experimental Support}

Experimental evidence from psychology supports the thesis that the mathematical transformation of a physical system is interchangeable with its physical transformation. This evidence is reported, for example, by Cooper \& Shepard (1984) and Pellizzer and Georgopoulos (1993). In an experiment by Cooper (1975), for example, human subjects were presented with twodimensional plane figures. After a figure was presented, subjects were shown another two-dimensional plane figure (either the same figure or its mirror image) in a rotated orientation relative to the position of the first figure. The subject's task was to determine whether the figure in the latter presentation was the original figure or its mirror-image. It was found that the time taken by subjects to determine that the figure in the second presentation was identical to that in the first presentation was linearly dependent on the degree of rotation of 


\section{Chapter 2}

the figure. In those circumstances where the figure in the second presentation was the mirror-image figure, the linear dependence was preserved but a constant time factor was added, apparently needed to identify the mirror-image figure.

Cooper's results supported earlier results obtained by Shepard and Metzler (1971) involving both two-dimensional plane figures and threedimensional solid figures presented in two dimensions. In another experiment (Cooper, 1975), subjects were asked to first mentally rotate a two-dimensional figure into a certain position. Once a subject indicated the rotation was complete, subjects were then presented with either that figure or its mirror image. The subject's task was to determine whether the figure was the one the subject had been asked to rotate or its mirror image. Whatever the angle of the rotation, it was found that subjects rapidly made this determination. The average time for determining which figure was presented was less than one-half second.

In another experiment (Cooper, 1976), subjects were asked to engage in a mental rotation of a two dimensional figure over a certain angle. The subjects were then interrupted in their process of mental rotation by the presentation of either the figure they were asked to rotate or its mirror image. Sometimes, the orientation of the figure presented matched the expected orientation resulting from the ongoing mental rotation at the time of interruption. In this case, subjects' times to determine whether or not the presented figure was the original one they had been asked to rotate or its mirror image were found to be basically constant at one-half second. When the orientation did not match the ongoing mental rotation, the time to determine whether the figure presented was the same or its mirror image increased in a linear fashion as the angle of discrepancy between the expected orientation resulting from the ongoing mental rotation and the orientation of the newly presented figure increased.

Another experiment indicated that the results obtained in the series of experiments discussed on mental rotation of figures were not tied specifically to the use of a figure and its reflection. Basically, Cooper and Podgorny (1976) found that substituting figures with subtle, local changes from the original figure, instead of using its mirror image, did not affect the amount of time subjects took to determine whether a figure presented after subjects had mentally rotated a particular figure was or was not that figure, whatever the angle of rotation. Other evidence indicates that the neurophysiological representation of an intended movement in a particular direction continuously 


\section{The Reference Frame}

transforms in a rotational manner as the direction of the mental representation of the intended movement changes in a rotational manner (Georgopoulos, Lurito, Petrides, Schwartz \& Massey, 1989; Lurito, Georgakopoulos \& Georgopoulos, 1991; Pellizzer \& Georgopoulos, 1993).

Overall, this evidence from psychology supports the thesis that in cognition a physical system may be mentally rotated to accomplish certain cognitive tasks and that this mental rotation is analogous to the actual rotation of the physical system in the world where the magnitude of its angular velocity is constant.

\section{The Next Step}

Already, an exploration of the nature of the reference frame in Newtonian theory has provided indications that mind is linked to the physical world. The flexibility in the choice of a reference frame and its associated temporal coordinate system, and the dependence of a reference frame on the individual's experiencing himself at rest in that frame, are key elements that allowed for the development of the special and general theories of relativity. These theories will now be explored, and the particular ways in which they demonstrate a direct link between cognition and the physical world will be delineated. Flexibility in the choice of a reference frame and an individual's experiencing himself at rest in that frame will be shown to be at the core of this link. 


\section{Chapter 3}

\section{The Special Theory of Relativity}

A consequence in the laws of electromagnetism which were finally set by Maxwell during the 1860 s and early 1870 s that appeared curious to physicists is that light is identified as electromagnetic waves having a constant velocity in a vacuum. ${ }^{4}$ Light thus appeared to have the same velocity regardless of the inertial reference frame in which this velocity was determined. This invariant velocity of light appeared to conflict with the Galilean principle of relativity that Newton's laws of motion hold in inertial reference frames in uniform translational motion relative to one another.

As has been shown specifically for Newton's second law of motion, the Galilean principle of relativity is based on the Galilean coordinate transformation between two inertial reference frames in uniform translational motion relative to one another. Recall that for a frame of reference with one spatial dimension, this transformation may be represented as

$$
t^{\prime}=t
$$

and

$$
x^{\prime}=x-v t
$$

or

$$
x=x^{\prime}+v t,
$$

where $x$ and $t$ represent spatial and temporal coordinates in one inertial reference frame and $x^{\prime}$ and $t^{\prime}$ represent spatial and temporal coordinates in the other inertial reference frame in uniform translational motion relative to the former. If this coordinate transformation is applied to the motion of light, one would expect the velocity of light to vary in different inertial reference frames in a form prescribed by the transformation. Experiments carried out in the latter 1800s indicated that the velocity of light was indeed independent of the inertial frame of reference from which its velocity was determined (Shankland, 1964). These

\footnotetext{
${ }^{4}$ Maxwell's deduction identifying light with electromagnetic waves appeared in 1865 . After noting the similarity between the velocity of light in air or vacuum deduced in electromagnetic theory and experiment concerning this theory, and other empirical results on the velocity of light obtained without reference to electromagnetic theory, Maxwell (1865) concluded:

The agreement of the results [from two sources noted by Maxwell, one concerning electromagnetism and the other concerning light] seems to show that light and magnetism are affections of the same substance, and that light is an electromagnetic disturbance propagated through the field according to electromagnetic laws. [p. 499]
} 


\section{Chapter 3}

results cast doubt on the existence of a stationary medium (called the ether) through which the electromagnetic waves were presumed to travel (Einstein, 1910/1993).

Nonetheless, even with these developments concerning light, physicists in the late 1800s and early 1900s were reluctant to give up the notion that there was some medium through which light traveled in vacuum. And they were reluctant to admit that the Galilean coordinate transformation might not underlie the thesis that the laws of physics hold in inertial reference frames in uniform translational motion relative to one another. Various explanations were attempted to account for the empirical evidence concerning the invariant velocity of light while preserving the Galilean principle of relativity and the Galilean coordinate transformation.

In 1905, Einstein (1905/1952) proposed that: (1) there was no ether through which electromagnetic radiation traveled, and (2) rather than considering the invariant velocity of light to be in conflict with the relativity principle concerning Newton's laws, one should consider the invariant velocity of light to be one manifestation of a broader relativity principle in which the laws of physics hold in inertial reference frames in uniform translational motion relative to one another. On the basis of (2), which entailed the acceptance without reservation of the invariant velocity of light in inertial reference frames in uniform translational motion relative to one another, Einstein was able to develop the theory of special relativity.

Einstein realized, though, that in accepting (2) he was fundamentally altering the Newtonian conception of time as expressed in the Galilean transformation equation $t^{\prime}=t$. He realized that the time of physical events in an inertial reference frame depends on having a valid notion of simultaneity and that, in his new theory, simultaneity depended on the invariant and finite velocity of light. When light was used to establish simultaneity in an inertial reference frame (which means establishing the synchronization of clocks at rest in this reference frame at spatial points distant from one another), Einstein found that the simultaneity established in one inertial reference frame was not the same simultaneity that would be established in an inertial reference frame in uniform translational motion relative to the former. He found that the readings of spatially separated clocks that would be considered synchronous in the former inertial reference frame would not be considered synchronous in the latter inertial reference frame. 


\section{The Special Theory}

To measure the length of a rod in an inertial reference frame requires determining the spatial coordinates of the ends of the rod at a particular time; the temporal coordinates associated with the measurement of each of the spatial coordinates must be the same. Suppose the length of a rod is measured in an inertial reference frame in which the rod is at rest. In an inertial reference frame moving in a uniform translational manner relative to the former reference frame, the simultaneity applicable to the former reference frame does not hold. Thus when the rod is measured in the latter inertial frame, a different form of simultaneity is introduced. It cannot be assumed that the rod will have the same length in both inertial reference frames, as it indeed has in a Newtonian framework.

Unlike Newtonian mechanics, what one finds in special relativity is that the rod which is at rest in one inertial reference frame and moving uniformly in a translational manner in the other frame does not have the same length when measured by observers at rest in one or the other of these reference frames. In the reference frame in which the rod is moving, its length is shorter than in the reference frame in which it is at rest. A similar result is reached with regard to the temporal duration of physical events in inertial reference frames. The duration of some physical occurrence (e.g., the half-life of a muon) which does not change its position in an inertial reference frame is shorter than the duration of this occurrence in an inertial reference frame in which the position of the occurrence moves in a uniform translational manner (e.g., the muon is moving).

The reciprocal nature of the spatial lengths of objects and the duration of occurrences in inertial reference frames in uniform translational motion relative to one another is portrayed in Tables 1 and 2 in Appendix A. In these tables, the dependence of this reciprocity on which inertial reference frame is considered "stationary" and which is considered "moving" in the argument on the relativity of simultaneity is shown.

In special relativity, one very important feature of the space and time of Newton's laws is maintained, and that is their essentially Euclidean nature. Characteristic of this essentially Euclidean nature is that a unit of length is constant throughout any particular inertial reference frame and so is a unit of time. It also should be noted that the term spacetime, rather than space and time, is often used by physicists because of the mutual dependence of space and time in special relativity (Snyder, 1989). 


\section{Chapter 3}

\section{Einstein's 1917 Argument on \\ the Relativity of Simultaneity}

With the previous introduction to the special theory, Einstein's argument on the relativity of simultaneity in inertial reference frames in uniform translational motion relative to one another will now be discussed in some detail. It is the relativity of simultaneity that is responsible for the results obtained in the special theory that are different than those found in Newtonian mechanics in which simultaneity is absolute (i.e., $t^{\prime}=t$ ). The special theory also accounts for the results obtained in Newtonian mechanics that depend on the Galilean coordinate transformation. Demonstrating that there is a cognitive feature in the relativity of simultaneity indicates that a cognitive component is at the base of other results obtained in the special theory. The relativity of simultaneity will be argued using a slightly modified version of Einstein's 1917 gedankenexperiment (i.e., thought experiment) (Einstein, 1917/1961). The gedankenexperiment involves a railway train that is moving with a uniform translational velocity $v$ along an embankment. Both the train and embankment are considered inertial frames of reference.

An observer $\left(\mathrm{O}_{\mathrm{t}}\right)$ is located on the railway train midway between the ends of the train. In addition, an observer $\left(\mathrm{O}_{\mathrm{e}}\right)$ is located midway between the points on the embankment corresponding to the ends of the train just when two lightning flashes strike the ends of the train. Let the motion of the train and the light flash in event $\mathrm{A}$ have the same direction in the reference frame of the embankment, and the motion of the train and the light flash in event B have opposite directions in the reference frame of the embankment.

Einstein wrote concerning the special theory that two events, which each give off a flash of light, may be considered simultaneous in an inertial frame of reference when an observer located midway between the spatial locations of the events observes these flashes of light emitted in both events at the same time (Einstein, 1917/1961). According to Einstein's argument, due to the postulated and empirically validated invariant velocity of light, the two lightning flashes in events $\mathrm{A}$ and $\mathrm{B}$ meet at the observer $\mathrm{O}_{e}$ who is located at the midpoint of the embankment. The events $\mathrm{A}$ and $\mathrm{B}$ in which these flashes occurred are considered simultaneous in the reference frame of the embankment, in accordance with Einstein's definition. (Please see Figure 6.) Here, the embankment is the "stationary" inertial reference frame, the reference frame where the argument on the relativity of simultaneity begins, where simultaneity 


\section{The Special Theory}

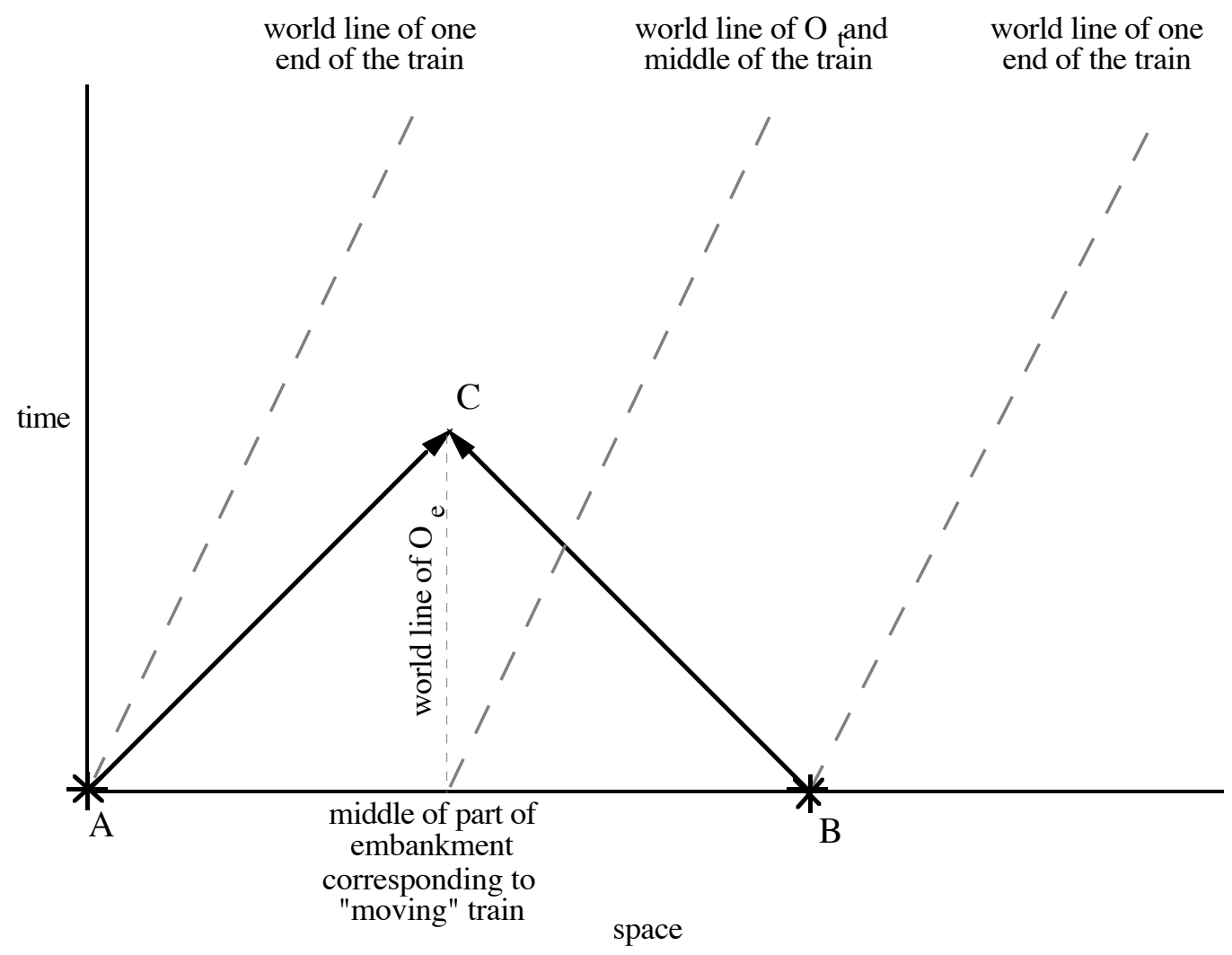

Time and space axes for the inertial reference frame of the embankment.

* are the events at which light flashes are emitted from the postions of the embankment corresponding to the ends of the train with the frame of the embankment considered the "stationary" reference frame.

Figure 6

Simultaneity in the inertial reference frame of the embankment according to Einstein's train gedankenexperiment.

is first established and time first delineated for an inertial reference frame. Einstein described this scenario in the following way:

When we say that the lightning strokes $A$ and $B$ are simultaneous with respect to the embankment, we mean: the rays of light emitted at the places $\mathrm{A}$ and $\mathrm{B}$, where the lightning occurs, meet each other at the mid-point $M$ of the length $A-->B$ of the embankment. But the events $\mathrm{A}$ and $\mathrm{B}$ also correspond to 


\section{Chapter 3}

positions $\mathrm{A}$ and $\mathrm{B}$ on the train. Let $\mathrm{M}^{\prime}$ be the mid-point of the distance A --> B on the travelling train. Just when the flashes of lightning occur [that result in the flashes of light noted above], this point $\mathrm{M}^{\prime}$ [the midpoint of the train] naturally coincides with the point $\mathrm{M}$ [the midpoint of that part of the embankment corresponding to the "moving" train], but it moves...with the velocity $v$ of the train. (Einstein, 1917/1961, pp. 25-26)

To which reference frame are the flashes of lightning first referred to? In which reference frame are they used in an attempt to establish simultaneity, and thus also time, first in accordance with Einstein's definition? Einstein answered the question in writing that "the rays of light at the places $\mathrm{A}$ and B...meet each other at the mid-point $M$ of the length $A-->B$ of the embankment." He also answered these questions when to the phrase, "Just when the flashes of lightning occur," he added the footnote:

${ }^{1}$ As judged from the embankment. (Einstein, 1917/1961, p. 26)

It is the time in the reference frame of the embankment which is given priority and established first in Einstein's argument on the relativity of simultaneity.

But, Einstein argued, the situation for the observer $\mathrm{O}_{\mathrm{t}}$ on the train is different. Einstein wrote:

Now in reality (considered with reference to the railway embankment) he [the observer on the train] is hastening [italics added] towards the beam of light coming from B, whilst he is riding on ahead [italics added] of the beam of light coming from A. Hence the observer will see the beam of light emitted from B earlier than he will see that emitted from A. Observers who take the railway train as their reference-body must therefore come to the conclusion that the lightning flash B took place earlier than the lightning flash A. (Einstein, 1917/1961, p. 26)

Where the reference frame of the embankment is the "stationary" reference frame, for $\mathrm{O}_{\mathrm{t}}$, the lightning flashes have different effective velocities, depending on whether the particular beam is moving in the same or opposite direction to that of the train. (The measured velocity of light by $\mathrm{O}_{t}$ is, of course, the same finite and invariant value it has in all inertial reference frames. It is this finite and invariant value that is central to Einstein's criterion for simultaneity in an inertial reference frame.) In the case where the train has the uniform velocity 


\section{The Special Theory}

$v$ and the lightning flashes have the invariant velocity $c$ for $\mathrm{O}_{\mathrm{e}}$ on the embankment, the light flash from A has the effective velocity $c-v$ and the light flash from $\mathrm{B}$ has the effective velocity $c+v$ relative to $\mathrm{O}_{\mathrm{t}}$ on the train. (Please see Appendix B for further discussion of effective velocities of light.)

In Figure 7, it can be seen that the flashes of light that meet $\mathrm{O}_{e}$ at $\mathrm{C}$ do not meet at the observer $\mathrm{O}_{t}$ who is at rest midway between the ends of the train. When $\mathrm{O}_{t}$ applies Einstein's criterion for simultaneity for an inertial reference frame to these light flashes, he finds the criterion is not met. Thus, Einstein concluded that two occurrences which are simultaneous for the observer at rest in the inertial reference frame of the embankment are not simultaneous for the observer at rest in the inertial reference frame of the train. (In that the observer on the train "is hastening towards the beam from light from B, whilst he is riding on ahead of the beam of light coming from A," Einstein confirmed that the observer on the train is using the time of the reference frame of the embankment to determine whether the simultaneity of the reference frame of the embankment holds in the reference frame of the train as well.) $)^{5}$

In Einstein's scenario, the train is the "moving" inertial reference frame, the reference frame where the time of the "stationary" reference frame is applied through the use of the terms $c-v$ and $c+v$ to determine whether Einstein's criterion for simultaneity in the reference frame of the train is met. In terms of the space of the inertial reference frame of the railway embankment, the midpoint of the train will be displaced by $v \Delta t$, where $v$ is the uniform translational velocity of the train relative to the embankment and $\Delta t$ is the time in the reference frame of the embankment taken by the rays of light that strike the ends of the train to reach $\mathrm{O}_{\mathrm{e}}$ at the midpoint of the embankment.

\section{Switching Which Reference Frame Is}

"Stationary" and Which Is "Moving"

Further discussion is needed on this last point concerning the arbitrary nature of deciding which reference frame is "stationary" and which is "moving" for the purpose of arguing the relativity of simultaneity. The argument presented above concerning the relativity of simultaneity may be applied almost

\footnotetext{
${ }^{5}$ It should be noted with regard to the train gedankenexperiment that if the "moving" observer synchronized his own clocks without relying on the synchronization of the clocks in the "stationary" inertial reference frame, the clocks of the "moving" observer would not be subject to the effective velocities $c+v$ or $c-\mathrm{v}$. Instead, the "moving" observer would only consider the light flashes in terms of their finite and invariant velocity $c$.
} 


\section{Chapter 3}

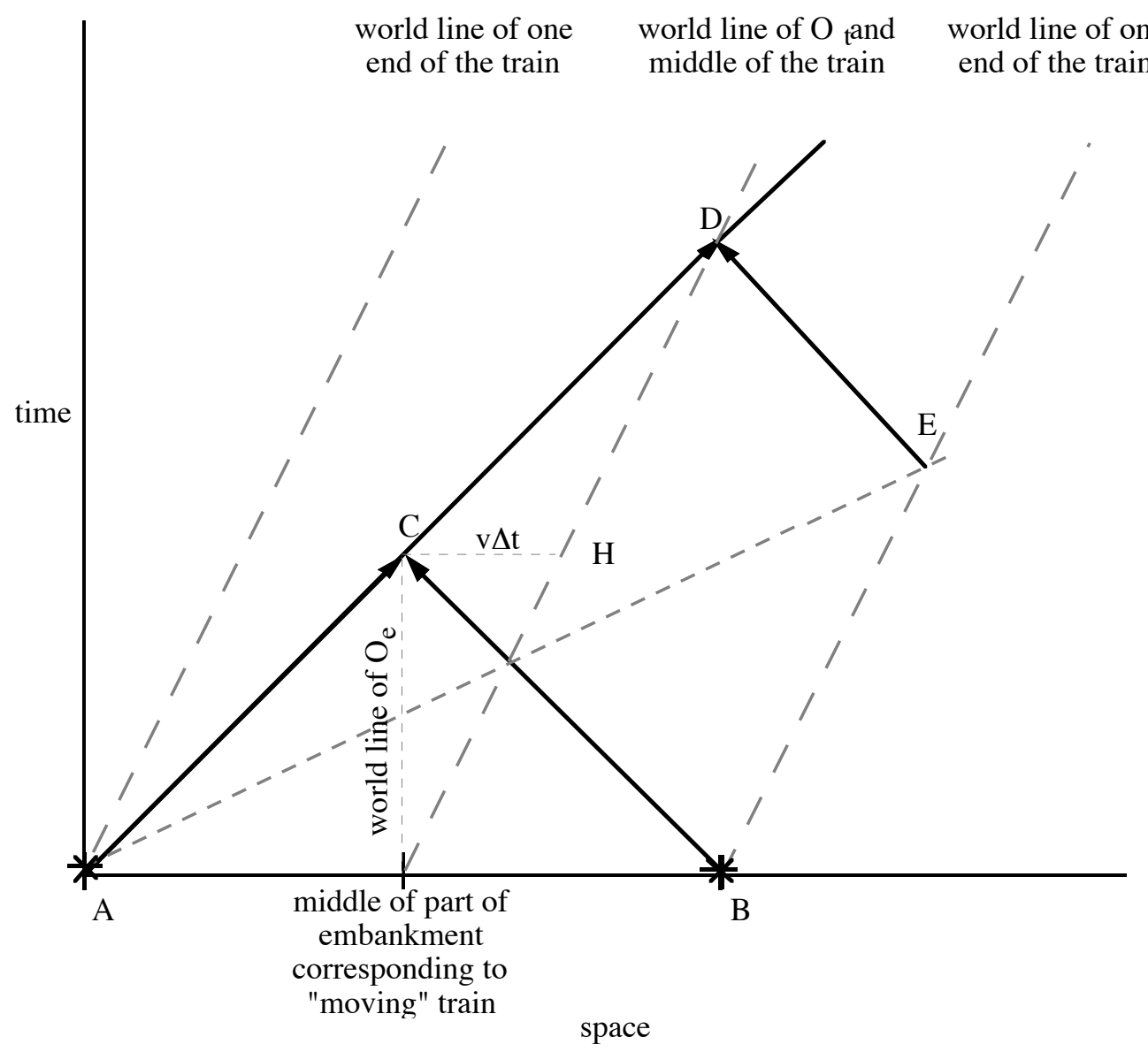

Time and space axes for the inertial reference frame of the embankment.

* are the events at which light flashes are emitted from the postions of the embankment corresponding to the ends of the train with the frame of the embankment considered the "stationary" reference frame.

Figure 7

Simultaneity in the inertial reference frames of the embankment and the train with the embankment considered the "stationary" reference frame and the train the "moving" reference frame.

exactly to the situation in the train gedankenexperiment where $\mathrm{O}_{t}$ at rest on the train is considered at rest in the "stationary" inertial reference frame and $\mathrm{O}_{e}$ at rest on the embankment is considered at rest in the "moving" inertial reference frame. Indeed, the only two changes are:

1) the switch of roles as to which reference frame is the "stationary" reference frame (i.e., in which inertial 


\section{The Special Theory}

reference frame simultaneity is first established) and which is the "moving" reference frame;

2) the reversal in direction of the velocity of the embankment and the train relative to one another.

In the scenario where the train is considered the "stationary" reference frame and the embankment the "moving" reference frame, because of the postulated and empirically validated invariant velocity of light, the two light flashes meet at the observer $\mathrm{O}_{t}$ located midway on the train. The motion of the embankment and the light flash from B have the same direction in the reference frame of the train, and the motion of the embankment and the light flash from A have opposite directions in the reference frame of the train. (Please see Figure 8.)

The lightning flashes are considered simultaneous in the reference frame of the train (in this scenario, the "stationary" reference frame), in accordance with Einstein's definition of simultaneity. As deduced by the "stationary" observer on the train, the light flash in A has the velocity $c+v$ relative to the "moving" observer on the embankment and the light flash in B has the velocity $c-v$ relative to the observer on the embankment. In Figure 8 , it can be seen that the flashes of light that meet $\mathrm{O}_{t}$ at $\mathrm{C}$ do not meet at the observer $\mathrm{O}_{\mathrm{e}}$ who is at rest midway between the ends of the embankment. When $\mathrm{O}_{\mathrm{e}}$ applies Einstein's criterion for simultaneity for an inertial reference frame to these light flashes, he finds the criterion is not met. Similar to the first scenario, it can be concluded that two occurrences which are simultaneous for the observer at rest in the inertial reference frame of the train are not simultaneous for the observer at rest in the inertial reference frame of the embankment.

There is nothing that points in any way to an individual arguing the relativity of simultaneity in one direction or the other, that is with one of the reference frames more likely than the other to be designated the "stationary" reference frame. If one were more likely, the fundamental tenet of the special theory that there is no preferred inertial reference frame as concerns the description of physical phenomena for observers at rest in their respective inertial reference frames in uniform translational motion relative to one another would be violated. There would be a preferred inertial reference frame, and it would be the reference frame designated the "stationary" reference frame. There is thus a free choice on the part of the individual making the argument on the relativity of simultaneity as to which direction the argument should proceed. 


\section{Chapter 3}

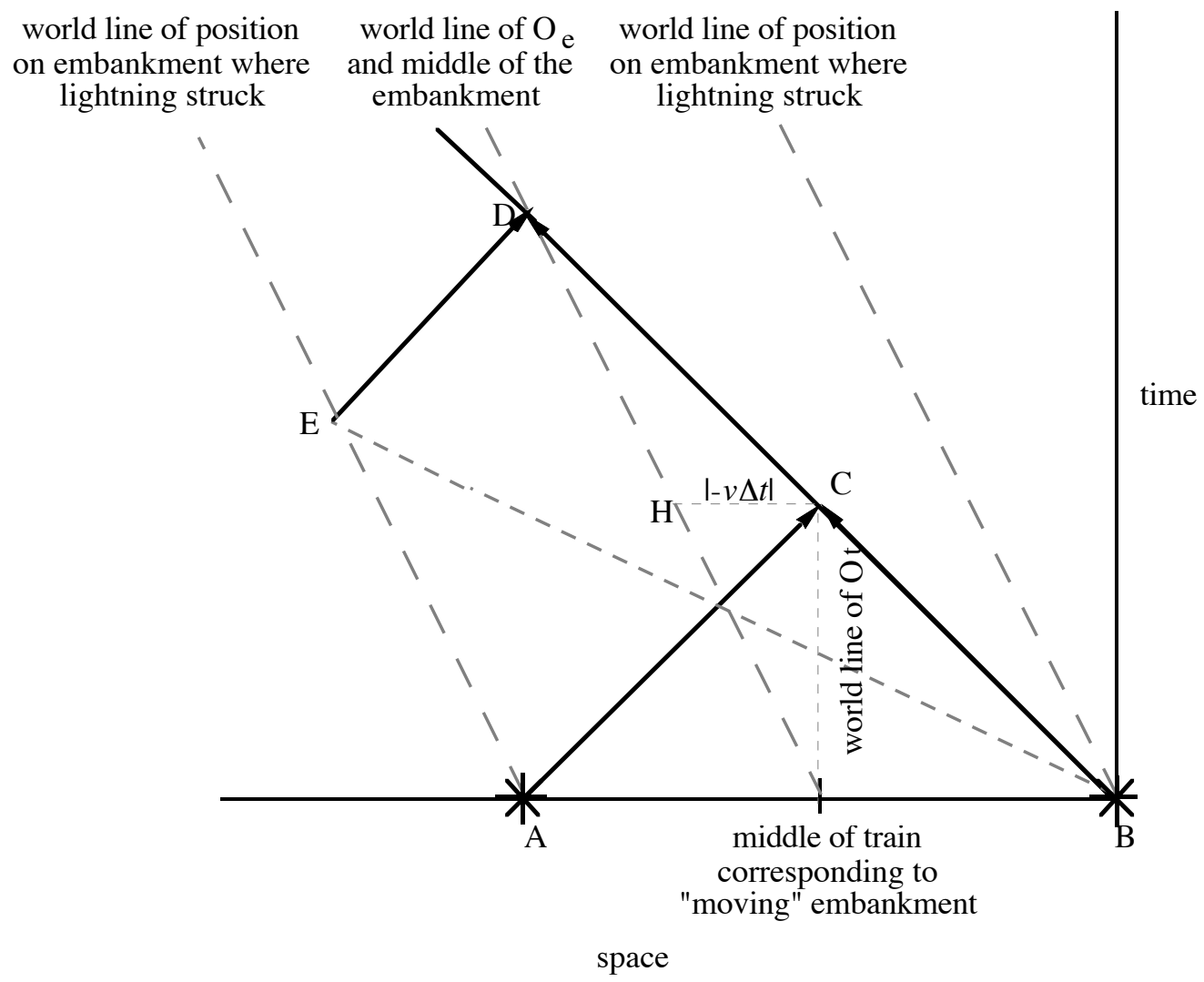

Time and space axes for the inertial reference frame of the train.

* are the events at which light flashes are emitted from the ends of the train corresponding to positions on the embankment with the frame of the train considered the "stationary" reference frame.

Figure 8

Simultaneity in the inertial reference frames of the embankment and the train with the train considered the "stationary" reference frame and the embankment the "moving" reference frame.

In that this choice is not limited in any way by a physical factor in the special theory, it can be said that the choice is fundamentally a cognitive one, not reducible to some physical phenomenon, and that this cognitive choice is reflected in distinct sets of empirical results found when certain measurements are taken in the physical world, some of which are delineated in Appendix A. Whichever direction is chosen, this direction will correspond to a distinct set of results obtained by an observer at rest in his inertial reference frame and which he considers the "stationary" reference frame. It should be noted that the 


\section{The Special Theory}

assumption, supported empirically, that an observer at rest in his inertial reference frame considers this reference frame "stationary" and the other inertial reference frame "moving" supports the premise that there is no cognitive factor that prompts an individual to argue the relativity of simultaneity in one direction or the other.

The Arbitrary Decision in the

Argument on the Relativity

of Simultaneity and Some Consequences

The basic point is this. If the railway embankment is considered the "stationary" reference frame, then in Einstein's argument simultaneity is first established in this inertial reference frame in terms of the motion of light. The light flashes leaving from the points of the embankment corresponding to the ends of the train meet midway on the embankment between these two points. Concerning the train, these light flashes certainly do not meet midway on the train. This is seen in an inspection of Figure 6. As noted, simultaneity in the reference frame of the embankment is defined as the meeting at $\mathrm{C}$ of the light flashes represented by AC and BC. As can be seen in Figure 7, simultaneity in the train, the "moving" reference frame, is achieved when the flash leaving from the end of the train in event A meets at event $\mathrm{D}$ what appears to be a new flash leaving from the other end of the train in event $\mathrm{E}$. $\mathrm{E}$ is an event on the $x^{\prime}$ axis. That the event $\mathrm{D}$ represents the light flashes $\mathrm{AD}$ and $\mathrm{ED}$ meeting midpoint on the train is evident from an inspection of Figure 9, which essentially is Figure 7 on which coordinate grids for the inertial reference frames of the embankment and the train have been added. (In Figure 9, the inertial reference frame of the embankment is also referred to as $\mathrm{W}$, and the inertial reference frame of the train is referred to as $\mathrm{W}^{\prime}$.)

The Spatial Length Along the Axis of Motion

of Physical Existents at Rest in Either the

"Stationary" or "Moving" Reference Frame

One can see in Figure 9 that the length of the train measured by observers at rest on the train is not 10 units but more than 10 units, specifically $10 /\left(1-\mathrm{v}^{2} / \mathrm{c}^{2}\right)^{1 / 2}$ units, because the light flashes $\mathrm{AD}$ and $\mathrm{DE}$ meet Einstein's criterion for simultaneity that light flashes from the ends of the train meet the observer at rest located midpoint on the train, when the train is considered the "moving" inertial reference frame. When the train is the "moving" reference frame, the events A and D correspond to the ends of the train at the same time in 


\section{Chapter 3}

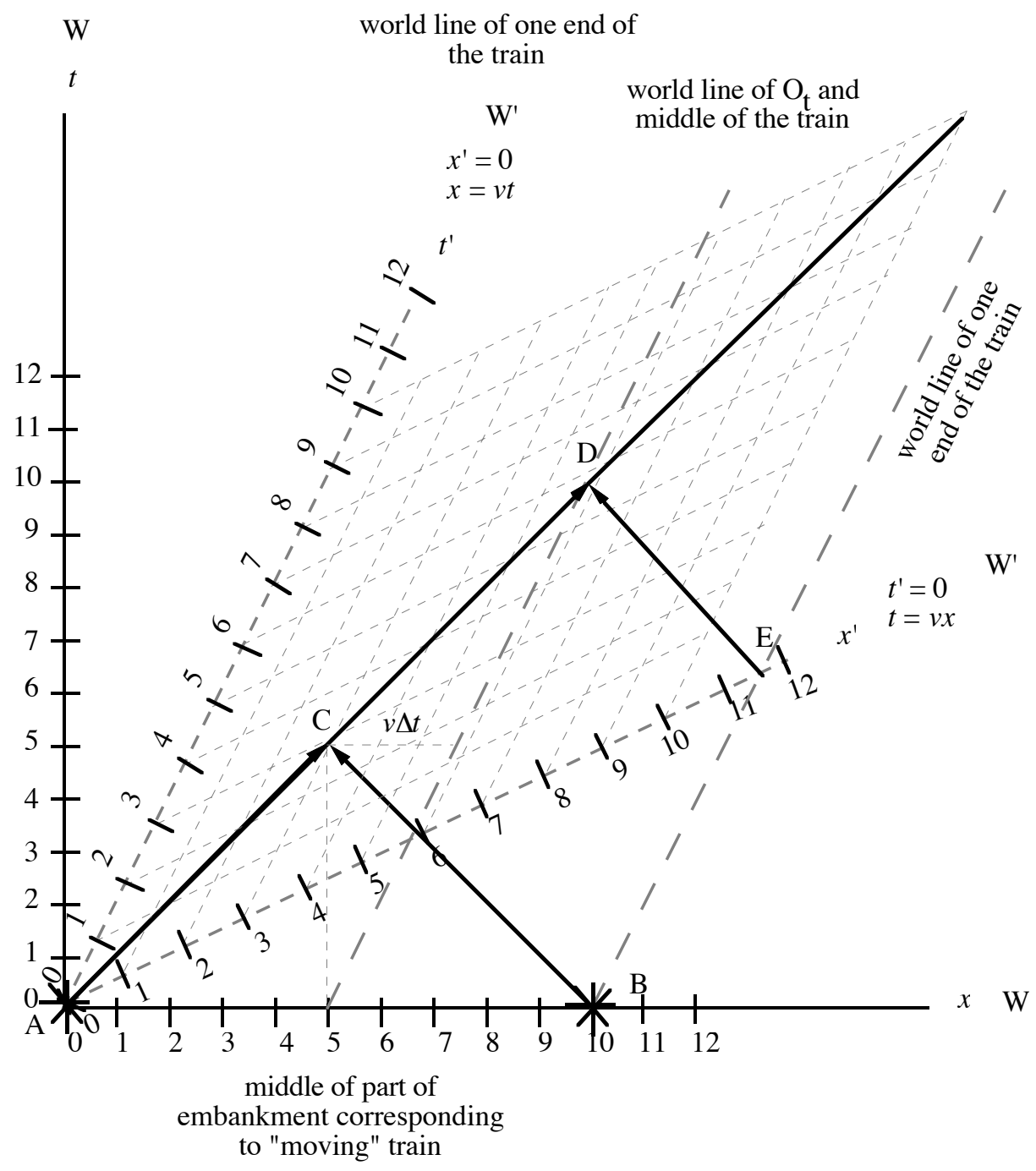

\footnotetext{
$\Psi$ are the events at which light flashes are emitted from the postions of the embankment corresponding to the ends of the train with the frame of the embankment considered the "stationary" reference frame.
}

\section{Figure 9}

Minkowski diagram of $\mathrm{W}$ and $\mathrm{W}^{\prime}$ showing simultaneity in the inertial reference frames of the embankment $(\mathrm{W})$ and the train $\left(\mathrm{W}^{\prime}\right)$ with the embankment considered the "stationary" frame.

the reference frame of the train because the light flashes $\mathrm{AD}$ and $\mathrm{DE}$ meet Einstein's criterion for simultaneity. Since A and D correspond to the ends of the train at the same time, the length from A to D is a measurement of the length 


\section{The Special Theory}

of the train for observers at rest in the "moving" reference frame of the train. This length, $10 /\left(1-\mathrm{v}^{2} / \mathrm{c}^{2}\right)^{1 / 2}$ units, is established in the argument on the relativity of simultaneity after length is established in the "stationary" reference frame of the embankment where light flashes occur in events A and B that are equidistant from the observer at rest in the "stationary" reference frame of the embankment.

Consider the reverse scenario, where the train is considered the "stationary" reference frame and the embankment is considered the "moving" reference frame. The light flashes are the same physical phenomena as in Einstein's scenario. In the reverse scenario, the light flashes $\mathrm{AH}$ and $\mathrm{GH}$ in Figure 10 are used to establish simultaneity in the inertial reference frame of the train first in the argument on the relativity of simultaneity. The light flashes $\mathrm{AD}$ and DE, found in Figure 9, do not appear to be those flashes which are used to establish simultaneity in the reference frame of the train in Figure 10. One would think that the same light flashes that were used to establish simultaneity in Einstein's scenario would be used in the reverse scenario being discussed. But in comparing Figures 9 and 11 (where the space and time axes for the inertial reference frame of the embankment are drawn in), one can see even more clearly that this does not appear to be the case. (For more information on the nature of these light flashes, please see Appendix C.)

Regardless of the particular world lines of the light flashes, the distance between the flashes in whichever inertial reference frame is designated the "stationary" reference frame needs to be the same. In the reverse scenario, the distance between the light flashes used to establish simultaneity in the "stationary" inertial reference frame of the train is 10 units, the length of the train.

One can see in Figure 11, essentially Figure 10 with coordinates for the reference frame of the embankment, the analogous circumstances to Figure 9 except which reference frame is "stationary" and which is "moving" have been reversed. When the embankment is the "moving" reference frame, the events A and $\mathrm{F}$ are simultaneous in the "moving" reference frame of the embankment because the light flashes AI and FI meet Einstein's criterion for simultaneity. Since A and F correspond to points of the embankment at the same time, the length from $A$ to $F$ is a measurement of the length of a section of the embankment for observers at rest in the "moving" reference frame of the embankment. This length, $10 /\left(1-\mathrm{v}^{2} / \mathrm{c}^{2}\right)^{1 / 2}$ units, is established in the argument on the relativity of simultaneity after length is established in the "stationary" reference frame of the train where light flashes occur in events A and G that are 


\section{Chapter 3}

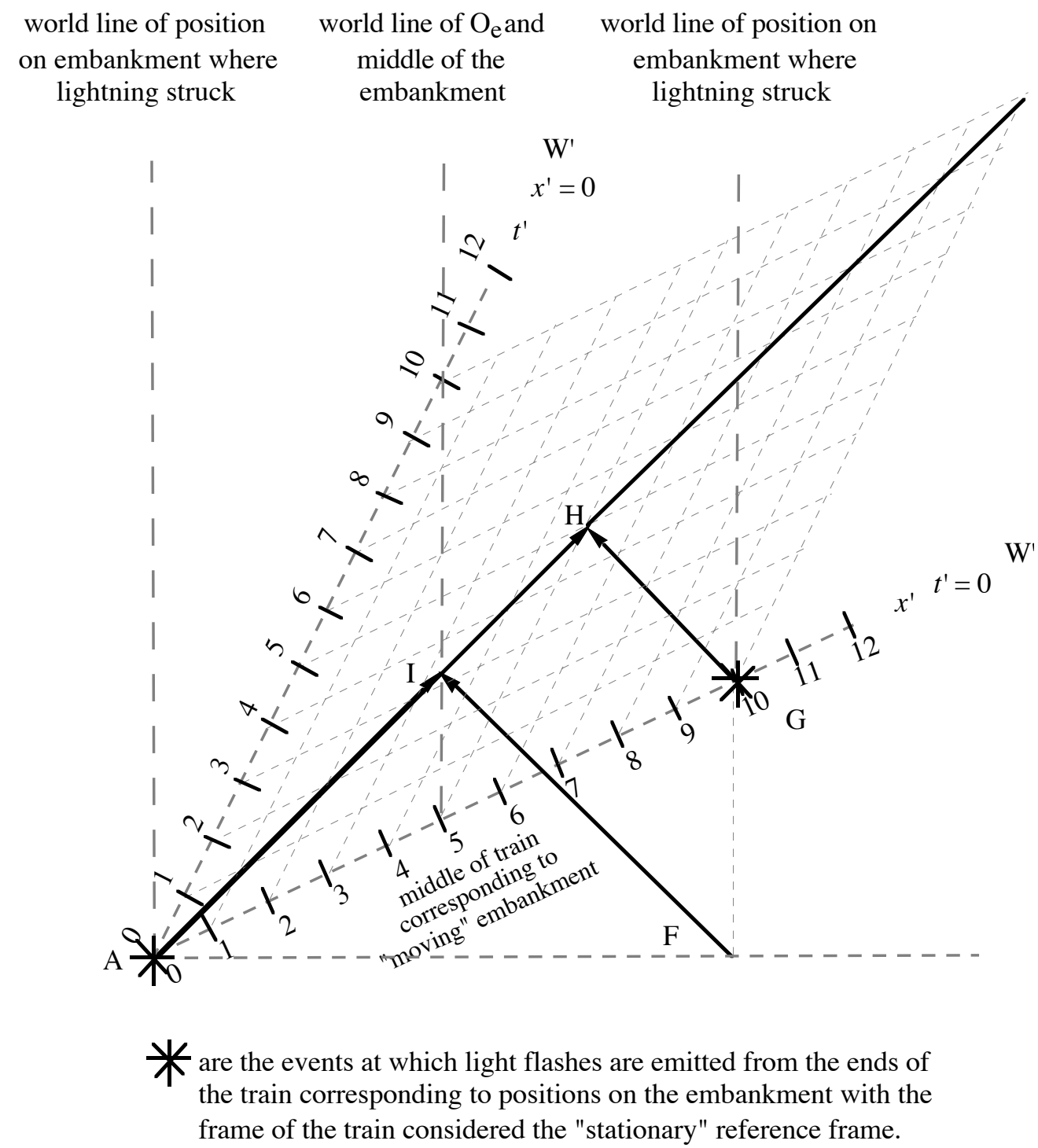

Figure 10

Simultaneity in the inertial reference frames of the train and the embankment with the train considered the "stationary" frame.

equidistant from the observer at rest in the "stationary" reference frame of the train.

This interesting point should be emphasized. The length of an existent at rest in an inertial reference frame as measured by observers at rest in that 


\section{The Special Theory}

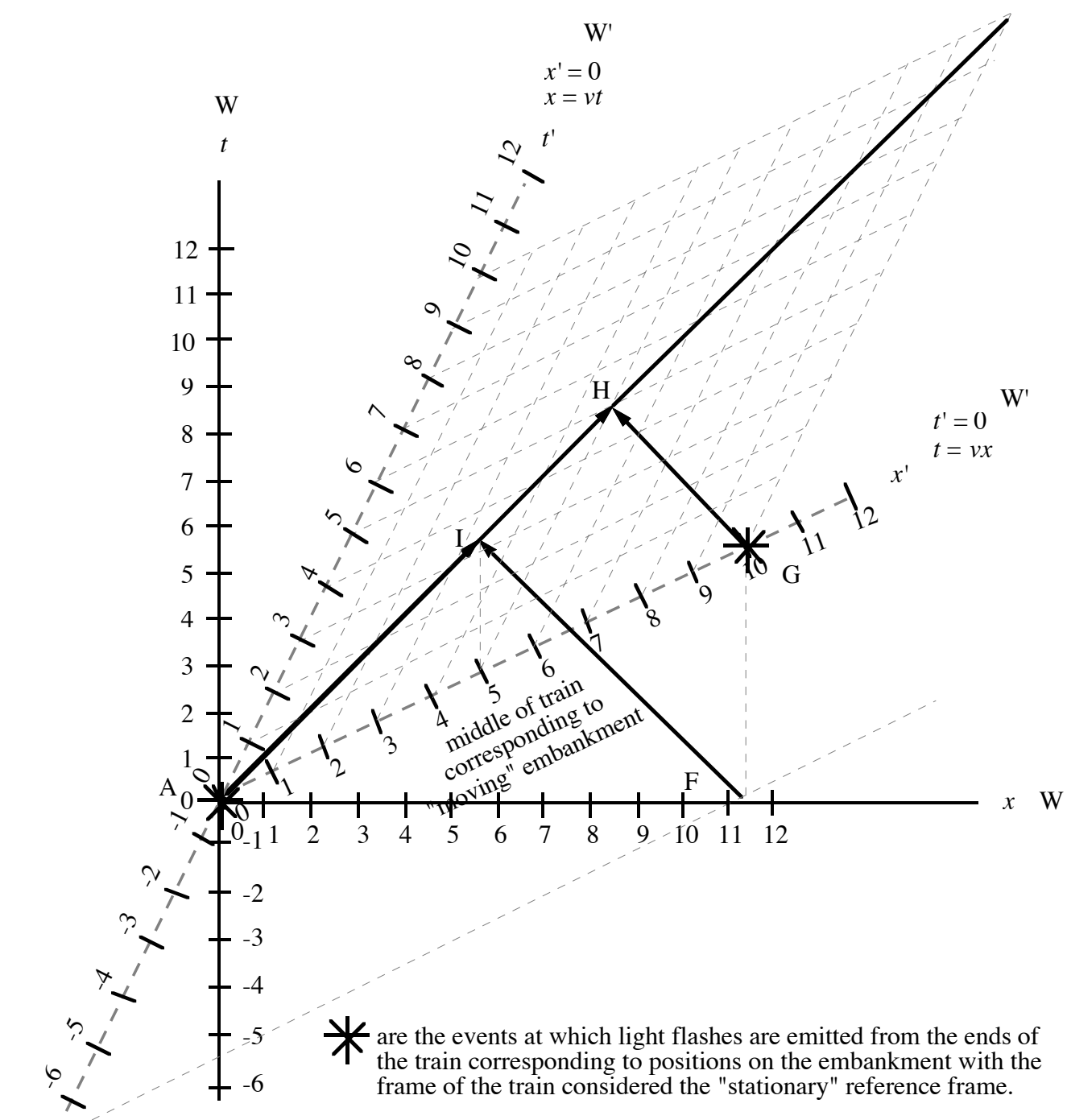

Figure 11

Minkowski diagram of $\mathrm{W}$ and $\mathrm{W}^{\prime}$ showing simultaneity in the inertial reference frames of the train $\left(\mathrm{W}^{\prime}\right)$ and the embankment $(\mathrm{W})$ with the train considered the "stationary" frame.

reference frame is dependent on whether the reference frame is considered the "stationary" or "moving" reference frame in the argument on the relativity of simultaneity. In Figure 10, it can be seen that the light flashes used to delineate simultaneity in the reference frame of the train when it is considered the "stationary" reference frame are from event A to event $\mathrm{H}$ and from event $\mathrm{G}$ to event $\mathrm{H}$. (Event $\mathrm{H}$ is the meeting of the light flashes originating in events $\mathrm{A}$ and 


\section{Chapter 3}

$\mathrm{G}$ midpoint on the train.) Also, the light flashes from event $\mathrm{A}$ to event $\mathrm{C}$ and from event $\mathrm{B}$ to event $\mathrm{C}$ found in Figure 9 do not represent light flashes meeting $\mathrm{O}_{\mathrm{e}}$ midpoint on the embankment when it is the "moving" inertial reference frame. Rather, as seen in Figure 10, the lights flashes from event A to event I and from event $\mathrm{F}$ to event $\mathrm{I}$ represent two light flashes meeting at $\mathrm{O}_{\mathrm{e}}$ midpoint on the embankment when the embankment is the "moving" inertial reference frame.

In terms of the logical sequence in the argument on the relativity of simultaneity, the two light flashes from A to I and from F to I are used to establish simultaneity in the "moving" reference frame of the embankment after simultaneity is first established in the "stationary" reference frame of the train using light flashes from $\mathrm{A}$ to $\mathrm{H}$ and from $\mathrm{G}$ to $\mathrm{H}$. In an inspection of Figure 11 , one can see a bit more precisely that the light flashes striking the ends of the train and the corresponding points on the embankment, and which are used to establish simultaneity in the "stationary" inertial reference frame of the train, do not serve to establish simultaneity in the "moving" inertial reference frame of the embankment. In Figure 11, the $t$ coordinate for $\mathrm{A}$ is 0 , but the $t$ coordinate for $\mathrm{G}$ is close to 5. One also can see from an inspection of Figure 11 that where the $t^{\prime}$ coordinate for $\mathrm{A}$ is 0 , the $t^{\prime}$ coordinate for $\mathrm{F}$ is close to -7 . For $\mathrm{A}$ and $\mathrm{F}$, $t=0$, and $\mathrm{A}$ and $\mathrm{F}$ are simultaneous events in the "moving" inertial reference frame of the embankment.

What has occurred is that the direction in which the argument on the relativity of simultaneity is made has been reversed, and this reversal has changed the nature of space in a particular inertial reference frame such that the length of a concrete measuring instrument at rest in this reference frame and measured by observers at rest in this reference frame is different depending on whether the inertial reference frame in which the instrument is at rest is the "stationary" or the "moving" reference frame in the argument on the relativity of simultaneity. (The fundamental tenet that physical description is equivalent in inertial reference frames in uniform translational motion relative to one another is adjusted to: the description of physical phenomena must be the same for observers at rest in their inertial reference frames when these reference frames are the "stationary" reference frames in the argument on the relativity of simultaneity and the description of physical phenomena must be the same for observers at rest in their inertial reference frames when these reference frames are the "moving" reference frames.) 


\section{The Special Theory}

There is nothing in the physical world that prompts an individual considering inertial reference frames in uniform translational motion relative to one another to choose a particular direction in which to argue the relativity of simultaneity. It should be remembered that the possibility of making the argument in either direction without any limitation by the physical world is central to retaining the central tenet of the special theory that there is no preferred inertial reference frame from which to describe physical phenomena. If there were some reason in the physical world to choose one direction over another, there would be a preferred inertial reference frame from which to describe physical phenomena. The choice in arguing the relativity of simultaneity is an arbitrary one, a free one, on the part of the person considering the relativity of simultaneity. This arbitrary choice by the individual considering the relativity of simultaneity is reflected in observers being at rest in their respective inertial reference frames in uniform translational motion relative to one another and considering the inertial reference frame in which each is at rest the "stationary" reference frame and the other observer's inertial reference frame the "moving" reference frame.

One might think that the different scenarios regarding spatiotemporal relations can be distinguished by the concrete physical existence of the measuring rod in the case of spatial relations or clocks in the case of temporal relations. That is, it might be thought that even though the relativity of simultaneity can be argued in either direction, in practice, only one scenario applies at any one time and this limitation is imposed by the concrete physical world. Essentially, the basis for this thought is that the physical mechanism of a clock or the physical structure of the rod are concrete, that is, they are at rest in only one inertial reference frame and that their basic constitution remains unchanged in whatever inertial reference frame from which they are considered. It has been shown that with regard to measuring rods, it is not the case that their basic constitution remains unchanged.

More generally, the essence of the functioning of a clock in the special theory is the motion of light over a prescribed distance in both directions. It is not the concrete existence of a clock that is at the essence of the functioning of a clock in an inertial reference frame. This is the case because the synchronization of clocks is established in Einstein's 1905 and 1917 definitions of simultaneity (or the synchronization of clocks) by the motion of light over a particular distance in both possible directions (Snyder, 1992b). 


\section{Chapter 3}

This motion of light can be considered periodic motion. Because the functioning of clocks depends on the periodic motion of light traveling over some spatial length and this spatial length itself depends on simultaneity in a particular inertial reference frame, the concrete nature of a clock should not result in its functioning remaining unchanged in different inertial reference frames in uniform translational motion relative to one another. That is, because the length of an existent in an inertial reference frame depends on determining the ends of the existent simultaneously, one would expect that the concrete nature of the existent would not inhibit the fundamental character of the relativity of simultaneity.

\section{The Time of the "Stationary" \\ Inertial Reference Frame}

In the argument on the relativity of simultaneity, the "moving" observer relies on simultaneity and time of the "stationary" reference frame to determine that the criterion for simultaneity is not met by clocks at rest in the "moving" reference frame. The "moving" observer relies on clocks synchronized in the "stationary" reference frame to set clocks at rest in the "moving" reference frame and thus to determine whether the criterion for simultaneity is met in the "moving" reference frame. This has been shown in the train gedankenexperiment and will be shown in Einstein's original argument on the relativity on simultaneity. Einstein explicitly proposed in 1905 in his argument on the relativity of simultaneity that the clocks of the "moving" reference frame are synchronized in accordance with, and rely on, the clocks of the "stationary" reference frame.

In his original paper on the special theory of relativity, Einstein noted that simultaneity (or the common time of clocks) is delineated for an inertial reference frame of reference when, by definition, the time required for a ray of light to travel from a spatial point $\mathrm{A}$ to a spatial point $\mathrm{B}$ is equal to the time required for a ray of light to travel from point B to point A (Einstein 1905/1952). He argued the relativity of simultaneity this way:

We imagine further that at the two ends $\mathrm{A}$ and $\mathrm{B}$ of the rod [moving with uniform translational velocity relative to the stationary inertial system], clocks are placed which synchronize with the clocks of the stationary system, that is to say that their indications correspond at any instant to the "time of the 


\section{The Special Theory}

stationary system" at the places where they happen to be. These clocks are therefore "synchronous in the stationary system."

We imagine further that with each clock there is a moving observer, and that these observers apply to both clocks the criterion established...for the synchronization of two clocks [that the flight time of a light ray in an inertial frame of reference from spatial point $A$ to spatial point $B$ is equal to the flight time of a light ray from point $\mathrm{B}$ to point $\mathrm{A}]$. Let a ray of light depart from A at the time* $t_{\mathrm{A}}$, let it be reflected at $\mathrm{B}$ at the time $t_{\mathrm{B}}$, and reach $\mathrm{A}$ again at the time $t_{\mathrm{A}}^{\prime}$. Taking into consideration the principle of the constancy of the velocity of light we find that

$$
t_{\mathrm{B}}-t_{\mathrm{A}}=\frac{r_{\mathrm{AB}}}{c-v} \quad \text { and } \quad t_{\mathrm{A}}^{\prime}-t_{\mathrm{B}}=\frac{r_{\mathrm{AB}}}{c+\mathrm{v}}
$$

where $r_{A B}$ denotes the length of the moving rod-measured in the stationary system. Observers moving with the rod would thus find that the two clocks were not synchronous, while observers in the stationary system would declare the clocks to be synchronous. * Time here denotes "time of the stationary system" and also "position of hands of the moving clocks situated at the place under discussion." (Einstein 1905/1952, p. 42)

Whether using readings from clocks synchronized in the "stationary" reference frame or the coincidence of flashes of light as judged from the railway embankment, the "moving" observer relies on the time established first in the "stationary" reference frame in the argument on the relativity of simultaneity. This reliance by the "moving" observer on the time established in the "stationary" reference frame sets up a different basis for the development of simultaneity, and thus time, in his own reference frame than would otherwise have been the case if the "moving" observer had instead been the observer at rest in the "stationary" reference frame in the argument on the relativity of simultaneity.

\section{The Rate of Clocks at Rest in Either}

the "Stationary" or "Moving" Reference Frame

It has been shown that the spatial length of a physical existent at rest in an inertial reference frame and aligned along the axis of uniform translational 


\section{Chapter 3}

motion of two inertial reference frames relative to one another depends on whether the reference frame in which the existent is at rest is designated the "stationary" or "moving" reference frame in the argument on the relativity of simultaneity. A similar dependency holds for the rate of a clock in an inertial reference frame. The rate of a clock at rest in an inertial reference frame depends on whether the reference frame in which the clock is at rest is designated the "stationary" or "moving" reference frame in the argument on the relativity of simultaneity.

In Figures 12 and 13, conditions for temporal durations in inertial reference frames in uniform translational motion relative to one another analogous to those used to explore spatial length are presented. In Figures 12 and 13, Einstein's (1905/1952) original argument on the relativity of simultaneity is depicted in its essential elements. (In Figure 12, W is Einstein's "stationary system" [p. 42] and $\mathrm{W}^{\prime}$ is the system with the "moving" rod.) In Figure 12, allow that a distance of $\Delta x=5$ units is used to establish simultaneity in accord with Einstein's original criterion. If a light flash travels from event A to event $\mathrm{B}$, traveling $\Delta x$, and if the light flash is reflected back without delay, it requires the same amount of time for the light flash to return to $x=0$ from $x=5$ (in event C) as it took for the light flash to travel from event A to event B (i.e., 5 units of time). Einstein's original criterion for simultaneity in an inertial reference frame is met and time is established in $\mathrm{W}$, the "stationary" reference frame.

It can be seen in Figure 12 that the simultaneity established in this process in $\mathrm{W}$ is not the same as the simultaneity established in ' $\mathrm{W}^{\prime}$. An inspection of this figure indicates that simultaneity in $\mathrm{W}^{\prime}$ requires another process. Allow that a rod is at rest in $\mathrm{W}^{\prime}$ and thus moving in a uniform translational manner relative to $\mathrm{W}$ and that its length as measured in $\mathrm{W}$ is 5 units. Allow that in line with Einstein's criterion, a light flash begins in event A at one end of the rod, travels to event D where it reaches the other end of the rod, and is immediately reflected back. (Event D occurs on the world line of the end of the rod toward which the light flash travels after first being emitted.) The light flash returns to the end of the rod that it originated from in event $\mathrm{E}$ at $x^{\prime}=0$. In terms of the time of $\mathrm{W}, t_{\mathrm{AD}} \neq t_{\mathrm{DE}}$ and thus Einstein's original criterion for establishing simultaneity is not met in $\mathrm{W}^{\prime}$ when this process is considered in terms of the time of $\mathrm{W}$. From Figure 12, it can also be seen that $t_{\mathrm{AD}}^{\prime} \approx t_{\mathrm{DE}}^{\prime} \approx 6$ units and that simultaneity, and thus time, is established in $\mathrm{W}^{\prime}$ in accordance with Einstein's original criterion for simultaneity in an inertial reference frame. 


\section{The Special Theory}

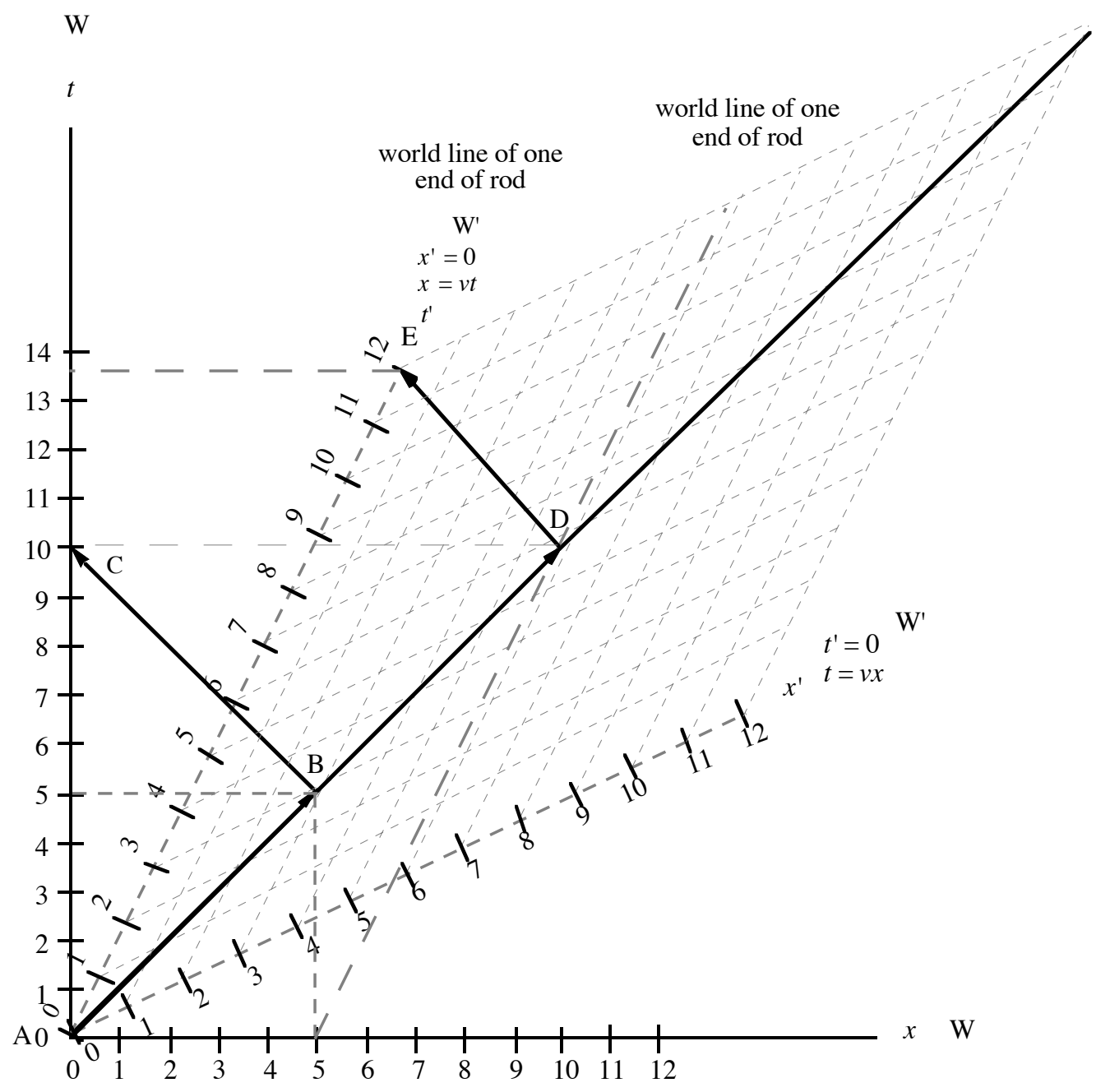

Figure 12

Minkowski diagram of inertial reference frames $\mathrm{W}$ and $\mathrm{W}^{\prime}$ showing simultaneity in accordance with Einstein's 1905 formulation with W the "stationary" reference frame and $\mathrm{W}^{\prime}$ the "moving" reference frame.

In terms of the time of $\mathrm{W}^{\prime}, t^{\prime}$, the passage of the light ray from one end of the rod to the other and back again occurs in about 12 units. If the concern is with the amount of time that elapses in W (the "stationary" reference frame) corresponding to these 12 units in $\mathrm{W}^{\prime}$ (the "moving" reference frame), the 


\section{Chapter 3}

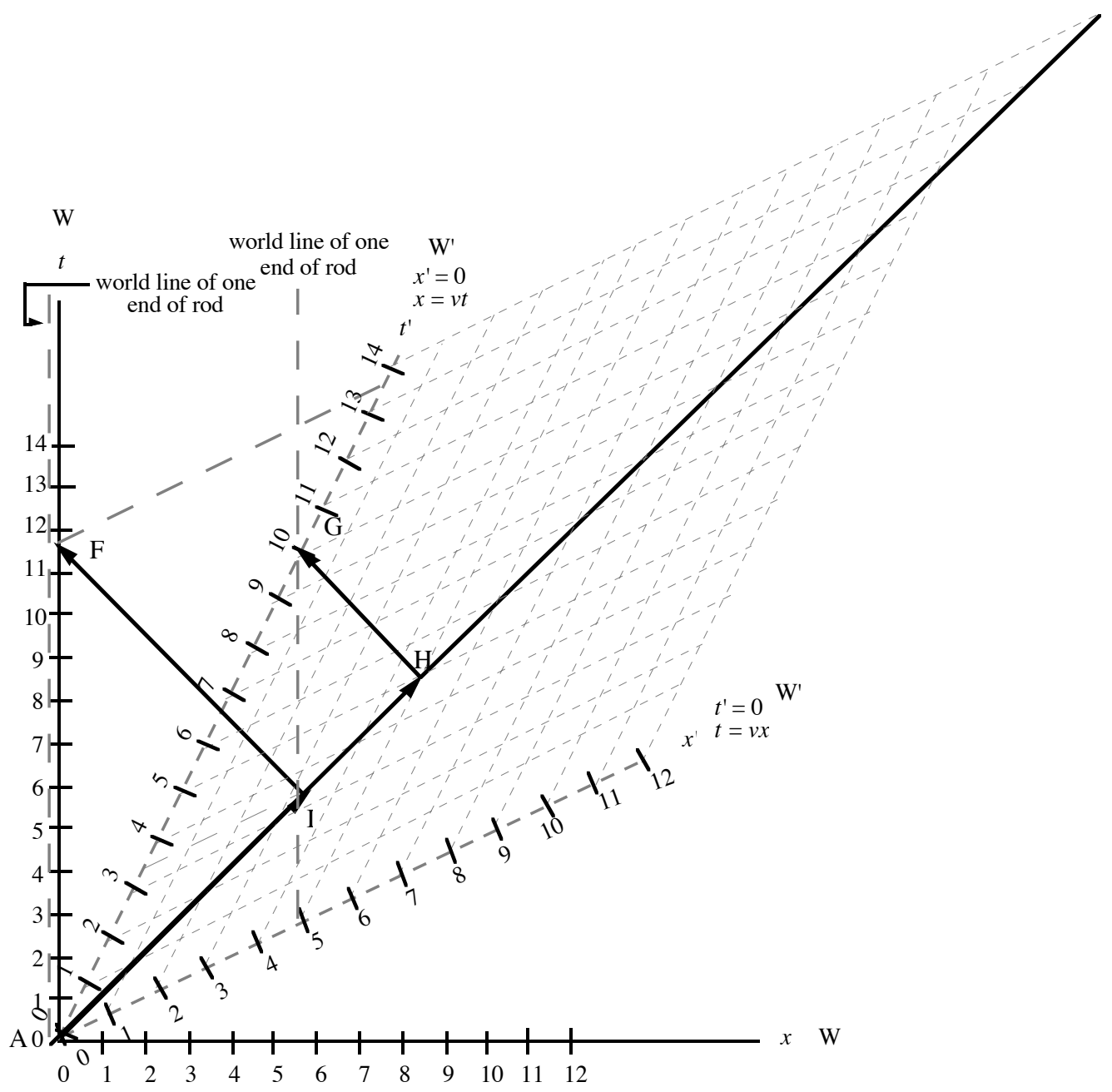

Figure 13

Minkowski diagram of inertial reference frames $\mathrm{W}$ and $\mathrm{W}^{\prime}$ showing simultaneity in accordance with Einstein's 1905 formulation with $\mathrm{W}^{\prime}$ the "stationary" reference frame and $\mathrm{W}$ the "moving" reference frame.

horizontal dashed line parallel to the space axis for $\mathrm{W}$ indicates that the corresponding time in $\mathrm{W}$ is between 13 and 14 units.

Figure 13 displays the reverse scenario where $W^{\prime}$ is the "stationary" inertial reference frame and $\mathrm{W}$ is the "moving" inertial reference frame. Allow that a distance of $\Delta x^{\prime}=5$ units is used to establish simultaneity in accord with Einstein's original criterion. If a light flash travels from event A to event $\mathrm{H}$, that covers $\Delta x^{\prime}$, and if the light flash were reflected back without delay, it 


\section{The Special Theory}

requires the same amount of time for the light flash to return to $x^{\prime}=0$ from $x^{\prime}=5$ (in event G) as it took for the light flash to travel from event A to event $\mathrm{H}$. Einstein's original criterion for simultaneity in an inertial reference frame is met and time is established in W', the "stationary" reference frame.

It can be seen in Figure 13 that the simultaneity established in this process in $\mathrm{W}^{\prime}$ is not the same as the simultaneity established in $\mathrm{W}$. An inspection of this figure indicates that simultaneity in $\mathrm{W}$ requires another process. Allow that a rod is at rest in $\mathrm{W}$ and thus moving in a uniform translational manner relative to $\mathrm{W}^{\prime}$ and that its length as measured in $\mathrm{W}^{\prime}$ is 5 units. Allow that in line with Einstein's criterion, a light flash begins in event A at one end of the rod, travels to event I where it reaches the other end of the rod, and is immediately reflected back. (Event I occurs on the world line of the end of the rod toward which the light flash travels after first being emitted.) The light flash returns to the end of the rod that it originated from in event $\mathrm{F}$ at $x=0$. In terms of the time of $\mathrm{W}^{\prime}, t_{\mathrm{AI}}^{\prime} \neq t_{\mathrm{IF}}^{\prime}$ and thus Einstein's original criterion for establishing simultaneity is not met in $\mathrm{W}$ when this process is considered in terms of the time of W'. From Figure 13, it can also be seen that $t_{\mathrm{AI}} \approx \mathrm{t}_{\mathrm{IF}} \approx 6$ units and that simultaneity, and thus time, is established in $\mathrm{W}$ in accordance with Einstein's original criterion for simultaneity in an inertial reference frame.

In terms of the time of $\mathrm{W}, t$, the passage of the light ray from one end of the rod to the other and back again occurs in about 12 units of time. If the concern is with the amount of time that elapses in W', the "stationary" reference frame, corresponding to these 12 units in $\mathrm{W}$, the "moving" reference frame, the dashed line parallel to the space axis for $\mathrm{W}^{\prime}$ indicates that the corresponding time in $\mathrm{W}^{\prime}$ is between 13 and 14 units.

Nothing physical is presented in the special theory to distinguish the different scenarios in the argument on the relativity of simultaneity. All that distinguishes them is an arbitrary choice on the part of the individual arguing the relativity of simultaneity concerning the direction in which the argument is made. This arbitrary choice is anchored in the experience of observers that they are at rest in their respective inertial reference frames in uniform translational motion relative to one another and that each of their respective inertial reference frames is for them "stationary" while the other reference frame is "moving." This arbitrary choice concerning the direction in which to argue the relativity of simultaneity is made implicitly when an individual is concerned with other results of the special theory, most importantly the temporal duration of 


\section{Chapter 3}

occurrences or the spatial length of physical existents in inertial reference frames in uniform translational motion relative to one another. Other results of the special theory depend on these spatial and temporal relations (Snyder, 1994). (Please see Appendix D for another approach to the link between cognition and the physical world in the special theory implied in the use of the invariant velocity of light to define simultaneity in an inertial reference frame.)

The Reference Frame

in the Special Theory

Consider the progression from Newtonian mechanics to the special theory, and the multitude of possible expressions of the spatial and temporal characteristics of some physical object or occurrence in the special theory that are dependent on the uniform translational velocity of the physical object or occurrence relative to an inertial reference frame. Whereas Newton considered space and time to exist on their own, it has been shown that in special relativity space and time depend on the particular inertial frame of reference in which they are considered. Earlier in the chapter it was noted that the length of a physical object, or the duration of a physical occurrence, depends on the uniform translational velocity that this object or occurrence has relative to the particular reference frame in which the length of the object, or the duration of the occurrence, is considered. In the special theory, rods moving in a uniform translational manner are shorter than they are at rest, and moving clocks moving in a uniform translational manner measure shorter durations for an occurrence than they would if they were at rest.

There are an unlimited number of uniform translational velocities that an object or occurrence can maintain relative to some inertial reference frame. Thus, there are an unlimited number of possible lengths of objects and durations of occurrences, with each such possible length or duration tied to some inertial reference frame moving at a particular uniform translational velocity relative to the object or occurrence. It has also been shown that space and time in an inertial reference frame depends on whether the reference frame is designated the "stationary" reference frame or the "moving" reference frame in the argument on the relativity of simultaneity.

Is it possible that the physical world is solely responsible for this multitude of possible expressions of length and duration in special relativity? This position is untenable because of the importance of the reference frame, and its associated temporal coordinate system, to spatial extension and temporal 


\section{The Special Theory}

duration. As has been stated, a reference frame and its associated temporal coordinate system are in part inventions of the human mind. The significance of the reference frame in the special theory is undeniable because spacetime in the special theory depends on the particular reference frame from which the physical world is considered. Indeed, the reference frame of an observer depends on his experience of being at rest in that reference frame.

The Significance of the

Observer's Being at Rest in an

Inertial Reference Frame in the Special Theory

It is also important to discuss the significance of observers considering themselves at rest in their respective inertial reference frames in uniform translational motion relative to one another in the special theory. An observer's considering himself at rest in his inertial reference frame is central to his considering his inertial reference frame at rest and thus able to act as the "stationary" inertial reference frame in the argument on the relativity of simultaneity. Without observers considering themselves at rest in their respective inertial reference frames, the relativity of simultaneity could not be argued with either reference frame considered the "stationary" inertial reference frame in the argument. There would be a preferred inertial reference frame for the description of physical phenomena if this principle did not hold. The preferred frame of reference would be that inertial reference frame in which an observer considered himself at rest, while in the other inertial reference frame the observer would consider himself moving at a uniform translational velocity relative to the preferred reference frame.

Observers, at rest in their respective inertial reference frames, have unique points of view even though physical phenomena can be described in the same way from either reference frame. Observers at rest in their inertial reference frame and who maintain that their reference frame is "stationary" will never be considered to be "stationary" by observers at rest in an inertial reference frame moving in a uniform translational manner relative to the former reference frame. The former reference frame, and its observer, will always be considered moving. Observers at rest in their own inertial reference frames will obtain an equivalent spatiotemporal structure regarding existents and occurrences in their own reference frames. But when they consider the other observers' reference frame, they will see the others' reference frame as "moving" and will find the spatiotemporal structure in that reference frame to be that characteristic for "moving" reference frames. The observer who considers 


\section{Chapter 3}

his reference frame "stationary" will always be the "moving" reference frame for observers at rest in the other inertial reference frame in uniform translational motion relative to it.

The possibilities of the spatiotemporal relationships between inertial reference frames are very great. Observers at rest in their inertial reference frames cannot rely on their physical measuring instruments alone to be the foundation of their measurements. There are simply too many possibilities for the specifications of the instruments that depend on the motion of an observer's inertial reference frame relative to the instruments. We have seen that the spatiotemporal structure of the reference frame itself has a significant cognitive component, both generally and in the special theory where spatiotemporal structure depends on which inertial reference frame is "stationary" and which "moving" in the argument on the relativity of simultaneity. Indeed, an observer's experience of being at rest in his reference frame is associated with the integrity of the special theory.

In the special theory, theoretical considerations are grounded in the experience of observers. The choice in which direction to argue the relativity of simultaneity when there are observers at rest in either of two inertial reference frames in uniform translational motion relative to one another is dependent on observers considering themselves at rest in their respective reference frames and their reference frames "stationary." The flexibility in an observer's reference frame is ultimately the basis for the choice as to which inertial reference frame is "stationary" or "moving" in arguing the relativity of simultaneity. 


\section{Chapter 4}

\section{The General Theory of Relativity}

After developing the special theory, Einstein questioned why inertial reference frames are granted special consideration vis-à-vis the laws of physics. Why aren't other reference frames, such as those that are accelerating, granted the same consideration? Einstein's thinking was similar to his consideration of the ether in his development of the special theory. The ether was supposed to be something physical, and yet physicists could find no evidence for its existence. The ether served physicists in their attempt to maintain the Newtonian view of the world. In the case of inertial reference frames, there was nothing that could be pointed to that supported the privileged position of these reference frames in regard to the laws of physics (Einstein, 1917/1961).

Essentially, Newton's laws are formulated for inertial reference frames, and there is basically nothing other than these laws that delineate inertial from non-inertial reference frames. That accelerated motions are the same in all inertial reference frames in Newtonian mechanics was demonstrated in Chapter 2 in the derivation of the invariance of Newton's second law of motion in inertial reference frames in uniform translational motion relative to one another. Thus, it was thought that accelerated motions are absolute and can distinguish inertial from non-inertial reference frames in these mechanics. Note, though, that this reliance on accelerated motions already depends on having delineated inertial reference frames in order to then distinguish them from non-inertial reference frames (Born, 1924/1965; Einstein \& Infeld, 1938/1966). ${ }^{6}$ There are other critical reasons why inertial reference frames are not preferred.

First, consider a small area of the earth an inertial reference frame in a uniform gravitational field. For an elevator freely falling to the earth and thus accelerating in a uniform manner, one can consider the inside of the freely falling elevator to be a local (i.e., over a small area) inertial reference frame in which the laws of physics hold. For an observer in the elevator, all of Newton's laws of motion would hold so long as objects did not come into contact with the walls of the elevator (Einstein \& Infeld, 1938/1966). Thus, the supposedly absolute character of the uniformly accelerated motion of the

\footnotetext{
${ }^{6}$ It should be remembered that Newton's first law of motion states that in the absence of the application of an external force, a physical body either is at rest or maintains a uniform, rectilinear motion. A frame of reference in which this law holds is an inertial frame of reference.
} 


\section{Chapter 4}

elevator does not affect the validity of Newton's laws for the man inside the elevator.

Further, Einstein (1916/1952, 1922/1956, 1917/1961) and Einstein and Infeld (1938/1966) pointed out that an inertial reference frame in a uniform gravitational field would be equivalent with regard to the motion of bodies to a uniformly accelerating frame of reference. Einstein's (1917/1961) example was that of a man in a large chest resembling a room and without windows deep in outer space that is accelerating uniformly due to its being towed by a rope connected to one end of the chest. According to Einstein, the man in the chest would consider himself in an inertial reference frame in a uniform gravitational field. The man in the chest would feel the same pull toward the floor that he would in such an inertial reference frame. Also, if he dropped an object, it would fall to the floor of the chest in the same manner as if the chest were in an inertial reference frame in a uniform gravitational field. It would accelerate in a uniform manner. Essentially, Einstein asked, "What is so special about inertial reference frames when one cannot even distinguish them in certain instances from other reference frames?" His answer was, "Nothing," and thus the laws of physics should hold for all frames of reference, not just inertial ones.

In response to these circumstances, Einstein set about formulating the laws of physics such that they were not limited to inertial reference frames (Einstein, 1949/1969). In order to accomplish this task, Einstein needed to determine the nature of spacetime in accelerating reference frames and inertial reference frames in gravitational fields. The results of special relativity were useful in this task.

Specifically, a uniformly accelerating reference frame can be considered a series of local inertial reference frames, each moving at a slightly different uniform translational velocity relative to the reference frame next to it. In this manner, Einstein could use the results he had obtained in special relativity in determining the nature of spacetime in the uniformly accelerating reference

frame. Recall that a unit measuring rod is shorter when it is moving at a uniform translational velocity in an inertial reference frame than when it is at rest in an inertial reference frame, and that the length of this rod is determined by its particular velocity. Thus, one would expect that for a very small unit measuring rod in a uniformly accelerating frame of reference, its length would vary depending on its particular velocity relative to the inertial reference frame from which the overall uniform acceleration of the accelerating reference frame is determined. If one considers many of these rods in close proximity to one 


\section{The General Theory}

another, the uniform translational velocities of the rods vary in a continuous manner and the rods measure progressively shorter, for example, as their velocities increase. A similar argument can be made for measuring the duration of some event in a uniformly accelerating reference frame. On the basis of this reasoning, Einstein argued that spacetime in accelerating reference frames is curved and not Euclidean in nature (e.g., [Einstein, 1917/1961]).

Due to the equivalence of a uniformly accelerating reference frame and an inertial reference frame in a uniform gravitational field, the results obtained for the former reference frame are applicable to the latter. Einstein applied these results to non-uniformly accelerating reference frames and inertial frames of reference in non-uniform gravitational fields, such as that associated with the earth.

\section{The Principle of Equivalence}

A closer look at the equivalence of inertial and non-inertial reference frames as regards the description of physical phenomena and its implications is warranted because the principle of equivalence illustrates the importance of mind in the general theory. It does so because of:

1) the nature of the principle of equivalence itself;

2) the reliance on the special theory locally for the development of spacetime curvature (which can be introduced because the principle of equivalence allows for gravitational fields to be described locally by the special theory);

3) the importance of an observer's being at rest in a reference frame.

In The Meaning of Relativity, Einstein stated the principle of equivalence in the following way and indicated how it provides the theoretical underpinning for the equality of inertial and gravitational mass:

Let now $\mathrm{K}$ be an inertial system. Masses which are sufficiently far from each other and from other bodies are then, with respect to $\mathrm{K}$, free from acceleration. We shall also refer these masses to a system of co-ordinates $\mathrm{K}^{\prime}$, uniformly accelerated with respect to $\mathrm{K}$. Relatively to $\mathrm{K}^{\prime}$ all the masses have equal and parallel accelerations; with respect to $\mathrm{K}^{\prime}$ they behave just as if a gravitational field were present and $\mathrm{K}^{\prime}$ were unaccelerated. 


\section{Chapter 4}

Overlooking for the present the question as to the "cause" of such a gravitational field...there is nothing to prevent our conceiving this gravitational field as real, that is, the conception that $\mathrm{K}^{\prime}$ is "at rest" and a gravitational field is present we may consider as equivalent to the conception that only $\mathrm{K}$ is an "allowable" system of co-ordinates and no gravitational field is present. The assumption of the complete physical equivalence of the systems of coordinates, $\mathrm{K}$ and $\mathrm{K}$ ', we call the "principle of equivalence;" this principle is evidently intimately connected with the law of the equality between the inert and the gravitational mass, and signifies an extension of the principle of relativity to co-ordinate systems which are in non-uniform motion relatively to each other. In fact, through this conception we arrive at the unity of the nature of inertia and gravitation. For according to our way of looking at it, the same masses may appear to be either under the action of inertia alone (with respect to $\mathrm{K}$ ) or under the combined action of inertia and gravitation (with respect to $\mathrm{K}^{\prime}$ ). (Einstein, 1922/1956, p. 57)

The principle of equivalence depends on observers considering themselves at rest in their respective reference frames. For the observer at rest in $K^{\prime}, K^{\prime}$ is at rest. We shall call the observer at rest in $\mathrm{K}^{\prime}$ "inside." $\mathrm{He}$ is at rest in his inertial reference frame that experiences a gravitational field and is observed by the observer "outside" at rest in K. For the observer at rest in K, $\mathrm{K}$ is at rest and the observer at rest in $\mathrm{K}^{\prime}$ is in an accelerating reference frame. The observer at rest in $\mathrm{K}$ observes the observer at rest in $\mathrm{K}^{\prime}$ from a different reference frame and associated temporal coordinate scheme than the observer at rest in K' relies on. The arbitrary character of the frame of reference, tied to an observer's being at rest in it, is what allows the observer "inside" in $\mathrm{K}^{\prime}$ and the observer "outside" in K to have equivalent descriptions of physical phenomena. Without this arbitrary character, dependent on the cognition of the observer, the possibility of different reference frames that yield equivalent descriptions would not be possible. The cognitive element in the particular reference frame employed by an observer, and the dependence of the particular reference frame on the experience of the observer being at rest that are significant in the special theory are no less important in the general theory. This point will gain more support when the nature of spacetime curvature in the general theory is discussed. 


\section{The General Theory}

Next, it should be shown how the principle of equivalence is the conceptual foundation for the equality of inertial and gravitational mass. One consequence is that a cognitive component in the principle of equivalence is tied directly to this equality.

\section{The Equality of Inertial and Gravitational Mass}

The principle of equivalence provides the theoretical foundation for the equality of inertial and gravitational mass, and this equality allows for the ingeneral equivalence between accelerating reference frames and inertial reference frames experiencing a gravitational field (Einstein, 1917/1961; Feynman, Leighton, \& Sands, 1964). Regarding a physical object, its acceleration in Newtonian mechanics is given by the equation

$$
f=m_{i} a
$$

where $f$ is the external force applied to a physical object, $m_{i}$ is the inertial mass of the object and is constant, and $a$ is the object's acceleration in response to the force. The inertial mass of an object does not depend on either the type of physical object or, in Newtonian mechanics, on the state of its motion. The gravitational force exerted on a physical object in Newtonian mechanics is given by the equation

$$
f=m_{g} i_{g},
$$

where $f$ is the force due to gravitation that is applied to a physical object, $m_{g}$ is its gravitational mass and is constant, and $i_{g}$ is the intensity of the gravitational field. As with inertial mass, the gravitational mass of a physical object does not depend on either the type of physical object or, in Newtonian mechanics, on the state of its motion. From these two equations the result can be derived

$$
a=\left(m_{g} / m_{i}\right)\left(i_{g}\right) \text {. }
$$

With the choice of a suitable constant, $a=i_{g}$. The acceleration of the physical object in a gravitational field is equal to the intensity of the field.

If the ratio of gravitational mass to inertial mass were not invariant and $a \neq i_{g}$, then the description of motion of physical bodies when described from a uniformly accelerating reference frame or an inertial reference frame experiencing a uniform gravitational field would not be equivalent. Further, the intensity of the gravitational field "inside" a reference frame that is uniformly accelerating relative to an inertial reference frame would not be directly proportional to the acceleration of objects "inside" the former reference frame. If the ratio of gravitational mass to inertial mass is invariant and $a=i_{g}$, the 


\section{Chapter 4}

magnitude of the uniform acceleration of the inertial reference frame experiencing a uniform gravitational field measured "outside" in the gravitationfree inertial reference frame is equal to the magnitude of the acceleration of objects "inside" the inertial frame of reference experiencing a gravitational field that is completely accounted for by the gravitational field. Without the invariant ratio of gravitational mass to inertial mass, the principle of equivalence would not hold as there would not be a uniform link between a gravitation-free inertial reference frame and an inertial reference frame experiencing a uniform gravitational field.

The principle of equivalence allows for the equivalence of an inertial reference frame with no gravitational field and an inertial reference frame with a gravitational field in the description of physical phenomena. The principle of equivalence is central in the development of spacetime curvature for gravitational fields.

\section{Deriving Spacetime Curvature}

In outlining the essentials of the general theory of relativity, Einstein discussed an example of how an observer in an accelerating reference frame would be affected in his measurements of temporal duration and how his measurements could be accounted for in terms of local Lorentz frames. He wrote:

Let us consider a space-time domain in which no gravitational field exists relative to a reference-body $\mathrm{K}$ whose state of motion has been suitably chosen. $\mathrm{K}$ is then a Galileian reference-body as regards the domain considered, and the results of the special theory of relativity hold relative to $\mathrm{K}$. Let us suppose the same domain referred to a second body of reference $\mathrm{K}^{\prime}$, which is rotating uniformly with respect to $\mathrm{K}$. In order to fix our ideas, we shall imagine $K^{\prime}$ to be in the form of a plane circular disc, which rotates uniformly in its own plane about its centre. An observer who is sitting eccentrically on the disc $\mathrm{K}^{\prime}$ is sensible of a force which acts outwards in a radial direction, and which would be interpreted as an effect of inertial (centrifugal force) by an observer who was at rest with respect to the original reference-body K. But the observer on the disc may regard his disc as a reference-body which is "at rest"; on the basis of the general principle of relativity he is justified in doing this [italics 


\section{The General Theory}

added]. This force acting on himself, and in fact on all other bodies which are at rest relative to the disc, he regards as the effect of a gravitational field. But since the observer believes in the general theory of relativity, this does not disturb him: he is quite in the right when he believes that a general law of gravitation can be formulated - a law which not only explains the motion of the stars correctly, but also the field of force experienced by himself.

The observer performs experiments on his circular disc with clocks and measuring-rods. In doing so, it is his intention to arrive at exact definitions for the signification of time- and spacedata with reference to the circular disc $K^{\prime}$, these definitions being based on his observations. What will be his experience in this enterprise?

To start with, he places one of two identically constructed clocks at the centre of the circular disc, and the other on the edge of the disc, so that they are at rest relative to it. We now ask ourselves whether both clocks go at the same rate from the standpoint of the non-rotating Galileian reference-body K. As judged from this body, the clock at the centre of the disc has no velocity, whereas the clock at the edge of the disc is in motion relative to $\mathrm{K}$ in consequence of the rotation....the latter clock goes at a rate permanently slower than that of the clock at the centre of the circular disc, i.e., as observed from $\mathrm{K}$. It is obvious that the same effect would be noted by an observer whom we will imagine sitting alongside his clock at the centre of the circular disc. Thus on our circular disc, or, to make the case more general, in every gravitational field, a clock will go more quickly or less quickly, according to the position in which the clock is situated (at rest). (Einstein, 1917/1961, pp. 79-81)

What is the mathematical relation between the rates of clocks at rest in either of two inertial reference frames moving in a uniform translational manner relative to one another? Consider the inertial reference frames $\mathrm{W}$ and $\mathrm{W}^{\prime}$ discussed in Chapter 3. If $\mathrm{W}^{\prime}$ is considered the "moving" inertial reference frame and $\mathrm{W}$ the "stationary" inertial reference frame, the relation between the rates of the clocks in $\mathrm{W}$ and $\mathrm{W}^{\prime}$ is given by

$$
\Delta t=\Delta t^{\prime} /\left(1-v^{2} / c^{2}\right)^{1 / 2} \text {. }
$$




\section{Chapter 4}

where $\Delta t$ is the duration of an occurrence in $\mathrm{W}, \Delta t^{\prime}$ is the duration of this same occurrence in $\mathrm{W}^{\prime}, v$ is the uniform translational velocity of $\mathrm{W}^{\prime}$ relative to $\mathrm{W}$, and $c$ is the invariant velocity light in any inertial reference frame. The relation between temporal occurrences is depicted in Figure 14 where $\Delta t^{\prime}=10$ units and $\Delta t=10 /\left(1-v^{2} / c^{2}\right)^{1 / 2}$ units. example?

And what of the observer's use of measuring rods in Einstein's

If the observer applies his standard measuring-rod...tangentially to the edge of the disc, then as judged from the Galileian system [i.e., K], the length of this rod will be less than 1 , since...moving bodies suffer a shortening in the direction of the motion.

(p. 81)

The mathematical relation between the spatial lengths of a rod in two inertial reference frames $\mathrm{W}$ and $\mathrm{W}^{\prime}$ and which is lying along the direction of uniform translational motion of $\mathrm{W}$ and $\mathrm{W}^{\prime}$ relative to one another is

$$
\Delta x=\Delta x^{\prime}\left(1-v^{2} / c^{2}\right)^{1 / 2}
$$

where $\Delta \mathrm{x}$ is the spatial length of a rod in the "stationary" reference frame W, $\Delta x^{\prime}$ is the spatial length of this same rod in the "moving" reference frame $\mathrm{W}^{\prime}$ where it is at rest, $v$ is the uniform translational velocity of $\mathrm{W}^{\prime}$ relative to $\mathrm{W}$, and $c$ is the invariant velocity light in any inertial reference frame. The relation between spatial lengths of a rod in $\mathrm{W}$ and $\mathrm{W}^{\prime}$ is depicted in Figure 15 where $\Delta x^{\prime}=10$ units and $\Delta x=10\left(1-v^{2} / c^{2}\right)^{1 / 2}$ units.

Spacetime curvature may be found for an accelerating frame, or an associated inertial reference frame in a gravitational field, essentially by deriving the pattern of special relativistic results that hold for the local Lorentz frames and that compose the global reference frame (Born, 1924/1965; Einstein, 1916/1952; Misner, Thorne, \& Wheeler, 1973).

\section{Further Comments on Extending}

the Results to Gravitational Fields

Misner, Thorne, and Wheeler (1973) summed up the relationship of the general and special theories of relativity in writing, "General relativity is built on special relativity" (p. 164). In elaborating on this statement, the authors wrote: 


\section{The General Theory}

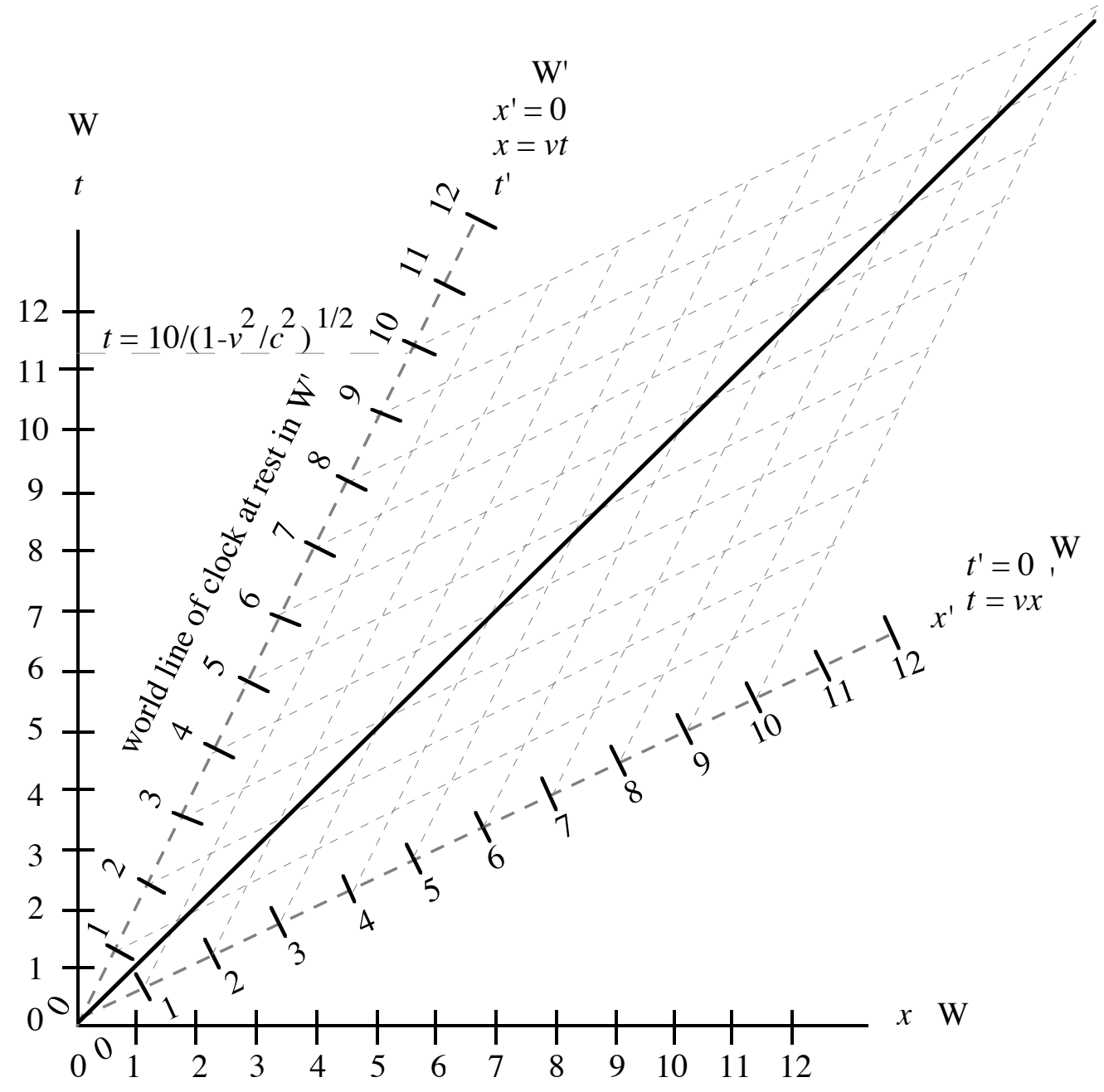

Figure 14

Minkowski diagram of $\mathrm{W}$ and $\mathrm{W}^{\prime}$ showing relation between time of "moving" clock at rest in $\mathrm{W}^{\prime}$ and time of clocks at rest in "stationary" reference frame W.

A tourist in a powered interplanetary rocket feels "gravity." Can a physicist by local effects convince him that this "gravity" is bogus? Never, says Einstein's principle of the local equivalence of gravity and accelerations. But then the physicist will make no errors if he deludes himself into treating true gravity as a local illusion caused by acceleration. Under this delusion, he barges 


\section{Chapter 4}

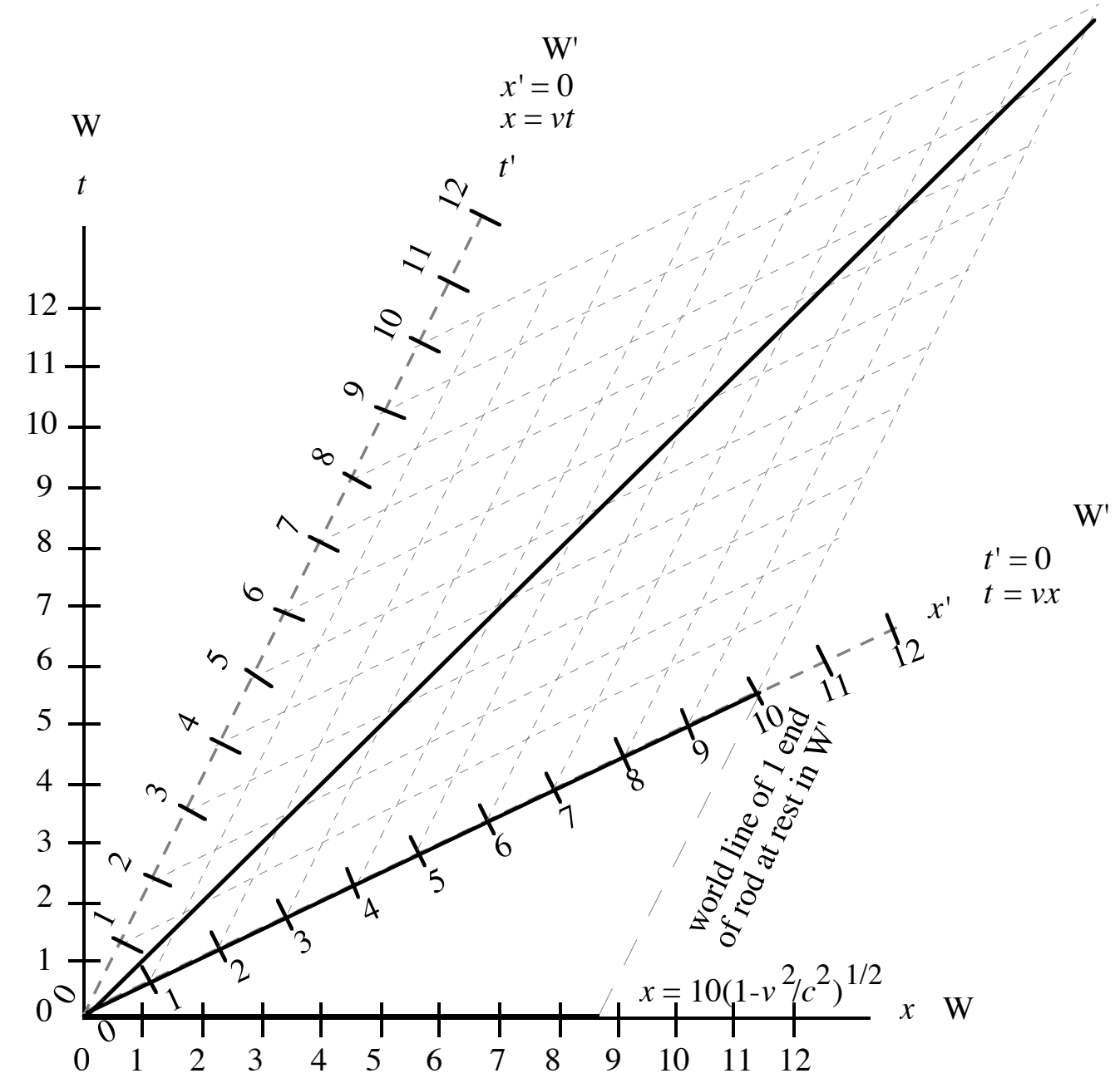

Figure 15

Minkowski diagram of $\mathrm{W}$ and $\mathrm{W}^{\prime}$ showing relation between spatial length of rod at rest in $\mathrm{W}^{\prime}$ and spatial length of "moving" rod in "stationary" reference frame W.

ahead and solves gravitational problems by using special relativity: if he is clever enough to divide every problem into a network of local questions, each solvable under such a delusion, then he can work out all influences of any gravitational field. Only three basic principles are invoked: special relativity physics, the equivalence principle, and the local nature of physics. They are simple and clear. To apply them, however, 


\section{The General Theory}

imposes a double task: (1) take spacetime apart into locally flat pieces (where the principles [of the special theory] are valid), and (2) put these pieces together again into a comprehensible picture. To undertake this dissection and reconstitution, to see curved dynamic spacetime inescapably take form, and to see the consequences for physics: that is general relativity. (p. 164)

We should see that concerns pertinent to the special theory remain relevant in the general theory as the general theory is clearly dependent on the special theory, in particular on relationships between local reference frames, Euclidean-like in nature, moving at uniform translational velocities relative to one another. When these local Euclidean-like reference frames are considered in their relation to one another, the same issues concerning the relativity of simultaneity and the nature of the reference frame in the special theory can be seen to be important in the general theory as well.

Misner, Thorne, and Wheeler attributed great significance to the principle of equivalence in the general theory, and their statement that "the physicist will make no errors if he deludes himself into treating true gravity as a local illusion caused by acceleration [italics added]" (p. 164) should not be lost in terms of its significance in implying that there is a cognitive element in the principle of equivalence.

\section{The Nature of the Gravitational Field}

From the foregoing considerations, it can reasonably be argued that the gravitational field exists only in so far as it is considered to exist by an appropriately placed observer. ${ }^{7}$ Einstein (1917/1961) objected to the scope of this last thesis. He wrote:

Now we might easily suppose that the existence of a gravitational field is always only an apparent one. We might also think that, regardless of the kind of gravitational field which may be present, we could always choose another reference-body such that no gravitational field exists with reference to it. This is by no means true for all gravitational fields, but only for those of quite special form. It is, for instance, impossible to choose a

\footnotetext{
${ }^{7}$ Indeed, one can legitimately raise the question of the nature of mass since a central feature of mass is its association with a gravitational field.
} 


\section{Chapter 4}

body of reference such that, as judged from it, the gravitational field of the earth (in its entirety) vanishes. (p. 69)

Though Einstein was correct in asserting that it is not possible to choose a single accelerating frame of reference such that the gravitational field of the earth vanishes, the gravitational field of the earth is equivalent to some pattern of local, uniformly accelerating frames of reference. In fact, the determination of spacetime curvature associated with the earth's gravitational field depends on this corresponding pattern of local, uniformly accelerating reference frames. Thus, Einstein's argument is correct but misleading. Einstein relied on the equivalence of inertial reference frames in gravitational fields of constant intensity and uniformly accelerating frames of reference for his formulations concerning all gravitational fields allowed in, and described by, general relativity. These formulations, of course, include the gravitational field associated with the earth. The intermediary between the gravitation-free inertial reference frames characteristic of special relativity and the inertial reference frames in gravitational fields characteristic of general relativity (these gravitational fields being the ultimate concern of general relativity) are accelerating frames of reference.

Moreover, the question must be raised, what in principle distinguishes an individual observer in different accelerating reference frames, such as the earth's gravitational field considered as a sequence of such reference frames, from an individual who is in only one uniformly accelerating reference frame? What is so unusual that distinguishes the former observer from the latter? Consider Einstein's own description of the experience of an observer who is at first in an inertial reference frame and then in an accelerating reference frame, one that in principle need not be uniformly accelerating. The example concerns a train traveling with uniform translational velocity along an embankment and then experiencing a non-uniform motion, the application of the brakes. Einstein wrote:

It is certainly true that the observer in the railway carriage experiences a jerk forwards as a result of the application of the brake, and that he recognises in this non-uniformity of motion (retardation) of the carriage. But he is compelled by nobody to refer this jerk to a "real" acceleration (retardation) of the carriage. He might also interpret his experience thus: "My body of reference (the carriage) remains permanently at rest. With reference to it, however, there exists (during the period of 


\section{The General Theory}

application of the brakes) a gravitational field which is directed forwards and which is variable with respect to time. Under the influence of this field, the embankment together with the earth moves non-uniformly in such a manner that their original velocity in the backwards direction is continuously reduced." [italics added] (Einstein, 1917/1961, pp. 69-70)

The equivalence of the options for the description of physical phenomena is the basis for the principle of equivalence. In the scenario proposed by Einstein, the train is an inertial reference frame that, for an observer at rest on the train, at some point in time experiences a gravitational field. For the observer on the train, this field is associated with the "nonuniform motion" of the embankment and earth. Is the experience of the observer in the railway carriage who considers that "the embankment together with the earth moves non-uniformly in such a manner that their original velocity in the backwards direction is continuously reduced [italics added]" significantly less difficult to accept than that a sequence of local uniformly accelerating reference frames, each accelerating in a different fashion, could not be interpreted as being a gravitational field?

With regard to uniformly accelerating reference frames which are equivalent to inertial reference frames in gravitational fields of uniform intensity, the arbitrary nature of the gravitational field is not in question. This is a straightforward expression of the general principle of relativity that is at the heart of the general theory, namely that frames of reference, whether or not they are inertial reference frames, are basically equivalent for formulating the laws of physics. With regard to the earth's gravitational field, Einstein was inconsistent because he: (1) relied on the special principle of relativity to understand the spacetime curvature associated with this gravitational field, and (2) did not fully acknowledge this reliance when he implied that the reference frame associated with the earth's gravitational field is essentially a preferred frame of reference. With regard to point 2, it is an arbitrary choice for an individual considering the spacetime curvature associated with the earth's gravitational field whether the physical phenomenon that can be considered a gravitational field is considered as such or whether it is considered a particular pattern of accelerating frames of reference.

The observer "inside" the inertial reference frame experiencing a gravitational field like that of the earth considers himself at rest in accordance with the general theory and with the special theory upon which the 


\section{Chapter 4}

spatiotemporal relations of this observer's overall reference frame are developed. The observer "outside" in an inertial reference frame free of any gravitational field and who considers the observer "inside," considers the observer to be located in a pattern of accelerating reference frames. The "outside" observer does not have a problem accomplishing this. The inertial reference frame with the gravitational field for the observer "inside" can be completely transformed by the observer "outside." The physicist, or other individual, considering spacetime curvature can maintain that it is explained either by the observer who is "inside" or the observer who is "outside." There are different vantage points for the observer "inside" and "outside." The ability to maintain these different vantage points is tied to the physicist's ability to see these vantage points as equivalent with regard to the description of physical phenomena. The quote from Misner, Thorne, and Wheeler presented above is relevant here, as is a quote from Resnick (1968).

A more general theory [of relativity compared to the special theory of relativity] is needed which takes into account the principle of equivalence and which generalizes even that principle to nonuniform (inhomogeneous) gravitational fields....First, inhomogeneous gravitational fields can be transformed away, or imitated, by having at each point in the field a different accelerated frame that replaces the local (infinitesimal) field there. In such local frames, the special theory of relativity is valid so that the invariance of the laws of physics under a Lorentz transformation applies to infinitesimal regions. Second, through an invariant space-time metric that follows from this, we can link geometry to gravitation and geometry becomes non-Euclidean. That is, the presence of a large body of matter causes space-time to warp in the region near it so that space-time becomes non-Euclidean. This warping is equivalent to the gravitational field. The curvature of space-time in general relativity replaces the gravitational field of classical theory. Hence, the geometry of space-time is determined by the presence of matter. In this sense, geometry becomes a branch of physics. The fact that special relativity is valid in small regions corresponds to the fact that Euclidean geometry is valid over small parts of a curved surface. In large regions, special relativity and Euclidean geometry need not apply so that the world lines of light rays and inertial motion need not be straight; 


\section{The General Theory}

instead, they are geodetic, that is, as straight as possible. (pp.

215-216)

Resnick held to the position that the geometry of spacetime is determined by the presence of matter. But, in his quote, it is clear that an equivalent statement is that the geometry of spacetime is determined by some pattern of accelerating frames of reference. Notice Resnick's use of the words transformed, imitated, replaces, and equivalent, and the sentences in which they are used. The existence of the gravitational field is an arbitrary choice by the physicist considering the vantage points of observers "inside" and "outside" the inertial reference frame experiencing a gravitational field. The general equivalence of accelerating frames of reference and inertial reference frames in gravitational fields indicates that the existence of gravitational fields depends on the different perspectives on these "inside" and "outside" observers.

The Significance of the Observer's Being at Rest in a Reference Frame in the General Theory

To review, in general relativity, a uniformly accelerating reference frame is equivalent with regard to the description of motion of a physical body to an inertial reference frame in a gravitational field of uniform intensity. It is this equivalence that provides the theoretical foundation for the equivalence of inertial mass in Newton's laws of motion and gravitational mass in Newton's law of gravitation. The principle of equivalence underlies the general principle of relativity that states that the laws of physics apply to reference frames whether or not they are inertial ones. In outlining the essentials of the general theory of relativity, Einstein's discussion of a situation like that of the amusement park ride found in Chapter 2 was presented in which the importance of the observer's being at rest in his reference frame in the description of physical phenomena was noted explicitly by Einstein. Specifically, Einstein's discussion concerned the reference bodies $\mathrm{K}$ and $\mathrm{K}^{\prime}$ discussed earlier, where $\mathrm{K}$ acts as the anchor for an inertial reference frame and $\mathrm{K}^{\prime}$ is a plane circular disk rotating in a uniform manner relative to $\mathrm{K}$. In quoting from Einstein's discussion, the observer at the center of $\mathrm{K}^{\prime}$ :

may regard his disc as a reference-body which is "at rest"; on the basis of the general principle of relativity he is justified in doing this. This force acting on himself, and in fact on all other bodies which are at rest relative to the disc, he regards as the effect of a gravitational field. (Einstein, 1917/1961, p. 80) 


\section{Chapter 4}

It has been emphasized in the preceding chapters that an essential characteristic of the observer's reference frame is that he is at rest in it. Thus, in the above example, rather than considering himself accelerating, the observer on the disc considers himself stationary in a gravitational field. If the disc moved differently than did the observer, the observer would use a different reference body that would be associated with his reference frame, one that was moving in the same manner as himself. When Einstein focused on the rotating disc being a reference body at rest for the observer sitting eccentrically on the disc, he essentially was concerned with this observer's being at rest in his reference frame for which the disc was the associated reference body.

As just indicated, the observer's being at rest in a reference frame is no less a significant factor in the general theory than it is in the special theory. Further, it has been shown that the origination of spacetime curvature in the general theory depends on the application of special relativistic results locally, that is to accelerating reference frames considered as a sequence of local inertial reference frames moving at various uniform translational velocities. Thus, the importance of being at rest in inertial reference frames in the special theory remains important in the local reference frames that comprise accelerating reference frames.

An examination of the conceptual foundation of the general theory has indicated that cognition is linked directly to the physical world and indeed has an impact with regard to its spatiotemporal structure and functioning. We have seen the significance of cognition in: 1) the nature of the principle of equivalence itself; 2) the reliance on the special theory locally for the development of spacetime curvature; and 3) the importance of an observer being at rest in a reference frame.

It should be pointed out that the significance of cognition to the physical world in the general theory ultimately depends on the flexibility in an observer's reference frame and associated temporal coordinate scheme. The importance of this flexibility for the special theory has been emphasized. In the general theory, this flexibility allows for the results of the special theory to hold locally and for various types of reference frames to be regarded as equivalent concerning the description of physical phenomena. This flexibility is not dependent on the physical phenomena that are described within a reference frame and its associated temporal coordinate structure. In contrast to the lack of flexibility in spatiotemporal structure implicit in absolute space and time, in the 


\section{The General Theory}

special and general theories, this flexibility allows for cognition to be linked to spatiotemporal structure. 


\section{Chapter 5}

\section{Quantum Mechanics}

Quantum mechanics is fundamentally a theory concerned with knowledge of the physical world. It is not fundamentally concerned with describing the functioning of the physical world independent of the observing, thinking person, as Newtonian mechanics is generally considered to be (Snyder, 1990, 1992c). Chief among the reasons for the thesis that cognition and the physical world are linked in quantum mechanics is that all knowledge concerning physical existents is developed using their associated wave functions, and the wave functions provide only probabilistic knowledge regarding the physical world (Liboff, 1993). There is no physical world in quantum mechanics that is assumed to function independently of the observer who uses quantum mechanics to develop predictions and who makes observations that have consistently been found to support these predictions. Also significant is the immediate change in the quantum mechanical wave function associated with a physical existent that generally occurs throughout space upon measurement of the physical existent. This change in the wave function is not limited by the velocity limitation of the special theory of relativity for physical existents-the velocity of light in vacuum.

Another relevant feature of quantum mechanics is the complex number nature of the wave function associated with a physical existent that is the basis for deriving whatever information can be known concerning the existent (Eisberg \& Resnick, 1974/1985). A complex function is one that has both mathematically imaginary and real components. The physical world is traditionally described by mathematically real numbers, giving rise to Eisberg and Resnick's (1974/1985) comment that "we should not attempt to give to wave functions [in quantum mechanics] a physical existence in the same sense that water waves have a physical existence" (p. 147).

Nonetheless, the particular demonstration concerning the phenomenon of interference to be discussed in the next section is remarkable. Examining interference will spotlight the wave-particle duality in quantum mechanics, the key feature of this duality being that physical existents sometimes show particlelike characteristics and sometimes show wave-like characteristics. Wave functions exhibiting interference are based on the sum of two or more elementary wave functions. In contrast, where interference does not 


\section{Quantum Mechanics}

characterize some physical phenomenon, this phenomenon is described by a wave function that consists of only one of these elementary wave functions.

Feynman's Two-Hole Gedankenexperiments

Generally the change in the wave function that often occurs in measurement in quantum mechanics has been ascribed to the unavoidable physical interaction between the measuring instrument and the physical entity measured. Indeed, Bohr (1935) maintained that this unavoidable interaction was responsible for the uncertainty principle, more specifically the inability to simultaneously measure observable quantities described by non-commuting Hermitian operators (e.g., the position and momentum of a particle). The following series of gedankenexperiments in this section will show that this interaction is not necessary to effect a change in the wave function. The series of gedankenexperiments indicates that knowledge plays a significant role in the change in the wave function that often occurs in measurement (Snyder, 1996a, 1996b).

\section{Gedankenexperiment 1}

Feynman, Leighton, and Sands (1965) explained that the distribution of electrons passing through a wall with two suitably arranged holes to a backstop where the positions of the electrons are detected exhibits interference (Figure 16). Electrons at the backstop may be detected with a Geiger counter or an electron multiplier. Feynman et al. explained that this interference is characteristic of wave phenomena and that the distribution of electrons at the backstop indicates that each of the electrons acts like a wave as it passes through the wall with two holes. It should be noted that when the electrons are detected in this gedankenexperiment, they are detected as discrete entities, a characteristic of particles, or in Feynman et al.'s terminology, "lumps" (p. 1-5).

In Figure 16, the absence of lines indicating possible paths for the electrons to take from the electron source to the backstop is not an oversight. An electron is not taking one or the other of the paths. Instead, the wave function associated with each electron after it passes through the holes is the sum of two more elementary wave functions, with each of these wave functions experiencing diffraction at one or the other of the holes. Epstein (1945) emphasized that when the quantum mechanical wave of some physical entity such as an electron exhibits interference, it is interference generated only in the wave function characterizing the individual entity. 


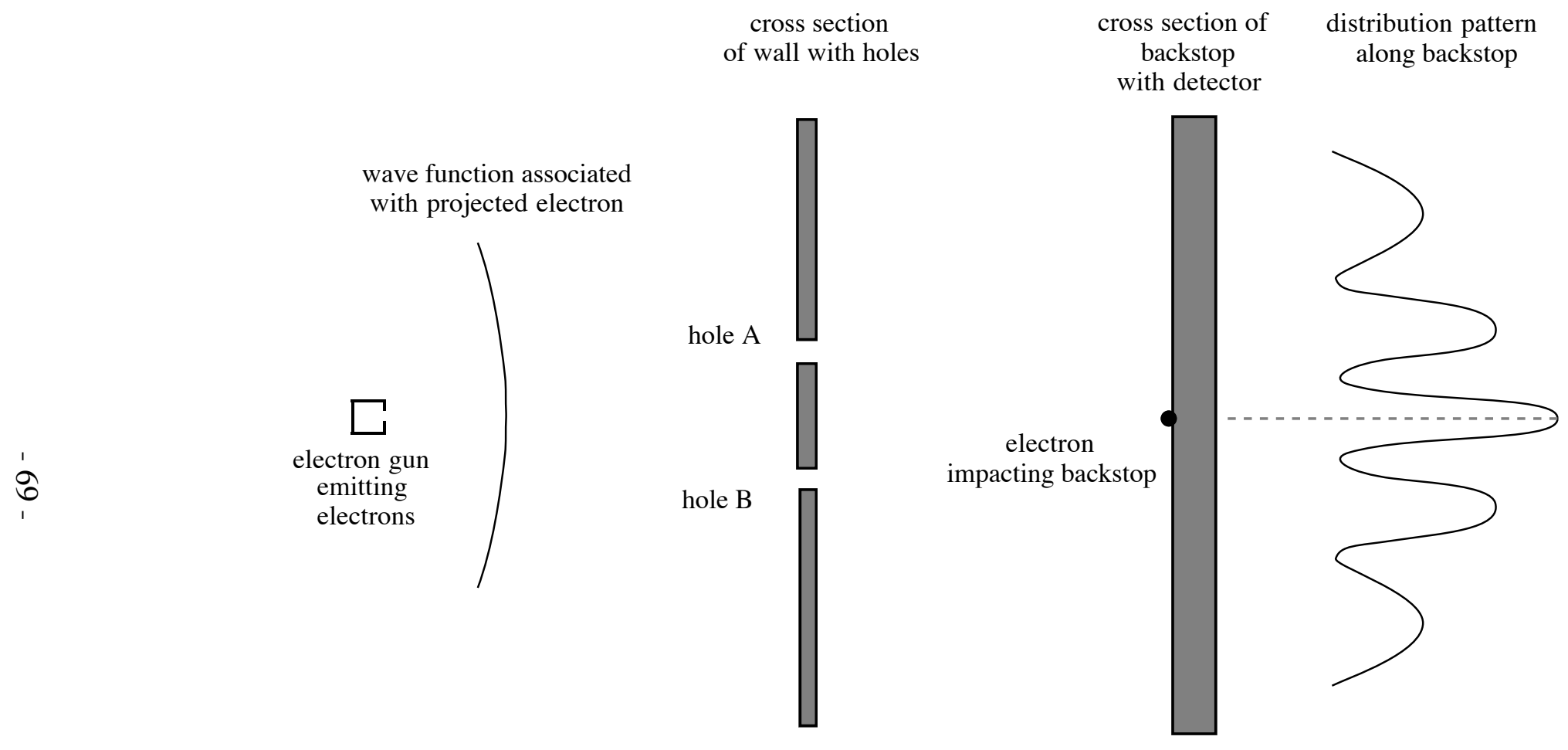

Figure 16

Two-hole gedankenexperiment in which the distribution of electrons reflects interference in the wave functions of electrons.

(Gedankenexperiment 1) 


\section{Quantum Mechanics}

The diffraction patterns resulting from the waves of the electrons passing through the two holes would at different spatial points along a backstop behind the hole exhibit constructive or destructive interference. At some points along the backstop, the waves from each hole sum (i.e., constructively interfere), and at other points along the backstop, the waves from each hole subtract (i.e., destructively interfere). The distribution of electrons at the backstop is given by the absolute square of the combined waves at different locations along the backstop, similar to the characteristic of a classical wave whose intensity at a particular location is proportional to the square of its amplitude. Because the electrons are detected as discrete entities, like particles, at the backstop, it takes many electrons to determine the intensity of the quantum wave that describes each of the electrons and that is reflected in the distribution of the electrons against the backstop.

\section{Gedankenexperiment 2}

Feynman et al. further explained that if one were to implement a procedure in which it could be determined through which hole the electron passed, the interference pattern is destroyed and the resulting distribution of the electrons resembles that of classical particles passing through the two holes in an important way. Feynman et al. relied on a strong light source behind the wall and between the two holes that illuminates an electron as it travels through either hole (Figure 17). Note the significant difference between the distribution patterns in Figures 16 and 17.

In Figure 17, the path from the electron's detection by the light to the backstop is indicated, but it is important to emphasize that this path is inferred only after the electron has reached the backstop. A measurement of the position of the electron with the use of the light source introduces an uncertainty in its momentum. Only when the electron is detected at the backstop can one infer the path the electron traveled from the hole it went through to the backstop. It is not something one can know before the electron strikes the backstop.

In Feynman et al.'s gedankenexperiment using the light source, the distribution of electrons passing through both holes would be similar to that found if classical particles were sent through an analogous experimental arrangement in an important way. Specifically, as in the case of classical particles, this distribution of electrons at the backstop is the simple summation of the distribution patterns for electrons passing through one or the other of the holes. Figure 18 shows the distribution patterns of electrons passing through 


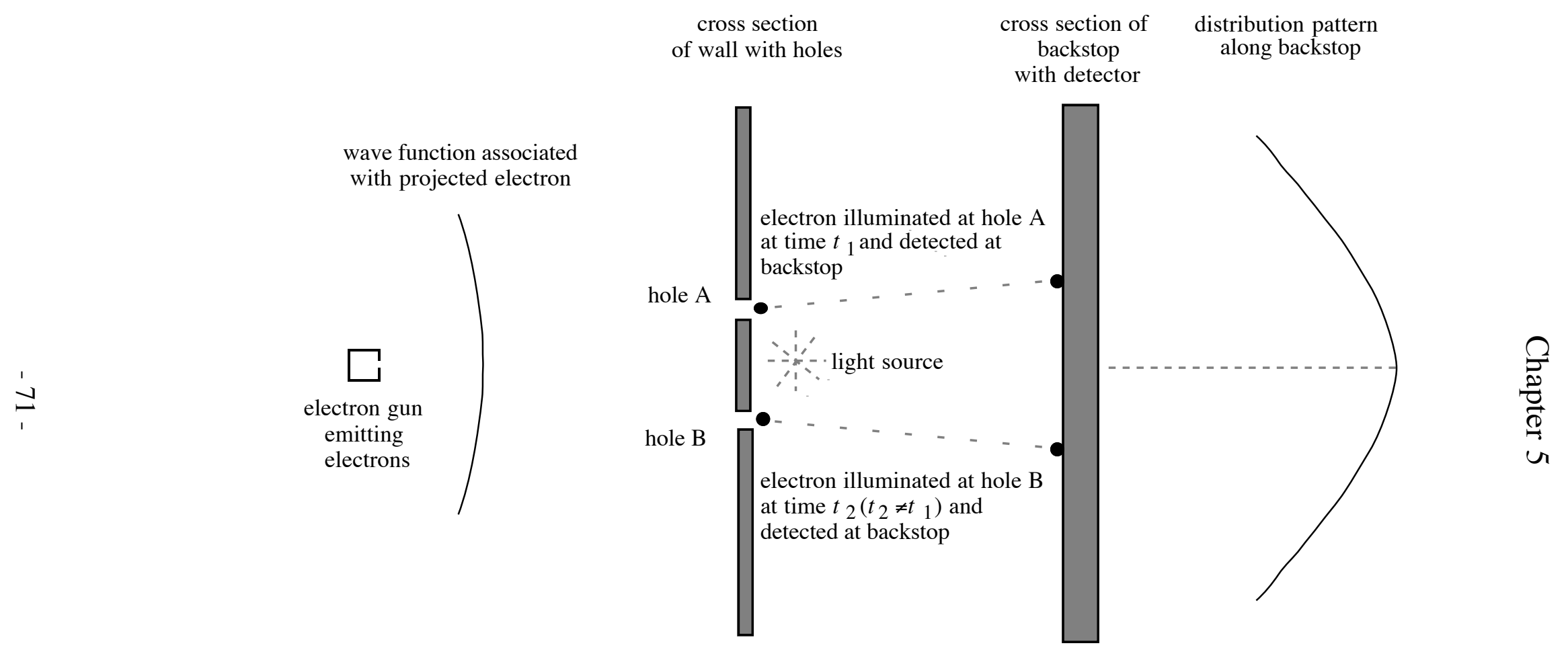

Figure 17

Two-hole gedankenexperiment with strong light source. (Gedankenexperiment 2) 
cross section
of wall with holes

wave function associated with projected electron

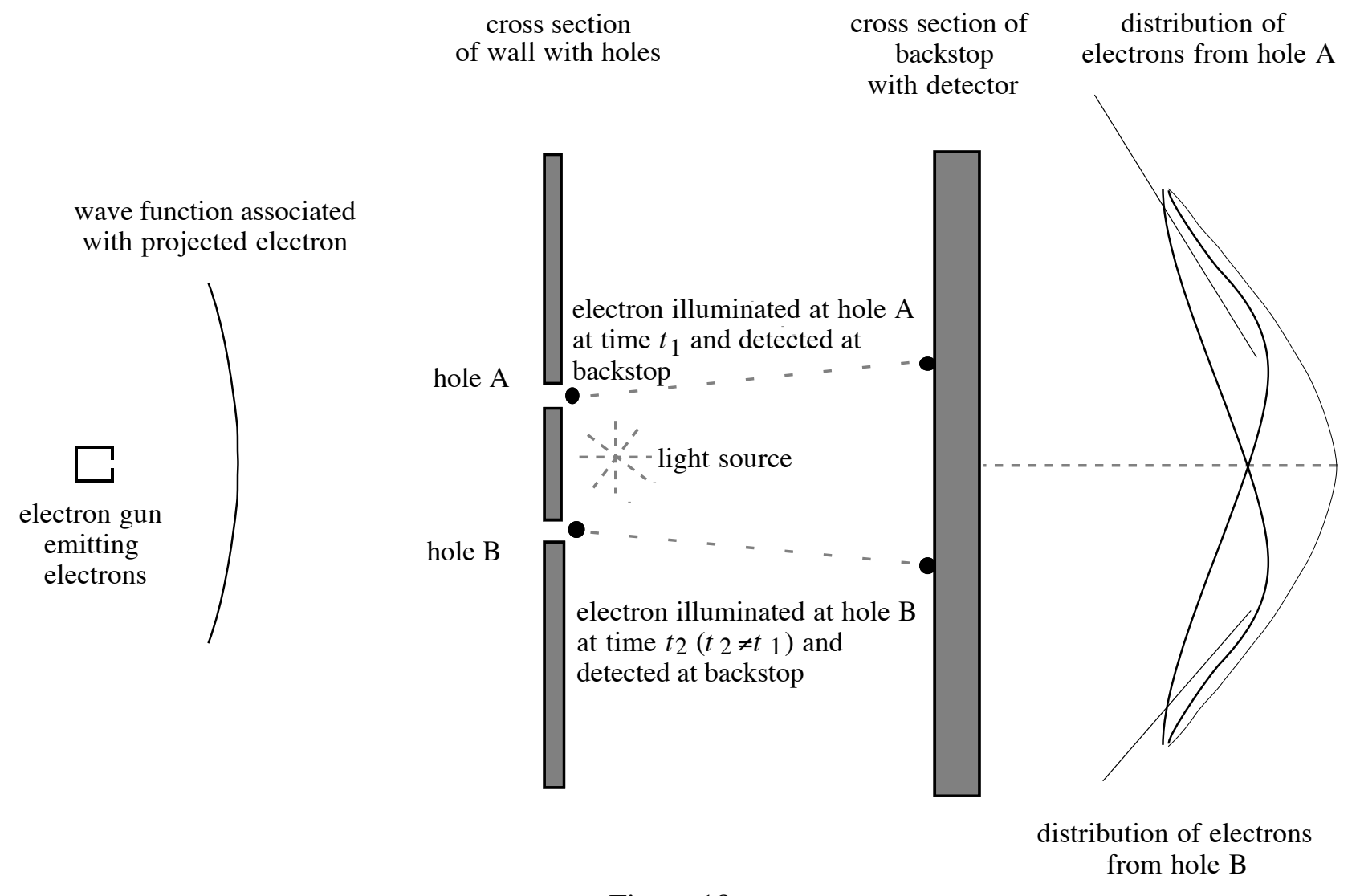

Figure 18

Two-hole gedankenexperiment with strong light source in which the distribution of electrons from each hole is shown. 


\section{Chapter 5}

hole A and electrons passing through hole B in Gedankenexperiment 2. These distribution patterns are identical to those that would occur if only one or the other of the holes were open at a particular time. An inspection of Figure 18 shows that summing the distribution patterns for the electrons passing through hole $\mathrm{A}$ and those passing through hole $\mathrm{B}$ results in the overall distribution of electrons found in Gedankenexperiment 2.

\section{The Uncertainty Principle}

Feynman et al.'s gedankenexperiments are themselves very interesting in that they illustrate certain apparently incongruent characteristics of microscopic physical existents, namely particle-like and wave-like features. Feynman et al. discussed their gedankenexperiments in terms of Heisenberg's uncertainty principle. Feynman et al. wrote:

He [Heisenberg] proposed as a general principle, his uncertainty principle, which we can state in terms of our experiment as follows: "It is impossible to design an apparatus to determine which hole the electron passes through, that will not at the same time disturb the electrons enough to destroy the interference pattern." If an apparatus is capable of determining which hole the electron goes through, it cannot be so delicate that it does not disturb the pattern in an essential way. (p. 1-9)

Note that Feynman et al. implied in their description of the uncertainty principle that there is an unavoidable interaction between the measuring instrument (in their gedankenexperiment, the strong light source emitting photons) and the physical entity measured. Feynman et al. also wrote concerning Gedankenexperiment 2:

the jolt given to the electron when the photon is scattered by it is such as to change the electron's motion enough so that if it might have gone to where $P_{12}$ [the electron distribution] was at a maximum [in Gedankenexperiment 1] it will instead land where $P_{12}$ was at a minimum; that is why we no longer see the wavy interference effects. (p. 1-8)

In determining through which hole an electron passes, Feynman et al., like most physicists, maintained that the electrons are unavoidably disturbed by the photons from the light source and it is this disturbance by the photons that destroys the interference pattern. Indeed, in a survey of a number of the textbooks of quantum mechanics, it is interesting that each author, in line with 


\section{Quantum Mechanics}

Feynman and Bohr, allowed a central role in the change in the wave function that occurs in a measurement to a physical interaction between the physical existent measured and some physical measuring apparatus. The authors of these textbooks are Dicke and Witke (1960), Eisberg and Resnick (1974/1985), Gasiorowicz (1974), Goswami (1992), Liboff (1993), Merzbacher (1961/1970), and Messiah (1962/1965).

It is important to note explicitly that some causative factor is necessary to account for the very different distributions of the electrons in Figures 16 and 17. Feynman et al. maintained that the physical interaction between the electrons and photons from the light source is this factor.

\section{Gedankenexperiment 3}

Feynman et al.'s gedankenexperiments indicate that in quantum mechanics the act of taking a measurement in principle is linked to, and often affects, the physical world which is being measured. The nature of taking a measurement in quantum mechanics can be explored further by considering a certain variation of Feynman et al.'s second gedankenexperiment (Epstein, 1945; Renninger, 1960). ${ }^{8}$ The results of this exploration are even more surprising than those presented by Feynman et al. in their gedankenexperiments. Empirical work on electron shelving that supports the next gedankenexperiment has been conducted by Nagourney, Sandberg, and Dehmelt (1986), Bergquist, Hulet, Itano, and Wineland (1986), and by Sauter, Neuhauser, Blatt, and Toschek (1986). This work has been summarized by Cook (1990). ${ }^{9}$

\footnotetext{
${ }^{8}$ Epstein (1945) presented the essence of Gedankenexperiment 3 using the passage of photons through an interferometer. Renninger (1960) also discussed a gedankenexperiment in an article entitled "Observations without Disturbing the Object" in which the essence of Gedankenexperiment 3 is presented.

${ }^{9}$ In electron shelving, an ion is placed into a superposition of two quantum states. In each of these states, an electron of the ion is in one or the other of two energy levels. The transition to one of the quantum states occurs very quickly and the transition to the other state occurs very slowly. If the ion is repeatedly placed in the superposition of states after it transitions to one or the other of the superposed states, one finds the atomic electron in general transitions very frequently between the superposed quantum states and the quantum state characterized by the very quick transition. The photons emitted in these frequently occurring transitions to the quantum state characterized by the very quick transition are associated with resonance fluorescence of the ion. The absence of resonance fluorescence means that the ion has transitioned into the quantum state that occurs infrequently.

Cook (1990) has pointed out that in the work of Dehmelt and his colleagues on electron shelving involving the $\mathrm{Ba}^{+}$ion, the resonance fluorescence of a single ion is of sufficient
} 


\section{Chapter 5}

In a similar arrangement to that found in Gedankenexperiment 2, one can determine which of the two holes an electron went through on its way to the backstop by using a light that is placed near only one of the holes and which illuminates only the hole it is placed by (Figure 19). Illuminating only one of the holes yields a distribution of the electrons similar to that which one would expect if the light were placed between the holes, as in Feynman et al.'s second gedankenexperiment. The distribution is similar to the sum of the distributions of electrons that one would expect if only one or the other of the holes were open at a particular time.

Moreover, when an observer knows that electrons have passed through the unilluminated hole because they were not seen to pass through the illuminated hole, the distribution of these electrons through the unilluminated hole resembles the distribution of electrons passing through the illuminated hole (Figure 20). Consider also the point that if: 1) the light is turned off before sufficient time has passed allowing the observer to conclude that an electron could not have passed through the illuminated hole, and 2) an electron has not been observed at the illuminated hole, the distribution of many such electrons passing through the wall is determined by an interference pattern that is the sum of diffraction patterns of the waves of the electrons passing through the two holes similar to that found in Gedankenexperiment 1 (Epstein, 1945; Renninger, 1960).

\section{Discussion of the Gedankenexperiments}

The immediate question is how are the results in Gedankenexperiment 3 possible given Feynman et al.'s thesis that physical interaction between the light source and electron is necessary to destroy the interference? Where the light illuminates only hole A, electrons passing through hole B do not interact with photons from the light source and yet interference is destroyed in the same manner as if the light source illuminated both holes A and B. In addition, the distribution of electrons passing through hole $\mathrm{B}$ at the backstop indicates that there has been a change in the description of these electrons, even though no physical interaction has occurred between these electrons and photons from the light source.

intensity to be detectable by the dark-adapted eye alone, and the making of a negative observation, to be discussed shortly, is thus not dependent on any measuring device external to the observer. 


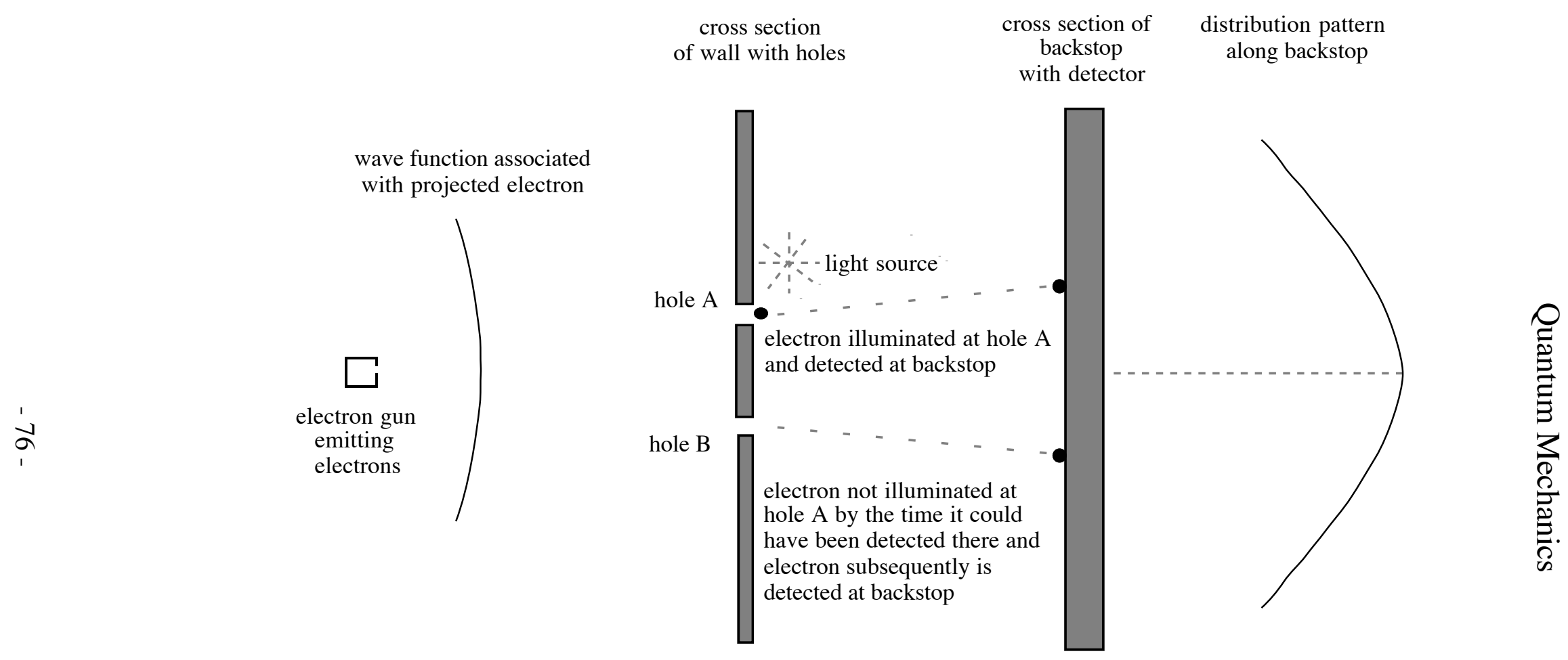

Figure 19

Two-hole gedankenexperiment with strong light source illuminating only one hole.

(Gedankenexperiment 3) 


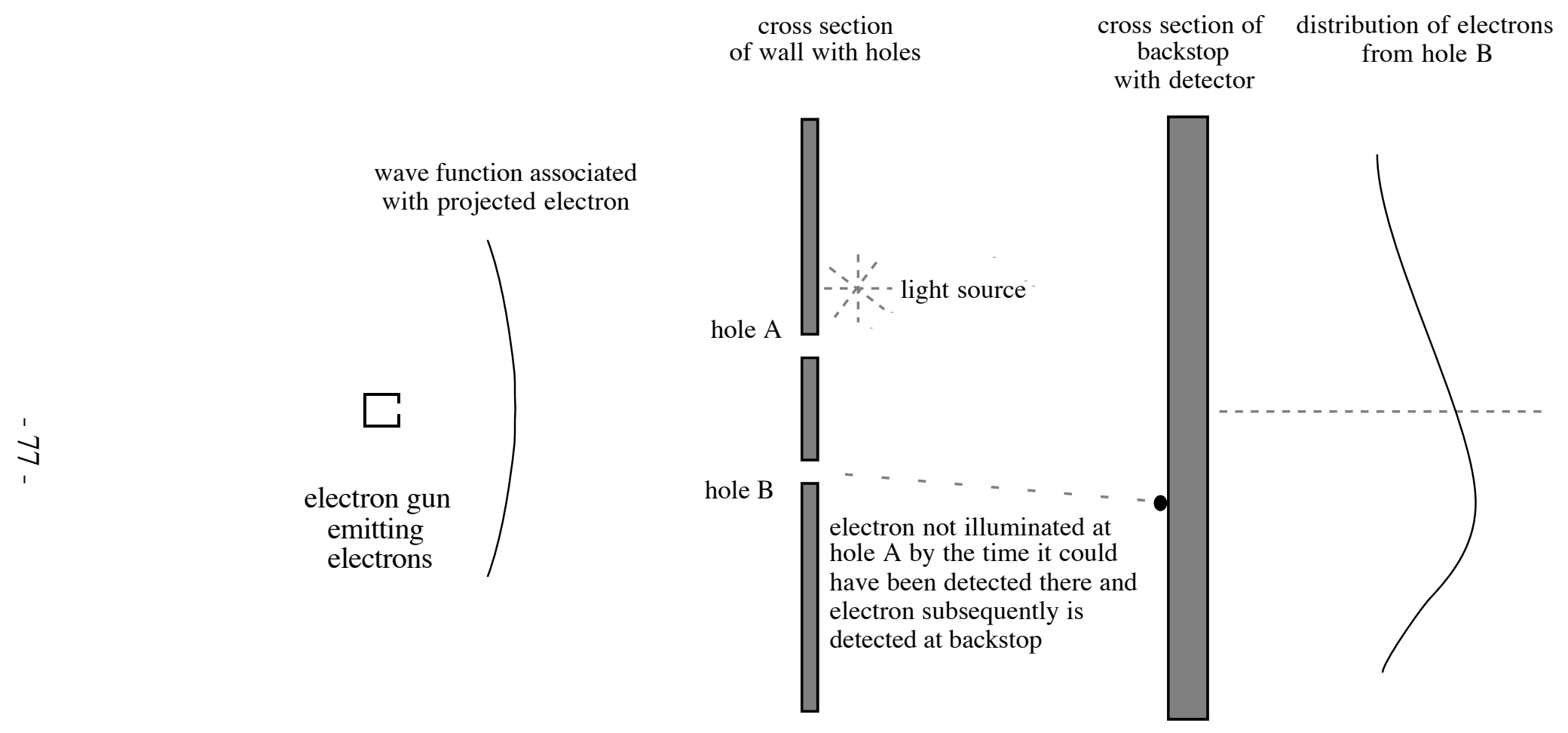

Figure 20

Two-hole gedankenexperiment with strong light source illuminating only one hole in which the distribution of electrons from unilluminated hole is shown. 


\section{Quantum Mechanics}

Epstein (1945) maintained that these kinds of different effects on the physical world in quantum mechanics that cannot be ascribed to physical causes are associated with "mental certainty" (p. 134) on the part of an observer as to which of the possible alternatives for a physical existent occurs. Indeed, the factor responsible for the change in the wave function for an electron headed for holes $\mathrm{A}$ and $\mathrm{B}$, and which is not illuminated at hole $\mathrm{A}$, is knowledge by the observer as to whether there is sufficient time for an electron to pass through the "illuminated" hole. To borrow a term used by Renninger (1960), when the time has elapsed in which the electron could be illuminated at hole A, and it is not illuminated, the observer makes a "negative" (p. 418) observation.

The common factor associated with the electron's passage through the wall in a manner resembling that found for classical-like particles in Gedankenexperiments 2 and 3 is the observing, thinking individual's knowledge as to whether an electron passed through a particular hole. The physical interaction between photons from the light source and electrons passing through either hole 1 or hole 2 is not a common factor. It should be remembered that some causative factor is implied by the very different electron distributions in Gedankenexperiments 1 and 2. It is reasonable to conclude that knowledge by the observer regarding the particular path of the electron through the wall is a factor in the change in the distribution of the electrons in Gedankenexperiment 1 to that found for electrons in Gedankenexperiments 2 and 3.

It might be argued that in Gedankenexperiment 3 a non-human recording instrument might record whether or not an electron passed through the illuminated hole in the time allowed, apparently obviating the need for a human observer. But, as has been shown, a non-human recording instrument is not necessary to obtain the results in Gedankenexperiment 3. And yet even if a non-human instrument is used, ultimately a person is involved to read the results who could still be responsible for the obtained results. Furthermore, one would still have to explain the destruction of the interference affecting the distribution of the electrons at the backstop without relying on a physical interaction between the electrons and some other physical existent. Without ultimately relying on a human observer, this would be difficult to accomplish when the non-human recording instrument presumably relies on physical interactions for its functioning.

It should also be emphasized that the change in the wave function for an electron passing through the unilluminated hole in Gedankenexperiment 3 provides the general case concerning what is necessary for the change in a wave 


\section{Chapter 5}

function to occur in a measurement of the physical existent with which it is associated. It was shown clearly in the extension of Feynman et al.'s gedankenexperiments that the change in the wave function of an electron or other physical existent is not due fundamentally to a physical cause. Instead, the change in the wave function is linked to the knowledge attained by the observer of the circumstances affecting the physical existent measured.

There is one other point to be emphasized. The change in the wave function discussed in Gedankenexperiment 3 serves only to capture the role of knowledge in negative observation. That is, one need not even present a discussion of the wave function to attain the result that knowledge is a factor in the change in the electron distribution in Gedankenexperiment 1 to the electron distribution in Gedankenexperiments 2 and 3. This result depends only on the analysis of experimental results concerning the electron distributions in these three gedankenexperiments.

\section{The Schrödinger Cat \\ Gedankenexperiment}

The nature of the change in the wave function that generally occurs in a measurement will now be discussed in more detail in terms of a gedankenexperiment proposed in 1935 by Schrödinger. In his gedankenexperiment, Schrödinger focused on the immediate change in the wave function that occurs upon observation of a measuring apparatus that records the value of a quantum mechanical quantity.

A cat is penned up in a steel chamber, along with the following diabolical device (which must be secured against direct interference by the cat): in a Geiger counter there is a tiny bit of radioactive substance, so small, that perhaps in the course of one hour one of the atoms decays, but also, with equal probability, perhaps none; if it happens, the counter tube discharges and through a relay releases a hammer which shatters a small flask of hydrocyanic acid. If one has left this entire system to itself for an hour, one would say that the cat still lives if meanwhile no atom has decayed. The first atomic decay would have poisoned it. The $\Psi$-function of the entire system would express this by having in it the living and the dead cat (pardon the expression) mixed or smeared out in equal parts. 


\section{Quantum Mechanics}

It is typical of these cases [of which the foregoing example is one] that an indeterminancy originally restricted to the atomic domain becomes transformed into macroscopic indeterminancy, which can then be resolved by direct observation. (Schrödinger $1935 / 1983$, p. 157)

How does the gedankenexperiment indicate that the nature of the wave function as a link between cognition and the physical world is warranted? It does so in terms of the features of the quantum mechanical wave function cited earlier, one being that there is no source of information concerning the physical world in quantum mechanics other than the probabilistic predictions that yield knowledge of the physical world, predictions that have been supported by empirical test. The second is that these probabilities in general change immediately throughout space upon observation of a quantity of the physical existent that is described by the wave function which is the basis for the probabilistic predictions. Importantly, the velocity limitation of the special theory precludes a physical existent from mediating this change in the wave function.

Note that Schrödinger does not specify how close the observer needs to be to the cat to resolve the indeterminancy. The observer can, in principle, be at any distance from the cat, even across the universe, and initiate this immediate change in the wave function, so long as the observer makes an observation regarding whether the cat is alive. Indeed, the observer does not even have to observe the cat directly but can rely on another observer who has observed the cat and who tells the former observer the result of his observation.

In a related vein, Schrödinger did not explicitly discuss the role and significance of the person as observer in the measurement process in quantum mechanics. Physicists often use the term "observation" ambiguously. Changing the latter part of Schrödinger's quote to indicate that the concern specifically is with a person making the observation does not lessen the statement's validity:

It is typical of these cases [of which the foregoing example is one] that an indeterminancy originally restricted to the atomic domain becomes transformed into macroscopic indeterminancy, which can then be resolved by direct [human] observation.

Thus, in a circumstance where the observer is specified to be a person, the change in the wave function is tied explicitly to the perception by the human 


\section{Chapter 5}

observer of the cat. This point is not limited to those circumstances where a human observer is explicitly specified. This point holds in the general case where a non-human macroscopic measuring instrument intervenes between a quantum mechanical entity and a human observer. It is a human observer who ultimately records the result of any observation. In the cat gedankenexperiment, for example, the cat acts as a macroscopic measuring instrument and comes to be characterized by the same probabilities as the microscopic physical phenomenon (i.e., the radioactive substance) until a human observer makes his own observation of the cat regarding its being alive or dead.

It should be remembered that the Schrödinger cat gedankenexperiment portrays the special case where a macroscopic measuring instrument is used to make a measurement. As has been shown, Gedankenexperiment 3 discussed above provides the general case concerning what is necessary for the change in a wave function to occur in a measurement of the physical existent with which it is associated. There it was also shown that the change in the wave function is linked to the knowledge attained by the observer of the circumstances affecting the physical existent measured and that the change in this wave function is not due fundamentally to a physical cause.

Knowledge and the Measurement of the Spin Component of Electrons Along a Spatial Axis

It has been shown in gedankenexperiments using the two-hole interference scenario of Feynman, Leighton, and Sands that physical interaction is not necessary to effect the change in the wave function that generally occurs in measurement in quantum mechanics. Instead, the general case is that knowledge is linked to the change in the wave function. Another demonstration of this point follows. The models for gedankenexperiments employing electrons (spin one-half particles) presented now are found in Feynman, Leighton, and Sands's (1965) chapter on spin-one particles in their Lectures on Physics. Similar to the earlier gedankenexperiments, these gedankenexperiments also employ negative observation. But in contrast to the earlier gedankenexperiments, readily quantifiable results of the negative observations are developed. In addition, the significance of knowledge to the change of the wave function is emphasized because a concurrent physical interaction to the negative observation between the existent measured and the measuring instrument is shown to be incapable of effecting the change in the wave function. 


\section{Quantum Mechanics}

\section{Basic Features of the Experimental Design}

Consider the case of a device like a Stern-Gerlach type apparatus (device A) which has an inhomogeneous magnetic field where the field direction and the direction of the gradient are the same, for example along the $z$ axis (Figure 21). An electron can pass along one of two paths as it moves through the apparatus. $^{10}$ This is due to the quantization of the spin angular momentum of the electron, more specifically the quantization of the spin component along any spatial axis into two possible values.

Initially, let an electron be in a state such that the probabilities of its going through either of the paths are equal. Which of the two possible paths an electron has passed through depends on whether the electron's spin component along the axis of the inhomogeneous magnetic field of the device is either in, or against, the direction of the magnetic field and its gradient. Given the initial probabilities, one-half of the electrons exiting from device A will be observed to have spin up (i.e., in the direction of the magnetic field and gradient of device A), and one-half of the electrons exiting device A will be observed to have spin down (i.e., opposite to the direction of the magnetic field and gradient of device A). If, after an observation is made, the electron is now put through another Stern-Gerlach type device (device C), identical in construction to the first and oriented in the same direction, the electron will exit along the same path that it exited from in the first machine. In order to do this, the electron must first be brought back to its original direction of motion.

This is accomplished through the use of another Stern-Gerlach type device (device B), the spatial orientation of which is up-down and right-left reversed with respect to the first device. In device $B$, the magnetic field and the gradient are in the opposite direction along the same spatial axis to that found for device A. The placement of these two devices is shown in Figure 22, with devices $\mathrm{A}$ and $\mathrm{B}$ right next to each other. ${ }^{11}$

\footnotetext{
${ }^{10}$ An electron is a member of a class of particles known as fermions. The spin component of a fermion along any spatial axis has two possible values when it is measured: $+1 / 2(h / 2 \pi)$ (spin up along this axis) and $-1 / 2(h / 2 \pi)$ (spin down along this axis). The results of the gedankenexperiment hold for fermions in general.

${ }^{11}$ Note that no pathways are shown in Figure 22 for the electrons traveling through device $\mathrm{AB}$. This is because quantum mechanics provides the correct description of the electrons, and it indicates that an electron does not travel over one or the other of the paths until an observation of the electron is made regarding which path it traveled. Instead, the wave function associated with an electron indicates that the probability is $1 / 2$ that it will have spin up along the $z$ axis and the probability is $1 / 2$ that it will have spin down along the $z$ axis
} 


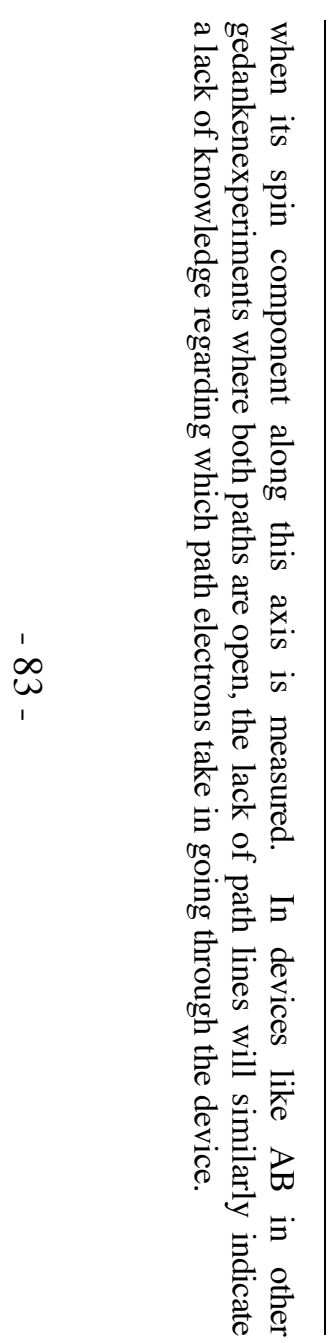

For electrons traveling

toward Device A:

$P_{z}$ spin up $=1 / 2$

$P_{z}$ spin down $=1 / 2$

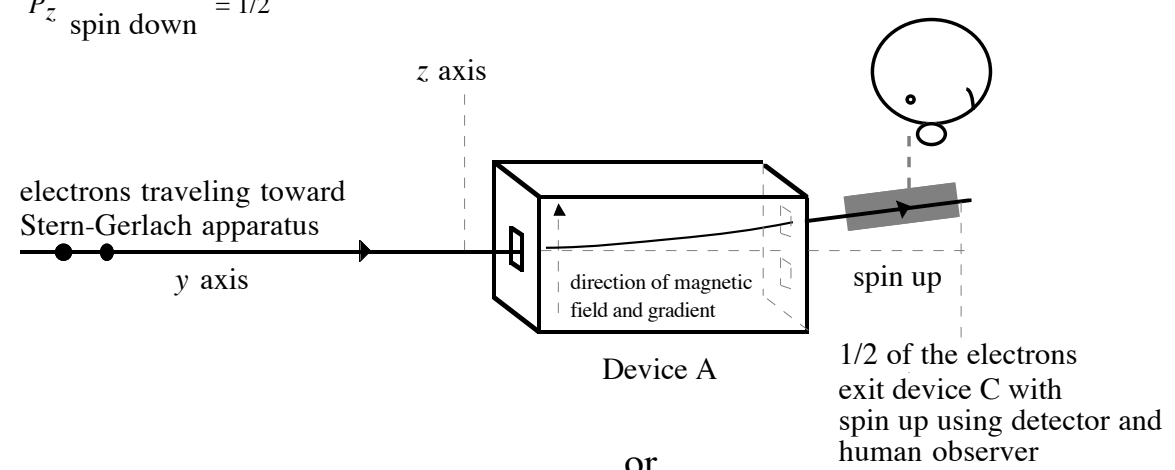

or

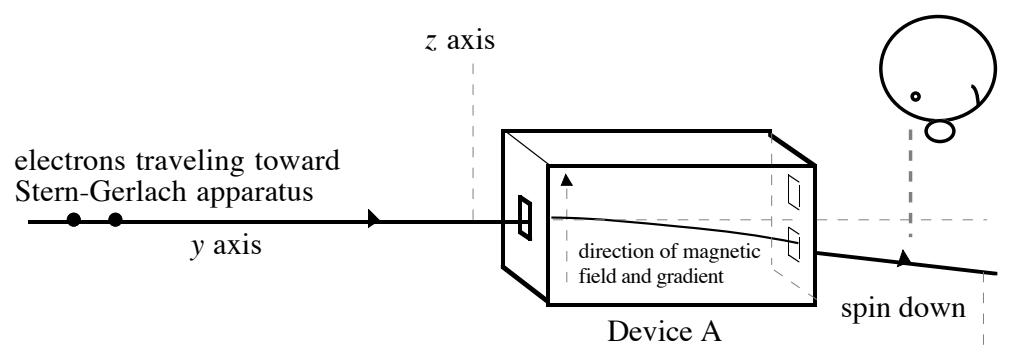

Figure 21

Electrons passing through a Stern Gerlach device.
$1 / 2$ of the electrons exit device $\mathrm{C}$ with spin down using detector and human observer 
For electrons traveling toward Device A:

$P_{z \text { spin up }}=1 / 2$

$P_{z \text { spin down }}=1 / 2$
For electrons traveling

toward Device C:

$P_{z}$ spin up $=1 / 2$

$P_{z \text { spin down }}=1 / 2$

$z$ axis

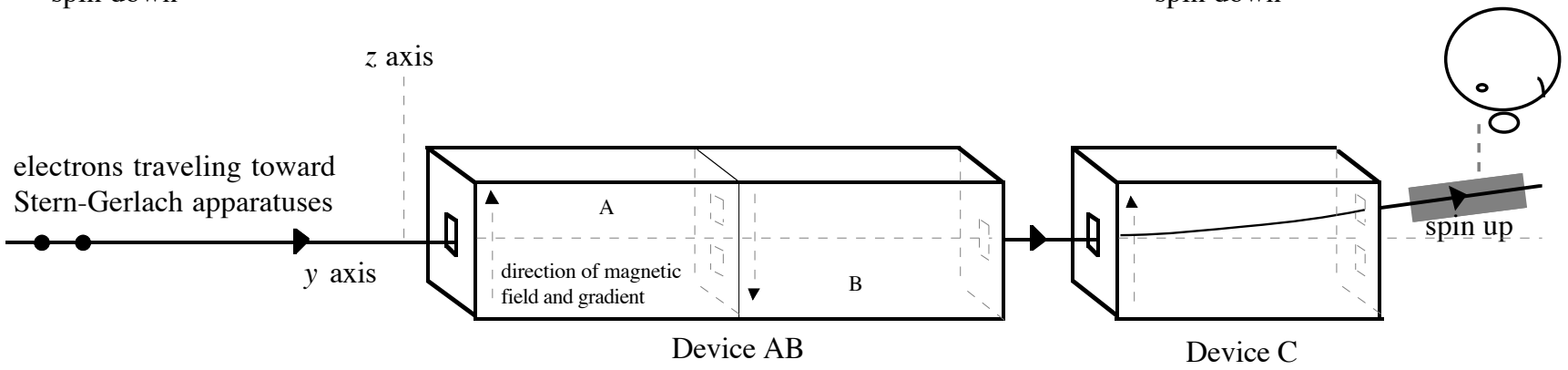

or

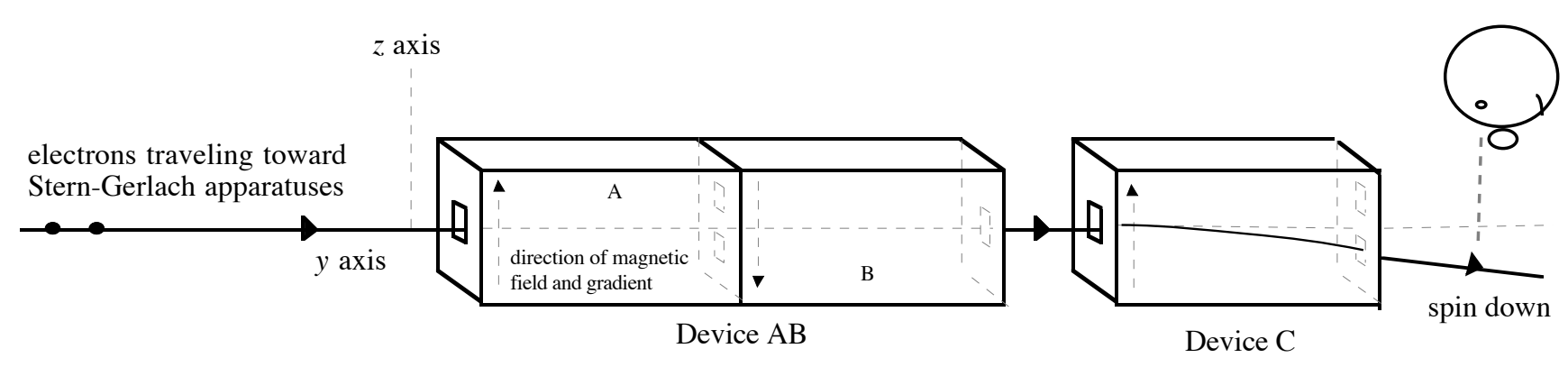

Figure 22

Electrons passing through a series of Stern-Gerlach devices oriented along the same spatial axis $z$. 


\section{Chapter 5}

\section{Two Gedankenexperiments}

Consider the following gedankenexperiments that adhere to quantum mechanical principles and that are supported by empirical evidence. They show that it is an individual's knowledge of the physical world that is tied to the functioning of the physical world itself.

\section{Gedankenexperiment 4}

Allow that device $\mathrm{AB}$ has a block inserted in it as portrayed in Figure 23. Then device $\mathrm{AB}$ allows only electrons with a spin up component along the $z$ axis to exit it. Electrons with a spin down component along this axis are blocked from exiting. Allow that $R$ electrons exit the device with a spin up component. Next to device $\mathrm{AB}$ a second device, $\mathrm{DE}$, is placed that is identical in construction. D is the Stern-Gerlach-like device closest to B. The device DE is tilted around the $y$ axis relative to device AB. $a R$ electrons exit device DE with spin up (where $0<a<1$ ). (Spin up here is relative to the $z^{\prime}$ axis and is in the direction of the magnetic field and gradient of device D.) Next to device DE is device $\mathrm{C}$ in the same spatial orientation as device $\mathrm{A}$ of $\mathrm{AB}$ and its magnetic field and gradient in the same direction along the $z$ axis as device A. A block is inserted into device $\mathrm{C}$ that precludes electrons with spin down from exiting it. $b a R$ electrons exit device $C$ with spin up (where $0<b<1$ ). (Spin up here is relative to the $z$ axis.) (Figure 24 displays the number of electrons exiting the various devices in this and succeeding gedankenexperiments.)

\section{Gedankenexperiment 5}

The experimental arrangement is the same as that in Gedankenexperiment 4, except that no block is inserted in device DE (Figure 25). The numbers of electrons coming out of each device are as follows: (1) $R$ electrons exit device $\mathrm{AB}$ with spin up along the $z$ axis; (2) $R$ electrons exit device DE; and (3) $R$ electrons exit device $C$ with spin up along the $z$ axis.

\section{Discussion of Gedankenexperiments 4 and 5}

How can one account for the results of Gedankenexperiments 4 and 5 ? An observer finds that $R$ electrons exit device $\mathrm{C}$ in Gedankenexperiment 5 , in accordance with the expectation that the spin components of the electrons along the $z$ axis remain unaffected by the passage of the electrons through device DE. It appears that device DE, which has no block, has no effect on the spin components along the $z$ axis of the electrons passing through it. $R$ electrons exit device A with spin up along the $z$ axis and $R$ electrons exit device $\mathrm{C}$ with 


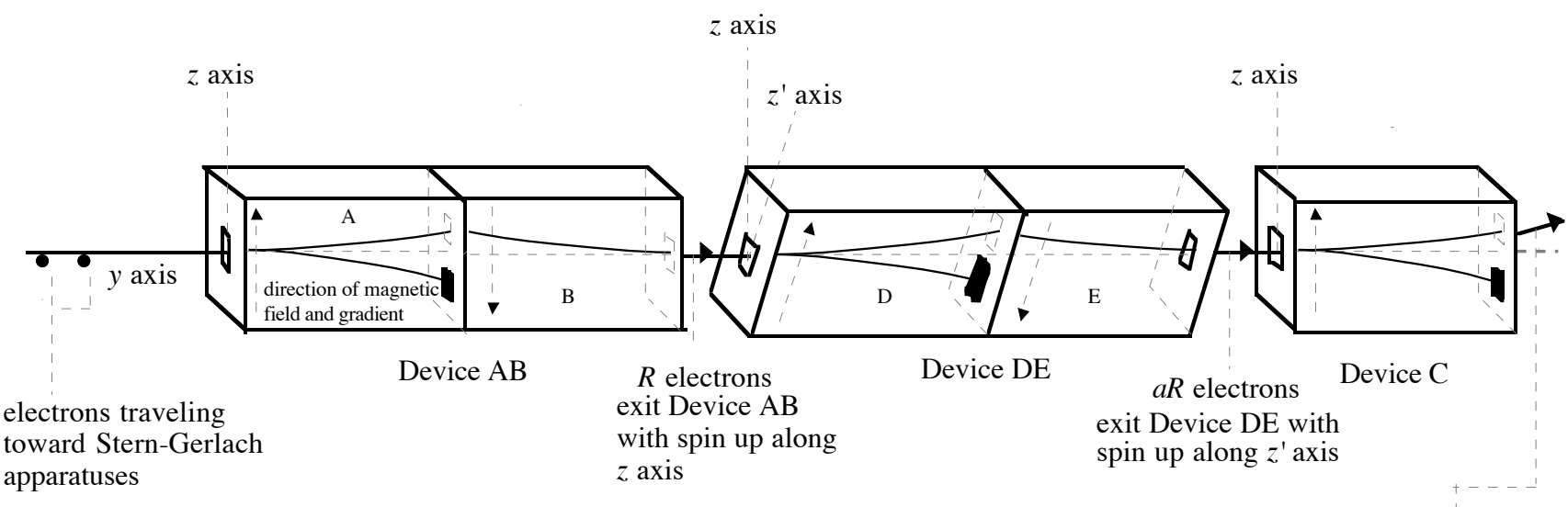

baR electrons

Figure 23

exit Device $C$ with spin up along $z$ axis

A series of Stern-Gerlach devices where only electrons with spin up along the $z$ axis pass through device $\mathrm{AB}$, only electrons with spin up along the $z^{\prime}$ axis pass through device $\mathrm{DE}$, and only electrons with spin up along the $z$ axis pass through device C. (Gedankenexperiment 4) 


\begin{tabular}{|c|c|c|c|c|c|c|c|c|c|}
\hline \multirow{2}{*}{$\begin{array}{l}\text { Gedanken- } \\
\text { experiment }\end{array}$} & \multicolumn{3}{|c|}{ Device $A B$} & \multicolumn{3}{|c|}{ Device DE } & \multicolumn{3}{|c|}{ Device C } \\
\hline & $\begin{array}{l}\text { Paths } \\
\text { Open }\end{array}$ & $\begin{array}{l}\text { Orientation } \\
\text { of Magnetic } \\
\text { Field }\end{array}$ & $\begin{array}{l}\text { Number of } \\
\text { Electrons } \\
\text { Exiting AB }\end{array}$ & $\begin{array}{l}\text { Paths } \\
\text { Open }\end{array}$ & $\begin{array}{l}\text { Orientation } \\
\text { of Magnetic } \\
\text { Field }\end{array}$ & $\begin{array}{l}\text { Number of } \\
\text { Electrons } \\
\text { Exiting DE }\end{array}$ & $\begin{array}{l}\text { Paths } \\
\text { Open }\end{array}$ & $\begin{array}{l}\text { Orientation } \\
\text { of Magnetic } \\
\text { Field }\end{array}$ & $\begin{array}{l}\text { Number of } \\
\text { Electrons } \\
\text { Exiting } \\
\mathrm{C}\end{array}$ \\
\hline 4 & spin up & $z$ axis & $R$ & spin up & $z^{\prime}$ axis & $\alpha R$ & spin up & $z$ axis & $b \alpha R$ \\
\hline 5 & spin up & $z$ axis & $R$ & $\begin{array}{l}\text { spin up } \\
\text { and spin } \\
\text { down }\end{array}$ & $z^{\prime}$ axis & $R$ & spin up & $z$ axis & $R$ \\
\hline 6 & spin up & $z$ axis & $R$ & $\begin{array}{l}\text { spin } \\
\text { down }\end{array}$ & $z^{\prime}$ axis & $\nu R$ & $\begin{array}{l}\text { spin } \\
\text { down }\end{array}$ & $z$ axis & $u v R$ \\
\hline 7 & spin up & $z$ axis & $R$ & $\begin{array}{l}\text { spin up } \\
\text { and spin } \\
\text { down }\end{array}$ & $z^{\prime}$ axis & $R$ & $\begin{array}{l}\text { spin } \\
\text { down }\end{array}$ & $z$ axis & 0 \\
\hline
\end{tabular}

Figure 24

Specifications and results for Gedankenexperiments 4 through 7. 


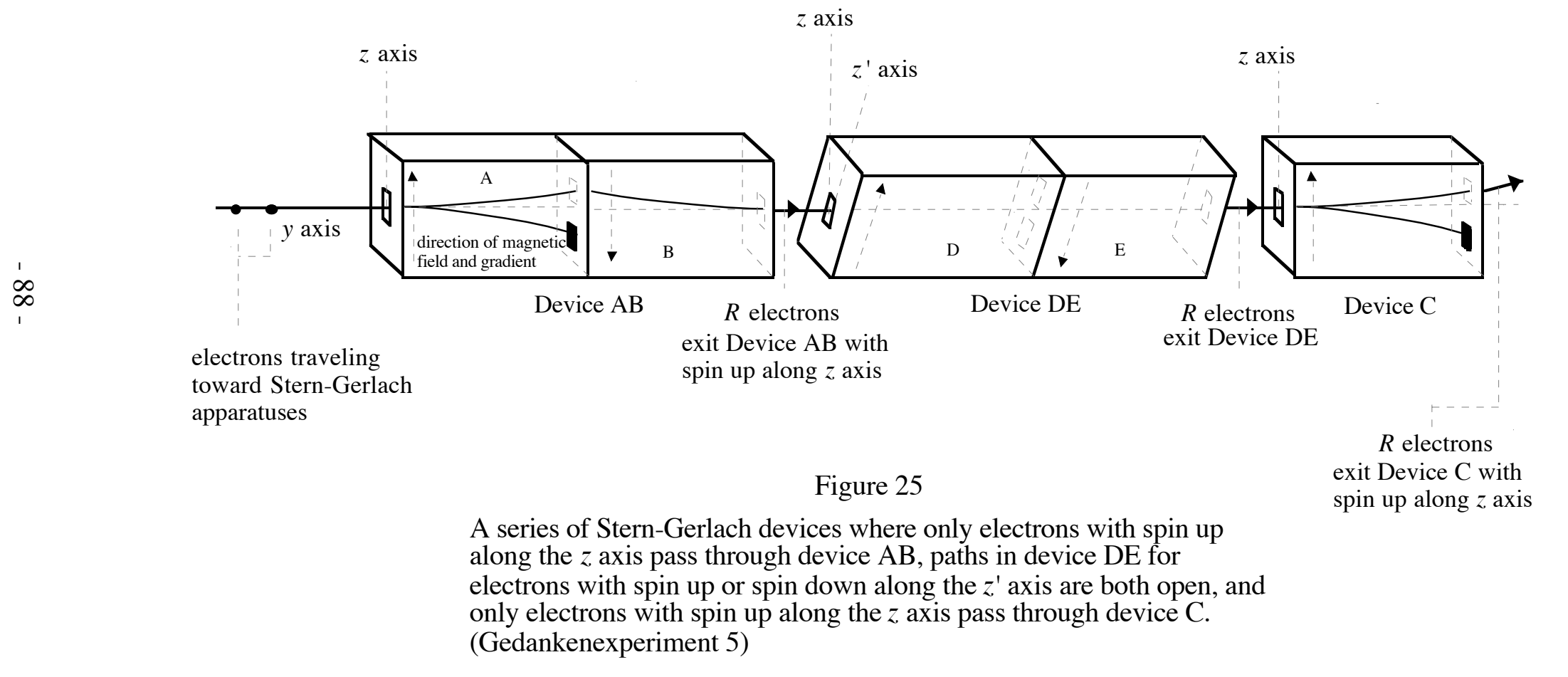

A series of Stern-Gerlach devices where only electrons with spin up (Gedankenexperiment 5) 


\section{Chapter 5}

spin up along the $z$ axis. All electrons pass through device DE. But Gedankenexperiment 4 does not provide a similar result. A similar result would be that $a R$ electrons would exit device $\mathrm{C}$ in Gedankenexperiment 4, not baR electrons. That is, the spin components of the electrons along the $z$ axis would essentially remain unaffected by the passage of the electrons through device $\mathrm{DE}$ in Gedankenexperiment 4, just as device DE in Gedankenexperiment 5 does not appear to affect the spin components of electrons along the $z$ axis. How is it that $b a R$ electrons exit from device $\mathrm{C}$ in Gedankenexperiment 4 instead of $a R$ electrons? It is reasonable to conclude that something unusual is happening to the electrons in their passage through device DE in Gedankenexperiment 4, particularly in view of the results of Gedankenexperiment 5. Somehow the spin components of the electrons along the $z$ axis are affected by their passage through device DE in Gedankenexperiment 4 while device DE in Gedankenexperiment 5 does not affect the spin components of electrons along the $z$ axis.

A comparison of Gedankenexperiments 4 and 5 indicates that the only physical feature of the measuring apparatus that can possibly be responsible for the change in the component of the spin angular momentum along the $z$ axis of the electron is the block that is inserted in device DE in Gedankenexperiment 4. Other than this one difference, the measuring apparatuses in Gedankenexperiments 4 and 5 are identical.

\section{The Block in Device DE}

The experimental consequences resulting from the presence or absence of the block in device DE in Gedankenexperiments 4 and 5 concern whether one or both paths are open in device DE. Significantly, it is electrons traveling along the unblocked path in Gedankenexperiment 1 that exhibit the unusual behavior regarding the frequency of electrons exiting device $C$. Thus, the nature of the effect of the influence of the block on the electrons is indeed unusual from a conventional standpoint, a standpoint that would expect the change in spin components along the $z$ axis of the electrons that travel along the unblocked path to somehow be changed by a physical interaction with the block. This physical interaction, though, is not possible. The scenario involving a block is thus in essence a negative observation. A negative observation occurs where an observation is made by deducing that a particular physical event must have occurred because another physical event did not occur with subsequent consequences for the functioning of the physical world stemming from the change in knowledge. Physical interaction as the basis for the consequences in the physical world is ruled out. Remember that the spin 


\section{Quantum Mechanics}

components of the electrons along the $z$ axis traveling through device DE are affected by the change in knowledge, as evidenced by $b a R$ electrons exiting device $\mathrm{C}$ in Gedankenexperiment 4 instead of $a R$ electrons. As previously noted, empirical work on electron shelving that supports the existence of negative observation has been conducted by Nagourney, Sandberg, and Dehmelt (1986), Bergquist, Hulet, Itano, and Wineland (1986), and by Sauter, Neuhauser, Blatt, and Toschek (1986).

\section{A Variation of the Gedankenexperiments}

Two other gedankenexperiments similar to Gedankenexperiments 4 and 5 will provide an even more remarkable demonstration that an individual's knowledge of the physical world is tied to the functioning of the physical world itself.

\section{Gedankenexperiment 6}

The experimental arrangement is the same as that in Gedankenexperiment 4, except that the blocks are inserted in devices DE and C such that spin up electrons along $z$ and $z$, respectively, cannot exit these devices and spin down electrons are allowed to proceed unimpeded (Figure 26). The numbers of electrons coming out of each device are as follows: (1) $R$ electrons exit device $\mathrm{AB}$ with spin up along the $z$ axis; (2) $v R$ electrons exit device DE with spin down along the $z^{\prime}$ axis; and (3) $u v R$ electrons exit device $C$ with spin down along the $z$ axis.

\section{Gedankenexperiment 7}

The experimental arrangement is the same as that in Gedankenexperiment 6, except that device DE has both paths open (Figure 27). The numbers of electrons coming out of each device are as follows: (1) $R$ electrons exit device $\mathrm{AB}$ with spin up along the $z$ axis; (2) $R$ electrons exit device DE; and (3) 0 electrons exit device $C$ with spin down along the $z$ axis.

\section{Discussion of Gedankenexperiments 6 and 7}

The result in Gedankenexperiment 6 is remarkable. How is it that electrons with spin down along the axis of the magnetic field of the measuring device $\mathrm{A}$, oriented in a particular direction along $z$, are found exiting device $\mathrm{C}$, in which the axis of its magnetic field and its gradient are also oriented in the same direction along $z$ ? No electron with spin down along the $z$ axis exits device AB. This result is particularly unusual when in Gedankenexperiment 7, 


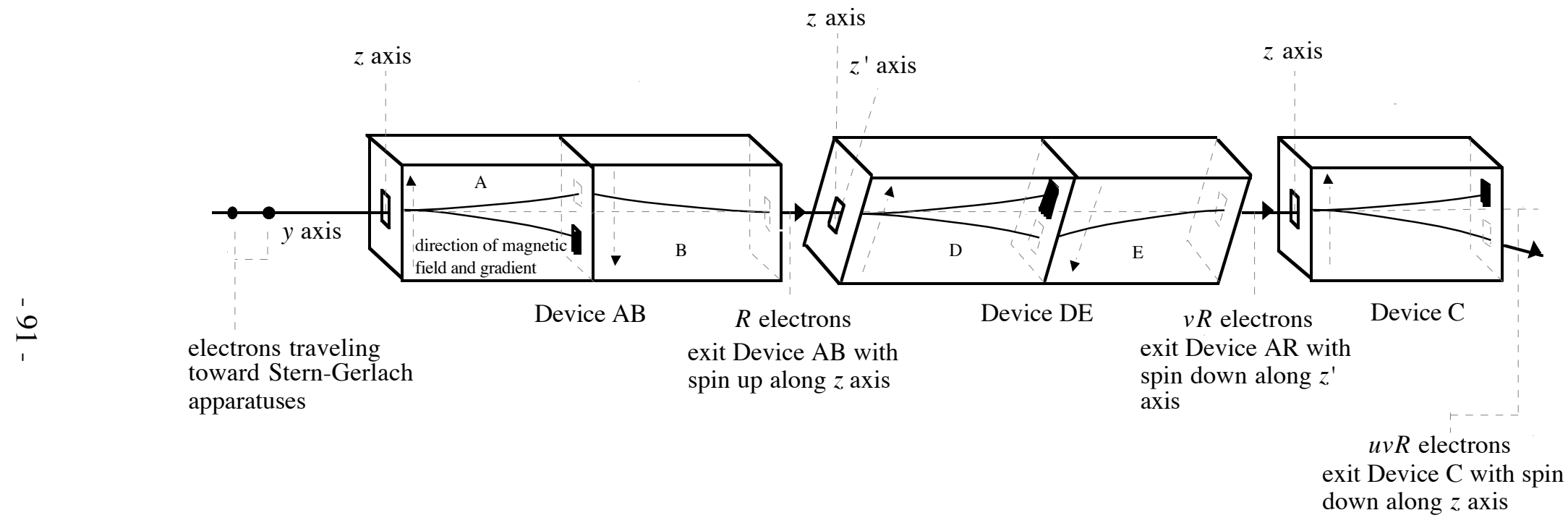

Figure 26

A series of Stern-Gerlach devices where only electrons with spin up along the $z$ axis pass through device $\mathrm{AB}$, only electrons with spin down along the $z$ axis pass through device $\mathrm{DE}$, and only electrons with spin down along the $z$ axis pass through device $C$. (Gedankenexperiment 6 ) 
$z$ axis

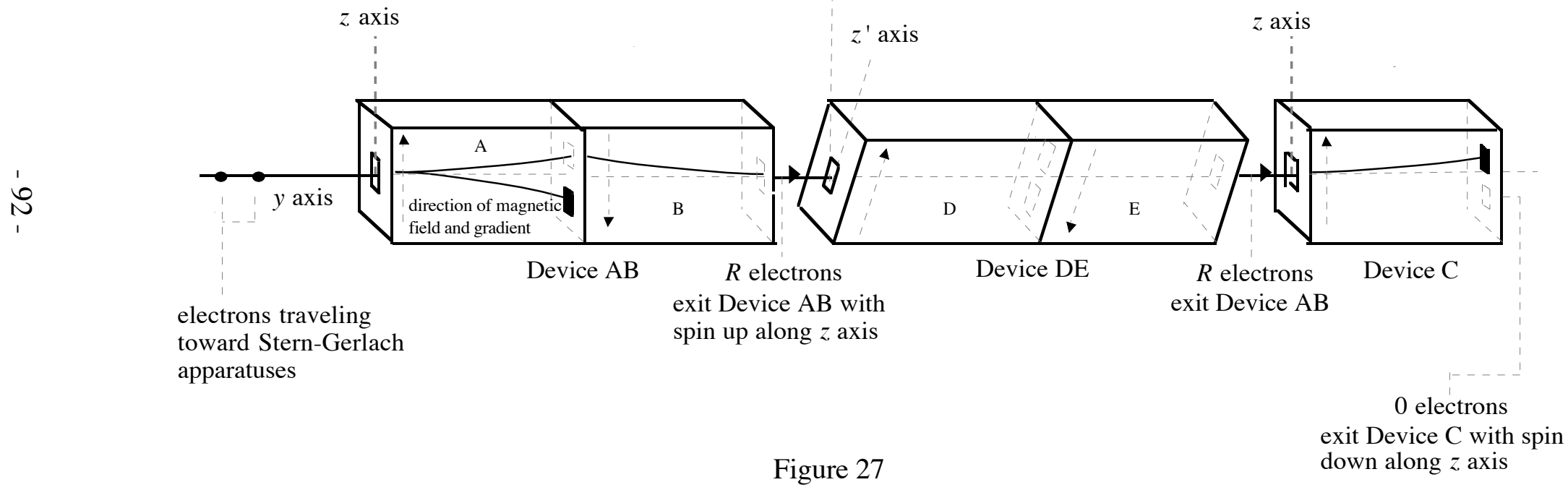

A series of Stern-Gerlach devices where only electrons with spin up along the $z$ axis pass through device $\mathrm{AB}$, paths in device $\mathrm{DE}$ for electrons with spin up or spin down along the $z$ ' axis are open, and only electrons with spin down along the $z$ axis pass through device $\mathrm{C}$. (Gedankenexperiment 7) 


\section{Chapter 5}

using the same device DE, modified only by the removal of the block that prevents electrons with a spin up component along $z^{\prime}$ (the axis of the magnetic field in DE) to pass, there are no electrons that exit device $\mathrm{C}$ with spin down along the axis of its magnetic field, which has the same spatial orientation as the magnetic field of A along the $z$ axis.

In Gedankenexperiment 7, it appears as if the spin components of the electrons along the $z$ axis were not affected by their passage through device DE, which has both paths open and which thus allowed all electrons to pass through. As reflected in the behavior of the electrons that pass through device $\mathrm{C}$, the spin components of the electrons along the $z$ axis in Gedankenexperiment 6 are affected by device DE, specifically by the insertion of the block in this device that prevents electrons with spin up components along the $z^{\prime}$ axis from exiting device DE. Again, no electrons with spin down along this axis were found to exit device $\mathrm{AB}$. The electrons traveling along the unblocked path in device DE in Gedankenexperiment 6 exhibit this unusual behavior regarding the frequency of electrons exiting device $C$. No physical interaction between the block in device DE and any electron traveling along the unblocked path is responsible for the frequency of electrons exiting device C. In Gedankenexperiment 6 , a negative observation at device $\mathrm{DE}$ has resulted in electrons exiting device $\mathrm{C}$ with spin down along the $z$ axis whereas in the absence of a negative observation, in Gedankenexperiment 7, no electrons exit device $\mathrm{C}$ with spin down along the $z$ axis.

\section{Interference}

The difference in the observer's knowledge of the spin components of electrons along an axis, and the difference in the spin components of the electrons themselves, in the pairs of gedankenexperiments that have been presented (i.e., Gedankenexperiments 4 and 5, and 6 and 7) reflect the presence or absence of interference in the wave functions associated with each of the electrons. For example, in terms of the formalism, in Gedankenexperiment 4 the probability amplitude $a_{1}$ for an electron exiting device $\mathrm{AB}$ with spin up $(A B+)$ and exiting device $\mathrm{C}$ with spin up $(C+)$ is given by

$$
a_{1}=<C+|D E+><D E+| A B+>\text {. }
$$

The probability of these events is derived by taking the absolute square of this probability amplitude, $\left|a_{1}\right|^{2}$. In contrast, in Gedankenexperiment 5 , the probability amplitude $d$ for an electron exiting device $\mathrm{AB}$ with spin up $(A B+)$ and exiting device $\mathrm{C}$ with spin up $(C+)$ is given by 


\section{Quantum Mechanics}

$$
d=<C+|D E-><D E-| A B+>+<C+|D E+><D E+| A B+>.
$$

When the absolute square of the probability amplitude $d$ is calculated to yield the probability that an electron exiting device $A B$ with spin up will exit device $C$ with spin up, it is evident that there will be two terms representing interference. These terms are

$$
(<C+|D E-><D E-| A B+>)^{*}(<C+|D E+><D E+| A B+>)
$$

and

$$
(<C+|D E+><D E+| A B+>)^{*}(<C+|D E-><D E-| A B+>) .
$$

It is these terms that distinguish $|d|^{2}$, where there is interference, from $\Sigma|a|_{i}^{2}$ where one knows which path the electron took through device DE and there is no interference

$$
\Sigma\left|a_{i}\right|^{2}=\left|a_{1}\right|^{2}+\left|a_{2}\right|^{2}
$$

or

$$
\sum|a|_{i}^{2}=|<C+| D E+><D E+|A B+>|^{2}+|<C+| D E-><D E-|A B+>|^{2} .
$$

It is important to emphasize that it is not the presence or absence of the block in device DE that interacts with electrons that is responsible for the presence or absence of interference in Gedankenexperiments 4 and 6 . It is the act of knowing the value of the spin component of the electron along the $z^{\prime}$ axis that is responsible. The block in device DE in Gedankenexperiment 4 and the block in device DE in Gedankenexperiment 6 serve as bases for negative observations.

\section{Another Indication of the Importance of Knowledge}

in Measurement in Quantum Mechanics

There is one more feature of the gedankenexperiments discussed in this paper that supports the theses that: 1) the macroscopic nature of a physical apparatus used for a measuring instrument is not central to making a measurement in quantum mechanics; 2) knowledge is central to making such measurements; and 3) the role of the block in device DE in Gedankenexperiments 4 and 6 is to provide information. Gedankenexperiments 4 through 7 demonstrate the interesting point that the magnetic field of device DE itself is not sufficient to induce the change in the wave function that device DE which has a block along one path does for electrons traveling along the unblocked path and with which the block does not physically interact. Unless 


\section{Chapter 5}

there is some way in the physical set up of device DE to determine the spin component of the electron along an axis $z^{\prime}$ (as is done in Gedankenexperiments 4 and 6 by the block in device DE), there is no change in the wave function of the electron concerning its spin components.

In Gedankenexperiment 5 where there is no possibility in the physical set up that is device DE to know the spin component of the electron in device DE (because a block is not inserted along either the "spin up" or the "spin down" path), device DE does not affect the spin components along the $z$ axis of the electrons as they travel through. That is, the number of electrons exiting devices $\mathrm{C}$ and $\mathrm{AB}$ are exactly the same. Also, in gedankenexperiment 7, no electrons with spin down along the $z$ axis exit device $\mathrm{C}$ and only electrons with spin up along the $z$ axis exit device $\mathrm{AB}$. Without a block in device DE in Gedankenexperiment 7, there is no change in the wave function of an electron as regards its spin components. This is equivalent to saying that there has been no measurement of the spin component along the $z^{\prime}$ axis of the electron. To quote Feynman et al. (1965) regarding their filtering experiments with spin-one particles similar in principle to Gedankenexperiments 4 and 6:

The past information [concerning spin along the $z$ axis after exiting the first device] is not lost by the separation into...beams [in the second device], but by the blocking masks that are put in [the second device] (p. 5-9).

In conclusion, if an interaction between a macroscopic physical apparatus and the existent to be measured were responsible for a change in the wave function of the physical existent measured, why, if a magnetic field by itself is unable to effect this change in the wave function for electrons, is the insertion of a block able to effect this change for electrons traveling through the unblocked path? When device DE does not contain a block along one of the paths, electrons traveling along what is the unblocked path in Gedankenexperiment 4 or the unblocked path in Gedankenexperiment 6 do not undergo any change in their wave function. The role of the block in Gedankenexperiments 4 and 6 is to provide information to a human observer concerning electrons traveling along the unblocked path. With regard to these electrons, the role of the block in the measurement of their spin components along the $z^{\prime}$ axis does not depend on a physical interaction between them and the block. 


\section{Quantum Mechanics}

\section{The Time of a Measurement}

The question is often asked concerning quantum mechanics how can an observer finding out about a measurement that has presumably been made some time earlier be linked to the measurement itself? In terms of Gedankenexperiment 4 , for example, if a human observer finds out about the electrons passing through the devices $\mathrm{AB}, \mathrm{DE}$, and $\mathrm{C}$ only after the electrons exit device $\mathrm{C}$, how can this observer be considered responsible in some way for a measurement that was presumably made at device DE because of the inclusion of the block in that device? That is, a negative observation seems to be made only after the electrons exit device $\mathrm{C}$, even though the block in device $\mathrm{DE}$ made the information available earlier (i.e., as soon as the time elapsed in which an electron passing through device DE could reach the block at the end of D).

The analysis underlying the question presumes that some form of physical interaction occurring within a temporal framework provides the basis for measurement in quantum mechanics even though it clearly does not. In Gedankenexperiment 4, this presumed physical interaction does not occur in device DE. Measurement in quantum mechanics is fundamentally concerned with the development of knowledge. The course of physical interactions over time is not the central factor in the development of this knowledge. It is knowledge that is primary and within this knowledge, the functioning of the physical world, including the course of physical interactions over time, occurs.

As has been discussed, there are other indications for this view concerning the importance of knowledge in quantum mechanics. Knowledge of the physical world is developed using wave functions, and wave functions provide only probabilistic knowledge. The quantum mechanical wave function associated with a physical existent generally changes immediately throughout space upon measurement of the physical existent. This change in the wave function is not limited by the velocity limitation of the special theory of relativity for physical existents, the velocity of light in vacuum. There is the complex number nature of the wave function from which information concerning the physical world is derived.

The Effect of Measurement on the Past

One other point provides support for the central significance of knowledge in measurement in quantum mechanics. In Gedankenexperiments 4 and 6 , the presence of the block, or more accurately the knowledge that results from the presence of the block, at the exit of device D affects the electrons 


\section{Chapter 5}

traveling along the unblocked path in device D from their entry into device D for two reasons:

1. If the block is removed prior to the end of the time over which an electron could traverse device D along the blocked path, interference would not be destroyed and the number of electrons exiting device $\mathrm{C}$ in Gedankenexperiment 4 (i.e., with spin up along the $z$ axis), for example, is the same as the number of electrons exiting device $\mathrm{AB}$.

2. With the block in place and the time elapsed over which an electron could have reached the block in device $\mathrm{D}$, the interference that was supposed to characterize the electron in its passage through device $\mathrm{D}$ did not occur as the electron could have traveled along only the unblocked path. If a detector had been set up along any part of the path in device D containing the block prior to the electron's having reached the end of device $\mathrm{D}$ where the block is situated, the electron would not have been detected along the path containing the block.

A negative observation that the block allows for by providing information to an observer is thus seen to affect one's knowledge of the past as well as the past itself, in the present case indicating that the electron has traveled down a particular path in device $\mathrm{D}$ as opposed to being characterized by a wave function demonstrating interference and not having traveled one path exclusively.

\section{The Third Component}

The probabilistic basis of knowledge in quantum mechanics indicates that cognition is directly linked to the physical world. In statistical mechanics, this connection is even more clear, because the basic principles of statistical mechanics are statistical in nature. Unlike quantum mechanics, these principles are not modeled on processes in the physical world. They do not involve physical law in any deterministic way. The application of this fundamentally statistical model to the physical world, and its empirical support, demonstrate in a beautiful way the link between cognition and the physical world. 


\section{Chapter 6}

\section{Statistical Mechanics}

Finally, the third component of modern physical theory, statistical mechanics, requires exploration. In statistical mechanics, probabilistic knowledge of the physical world is developed from statistical principles without recourse to other more fundamental underpinnings. Specifically, this probabilistic knowledge is developed without modeling the principles of statistical mechanics on lawful processes of the physical world. This probabilistic knowledge is linked to the physical world because the knowledge depends fundamentally on rational processes and yet provides the correct description of physical processes. Unlike quantum mechanics which is concerned with individual physical existents, statistical mechanics is concerned with large physical systems with many components.

In physics, there is an incongruity between physical law that is characterized by order, and randomness, that is at the heart of statistical mechanics. The foundations of modern physical law, that is quantum theory and the special and general theories of relativity, have been discussed in previous chapters. Quantum theory and relativity theory in their own way supplanted what had been for over two hundred years prior to their development the basis of physical law, namely Newtonian mechanics. Though there are very significant differences between quantum theory, relativity theory, and Newtonian mechanics, they share a very important characteristic. Each theory, in its own way, prescribes some sort of completely ordered development of variables related to quantities in the physical world. ${ }^{12}$ It is this completely ordered development of variables concerning physical quantities that entitles these theories to be considered the basis for physical law.

We have seen that quantum theory and relativity theory both incorporate features that indicate that mind and the physical world are linked. Statistical mechanics also indicates such a link. Unlike quantum theory, statistical mechanics is at base solely probabilistic in nature, and the values of any quantities of a physical system that are explained in statistical mechanics arise only out of probabilistic considerations once certain parameters of the physical situation are specified. The probabilistic knowledge attained through the use of

\footnotetext{
${ }^{12}$ In quantum mechanics, the ordered development is concerned with the wave function that provides the basis for probabilistic predictions.
} 


\section{Statistical Mechanics}

statistical mechanics is developed independently of the physical world once the initial situational parameters are given. The principles at the core of statistical mechanics are very simple and yet provide for very powerful predictions, both in scope and specificity, concerning physical phenomena. Regarding classical thermodynamics, the fundamental explanation of which lies in statistical mechanics, Einstein wrote:

It [classical thermodynamics] is the only physical theory of universal content concerning which I am convinced that, within the framework of applicability of its basic concepts, it will never be overthrown. (Kittel, 1969, p. 1)

\section{Basic Principles of Statistical Mechanics}

Three basic principles of statistical mechanics are tied to what is known as the fundamental assumption. These principles are:

1) the existence of a closed (or isolated) physical system composed of a number of entities, for example particles;

2) an ensemble of physical systems all constructed like the actual closed physical system of interest, except that each system is in exactly one of the accessible stationary quantum states for the closed physical system of interest;

3) the fundamental assumption itself, namely that it is equally likely that a closed system will be in any accessible stationary quantum state interest (Kittel, 1969).

Figure 28 depicts a representative ensemble like that noted in principle 2 for four small magnets that can only be oriented in one of two directions along an axis in space.

In large part, the chapter is concerned with the demonstration that the core of statistical mechanics is not fundamentally dependent on the physical world for its development and instead depends fundamentally on rational processes. Indeed, the fundamental assumption in principle 3 is reasonably made in the absence of a direction from the physical world regarding how to consider the accessible stationary quantum states of a closed physical system.

\section{An Isolated Physical System}

An isolated, or closed, physical system in statistical mechanics is defined as having constant energy and volume and a constant number of 
4 up, 0 down

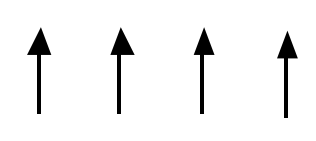

3 up, 1 down
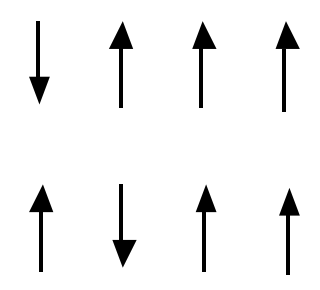

$\uparrow \uparrow \downarrow \uparrow$

$\uparrow \uparrow \uparrow$
2 up, 2 down

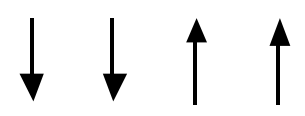

$\downarrow \uparrow \downarrow \uparrow$
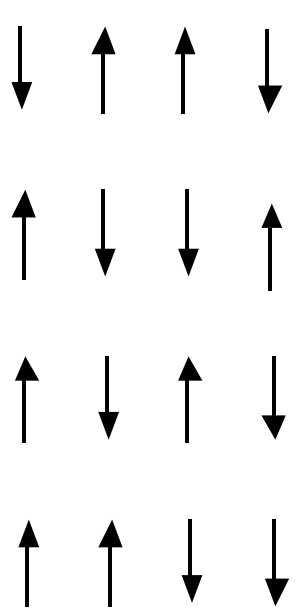

1 up, 3 down
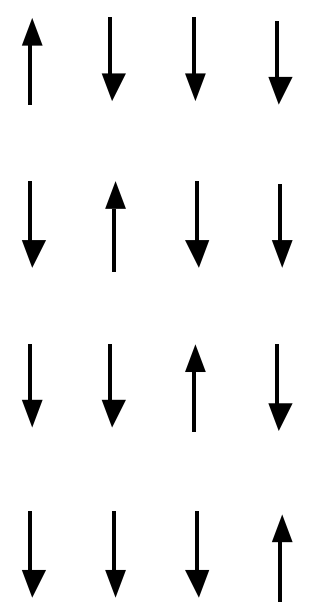

0 up, 4 down

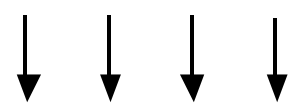

Figure 28

Accessible states in representative ensemble $\left(2^{\mathrm{N}}=16\right)$ for 4 small magnets $(\mathrm{N}=4)$ with regard to magnetic moment either "up" or "down." Arrows represent the magnets in each ensemble, with the direction of the magnetic moment of a magnet indicated by the direction of the arrow. 


\section{Statistical Mechanics}

particles. When all observable quantities of a physical system, including its energy, are independent of time, the system is said to be in a stationary state. An accessible stationary state of this system is one in which the energy of the system is very similar to the actual energy and the number of particles is equal to the number of particles of the actual system. One can see in Figure 28 that the magnets can assume either of two directions along a spatial axis, and this latitude accounts for the different accessible stationary states of the magnets. Applying the fundamental assumption to the system of magnets, one finds that the state of the system is randomly determined.

In statistical mechanics, a primary concern is the number of accessible stationary quantum states available to a physical system. The natural logarithm of this number is the entropy of the system. ${ }^{13}$ Kittel (1969) wrote:

The entropy is the most important quantity of thermal physics [statistical mechanics]: from the entropy we find the temperature, the pressure, the chemical potential, the magnetic moment, and the other functions of thermal physics. (p. 3)

For an isolated system of magnets, not subject to an external force, like that shown in Figure 28, the distribution of the accessible stationary states with regard to the number of magnets having magnetic moment "up" is binomial. It can be shown that for a system as small as 100 magnets, the binomial distribution for this system closely resembles a normal distribution. This can be seen in comparing Figures 29 and 30.

\section{Entropy and Thermal Equilibrium}

One of the most important areas of predictions concerns the temperatures of two physical systems that are placed in thermal but not diffusive contact (i.e., they are allowed to exchange energy but not particles) and that together form an isolated physical system. The second law of thermodynamics allows one to predict that these two component systems will tend toward thermal equilibrium (i.e., toward having the same temperature) over time when

\footnotetext{
${ }^{13}$ Feynman, Leighton, and Sands (1963) wrote that entropy is the logarithm of "the number of ways that the insides [of a specified physical system] can be arranged, so that from the outside it looks the same" (p. 7-46).
} 


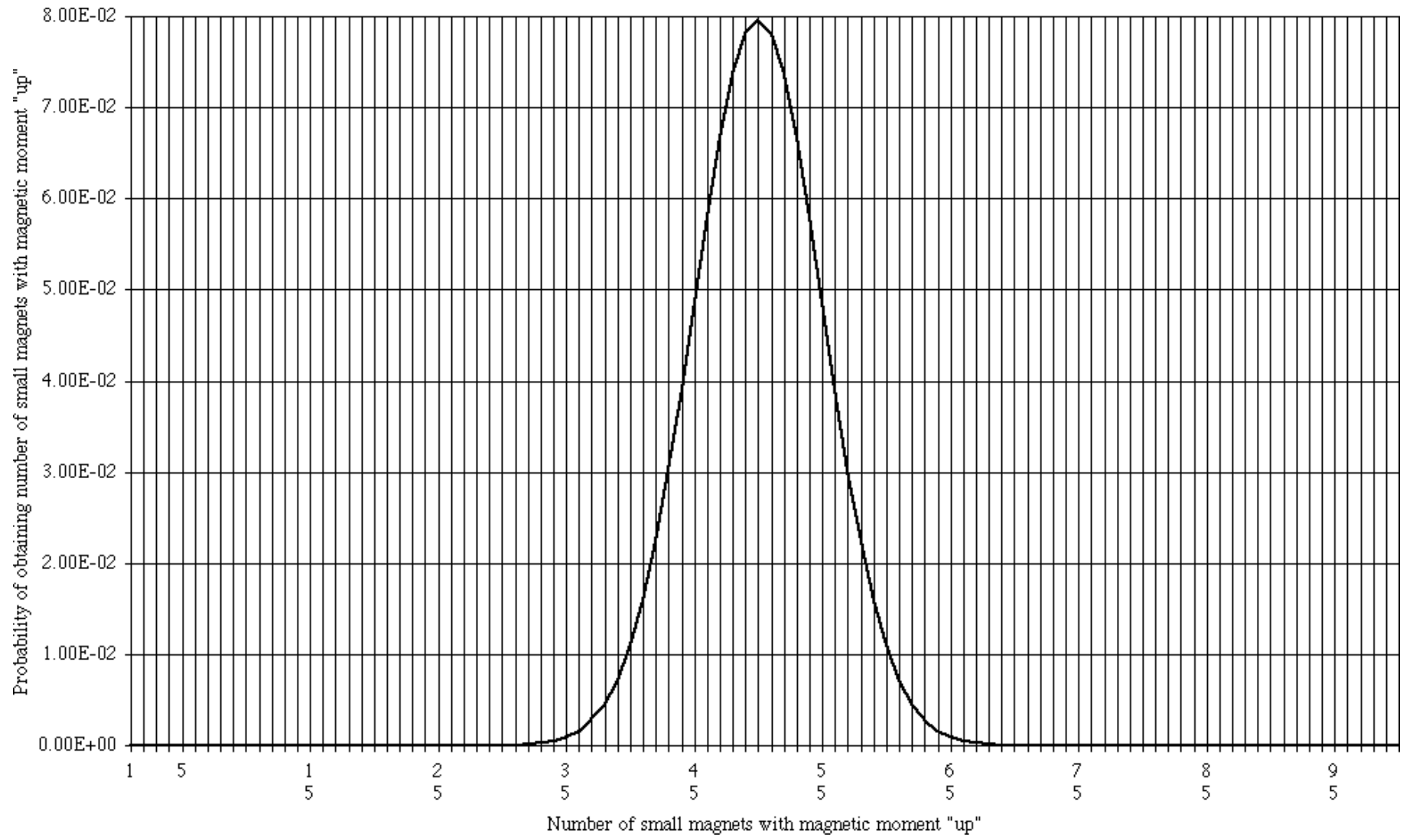

Binomial distribution of 100 small magnets with regard to magnetic moment "up." 


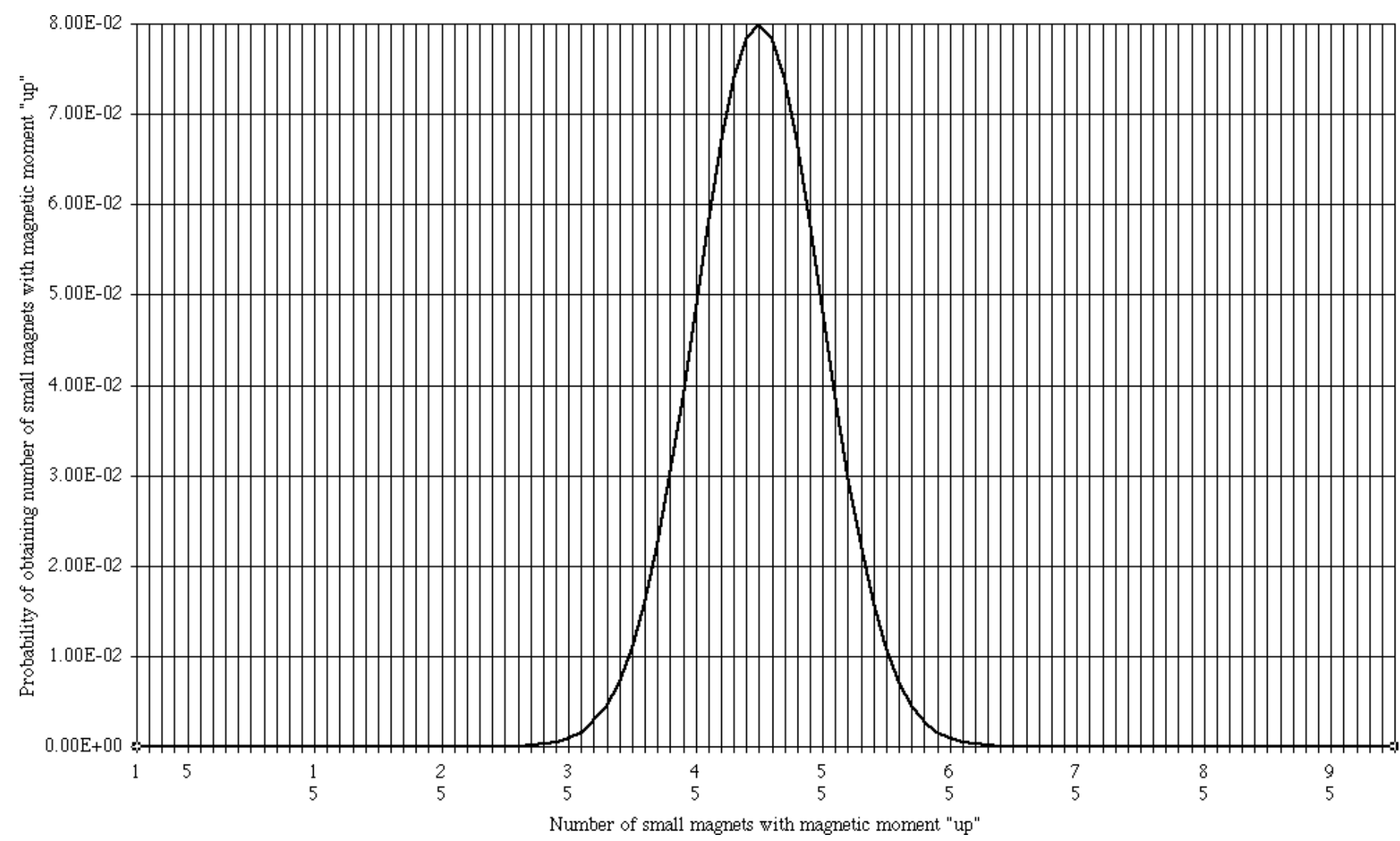

Normal distribution function applied to 100 small magnets with regard to magnetic moment "up." 


\section{Chapter 6}

they are brought into thermal contact. ${ }^{14}$ Why do the two component systems act in this way? The answer lies in the fundamental assumption's particular application to the overall, isolated physical system. If the overall, isolated physical system has a particular energy, this energy may be divided between the two component systems in many ways. The energy of the overall system is divided between the component systems so as to maximize the number of accessible states of the overall system. The normal distribution for each of the component systems considered as isolated physical systems sets up the number of accessible states of each of the component systems at different energy levels for each of the component systems.

\section{An Example of the Tendency to Thermal Equilibrium}

Consider an example involving an isolated physical system composed of many small magnets that can only be oriented along one spatial axis in space. This system could be represented by a figure similar to Figure 31. If a uniform magnetic field is introduced to the system, there would be a number of different energy levels of the overall system of magnets depending on the excess magnetic moment for the overall system (i.e., the difference of the number of magnets with magnetic moment "down" along the spatial axis from the number of magnets with magnetic moment "up"). The number of accessible states for the physical system would be associated with its particular energy level in the presence of the uniform external magnetic field. For example, in a system of 100 magnets, there are 100 states where 1 magnet has spin "up" and 99 magnets have spin "down." For this same system, there are $100 \times 10^{27}$ states where 50 magnets have spin "up" and 50 magnets have spin "down." Each of the particular combinations of spin "up" and spin "down" is a configuration.

Allow that two systems of small magnets are in a uniform magnetic field (and thus have specific energies associated with them) and that these systems are brought into thermal contact, but not diffusive contact, to form a large physical system. There would then be a particular energy for the overall physical system. The two systems would tend toward thermal equilibrium, that is they would tend toward a configuration that contains the largest number of accessible states for the overall system. This tendency is a direct consequence of the fundamental assumption. This configuration, called the most probable

\footnotetext{
${ }^{14}$ That the second law of thermodynamics is called a law is a bit problematic because it is built on randomness even though on the macroscopic level it describes the physical world with great accuracy.
} 


\section{Statistical Mechanics}

configuration, has a specific excess magnetic moment for each of the component systems and thus implies a particular energy for each of the component systems. Nothing in the physical world prevents the overall system from implementing this direct consequence of the fundamental assumption, except that the energy of the component systems must be distributed to accommodate for the achievement of the most probable configuration.

It should be noted that the temperature of a physical system thus depends not only on the energy but the entropy, the degree of disorder, of this system. (Please see Appendix E for the mathematical representation of temperature and thermal equilibrium.) Two physical systems in thermal equilibrium have the same temperatures when the change in entropy in one of the component systems that occurs as a result of a small change in the energy of this system is balanced by an opposite change in the entropy that occurs in the other component system as a result of the corresponding change in energy of this other system. As the change in energy in one system must be balanced by an opposite change in the energy of the other system (because the energy of the overall system is constant), so too must a change in the entropy of one system be balanced by an opposite change in the other system. Thus, thermal equilibrium is achieved when the number of accessible states cannot increase. Concerning the role of probability in linking the energy and temperature of a physical system, and by implication the importance of entropy to temperature, Planck wrote:

The general connection between energy and temperature may only be established by probability considerations. [Two systems] are in statistical equilibrium when a transfer of energy does not increase the probability. (Kittel, 1969, p. 37)

The three fundamental principles, and the normal distribution which they lead to, have been shown to provide the basis for understanding how interacting physical systems approach thermal equilibrium when they combine to form a large isolated system. The adoption of the fundamental assumption and the consequences deduced from its adoption are rational in nature and have no essential tie to the physical world other than that they provide for correct predictions of physical quantities.

These principles underlie the operation of heat engines and refrigerators, physical systems in thermal contact with their respective environments. They also underlie predictions concerning the chemical potential of physical systems 


\section{Chapter 6}

in diffusive, as well as thermal, contact. In diffusive contact, physical systems can exchange particles as well as energy. Chemical potential governs the movement of particles between systems in diffusive and thermal contact. Chemical potential plays a significant role in processes as varied as the transmission of neural impulses to the operation of electrochemical batteries. As Kittel noted, entropy is a significant factor in the chemical potential of a physical system.

\section{The Significance of \\ the Fundamental Assumption}

In The Principles of Statistical Mechanics, Tolman (1938) stated the basis for adopting the fundamental assumption in statistical mechanics. ${ }^{15}$ Tolman wrote:

In the first place, it is to be emphasized, in accordance with the viewpoint here chosen, that the proposed methods are to be regarded as really statistical in character, and that the results which they provide are to be regarded as true on the average for the systems in an appropriately chosen ensemble, rather than as necessarily precisely true in any individual case. In the second place, it is to be emphasized that the representative ensembles chosen as appropriate are to be constructed with the help of an hypothesis, as to equal a priori probabilities [of each of the accessible stationary quantum states of the large physical system], which is introduced at the start, without proof, as a necessary postulate....It is to be appreciated that some postulate as to the a priori probabilities...has in any case to be chosen. This again is merely a consequence of our commitment to statistical methods. It is analogous to the necessity of making some preliminary assumption as to the probabilities for heads or tails in order to predict the results to be expected on flipping a coin. In the second place, it is to be emphasized that the actual assumption, of equal a priori probabilities...is the only general hypothesis that can reasonably be chosen....In the absence of any knowledge except that our systems do obey the laws of

\footnotetext{
${ }^{15}$ Kittel (1969) included this text from Tolman (1938) in the first edition of his book, Thermal Physics, on pages 34 and 35.
} 


\section{Statistical Mechanics}

mechanics, it would be arbitrary to make any assumption other than that of equal a priori probabilities. (pp. 63-64)

As has been noted, it is the fundamental assumption that leads to the remarkable property in statistical mechanics that the elements in a physical system tend to occupy the widest variety of states possible. Bennett (1987) illustrated this remarkable property of statistical mechanics that the fundamental assumption leads to in the following gedankenexperiment:

A classic irreversible process, and one that helps in defining the concept of entropy a little more precisely, is called free expansion. Suppose a chamber filled with gas is separated by a partition from a vacuum chamber of the same size. If a small hole is made in the partition, gas will escape (that is, it will expand freely) into the formerly empty chamber until both chambers are filled equally.

The reason the molecules spread out to fill both chambers is mathematical rather than physical, if such a distinction can be made. The numbers of molecules on the two sides of the partition tend to equalize not because the molecules repel one another and move as far apart as possible, but rather because their many collisions with the walls of the container and with one another tend to distribute them randomly throughout the available space, until about half of them are one side of the partition and about half are on the other side.

Since the spreading of the molecules is due to chance rather than to repulsion [italics added], there is a chance that all the molecules might return simultaneously to the chamber from which they came. If there are $n$ molecules, however, the probability of all of them returning to their original chamber is the same as the probability of tossing $n$ coins and having them all come up "heads": $1 / 2^{n}$. Thus for any sizable number of molecules (and there are about 300,000,000,000,000,000,000,000 molecules in a gram of hydrogen) the free expansion is an effectively irreversible process: a process whose spontaneous undoing, although possible, is so unlikely that one can say with confidence that it will never be observed. 


\section{Chapter 6}

The disordered state--the state in which the gas has spread into both chambers rather than residing compactly in a single chamber--is more probable than the ordered state. That is, there are more configurations of molecules in which the molecules occupy both chambers, just as, when 100 coins are tossed, there are more ways to achieve a total of 50 heads and 50 tails than there are to achieve 100 heads and no tails [italics added]. In saying that the entropy of the universe tends to increase, the second law is simply noting that the universe tends to fall into more probable states as time passes. (p. 110)

In principle, physical law has nothing to do with the tendency of the gas molecules to distribute throughout the enlarged chamber as opposed to their congregating in one of the chambers. One might think that this tendency to distribute throughout the enlarged chamber is only a gradually increasing tendency to disorder that is a reflection of the physical law governing the interaction of the gas molecules at work. This thesis, though, does not allow for the effect of the doubling of the number of paths that the molecules can travel when the chamber is enlarged. The tendency for the molecules to distribute uniformly reflects a relationship between the many gas molecules considered as a system, and this relationship is not determined by physical law. Instead, like Bennett noted, the relationship between the many gas molecules is mathematical, or statistical.

Though Bennett was correct in distinguishing the spreading of the molecules due to random events rather than a repulsive force that is physical in origin, it is important to note that the force associated with the chemical potential which is largely responsible for the spreading of the molecules in Bennett's scenario is no less a physical force than a force that is physical in origin (i.e., due to a law of nature). As Kittel and Kroemer (1969/1980) wrote:

The chemical potential is equivalent to a true potential energy: the difference in chemical potential between two systems is equal to the potential barrier [due to physical law] that will bring the two systems into diffusive equilibrium [in which the chemical potentials of the two systems are equal]. (p. 124) 


\section{Statistical Mechanics}

\section{More on the Fundamental Assumption}

Contrast individual interactions between two moving particles with a physical system composed of many moving and distinguishable particles, such as that found in a gas. In statistical mechanics, the motion of these particles is considered anything but ordered. Their motion is considered random in nature with the components of this motion along three orthogonal spatial axes considered random as well.

At the same time, it has not been doubted that individual components of a large physical system composed of many distinguishable particles, such as a gas, each follow the laws of nature. But it is not the lawful behavior of individual physical existents that leads to the second law of thermodynamics. Rather, it is the complexity attendant on the in principle random behavior of a large collection of such existents. Brewer and Hahn (1984) reviewed the contrast between physical law and the fundamental assumption of statistical mechanics in its historical context.

In 1872 Ludwig Boltzmann, a founder of modern thermodynamics, gave a lecture in which he said that the entropy, or disorder, of isolated systems increases irreversibly as time passes. On hearing this the physicist Joseph Loschmidt rose in protest. He argued that the laws governing the motions of all particles are symmetric with respect to time. Thus any system that had decayed from order to chaos could be made orderly once again simply by reversing the momentum of each particle, without affecting the total kinetic energy of the system. In defiance Boltzmann pointed his finger at Loschmidt and said, "You reverse the momenta."

This scholarly conflict illustrates the paradoxical nature of the second law of thermodynamics, which states that systems tend toward maximum entropy. Yet Loschmidt's argument remains cogent. If one were able to film the motions of any small group of particles and show the film to a physicist, he or she would have no way of telling in principle whether the projector was running forward or backward. Consequently, according to Loschmidt's criticism (which has come to be called the Loschmidt paradox), any law that governs the behavior of 


\section{Chapter 6}

large collections of particles should be symmetric with respect to time. (p. 50)

It is to be emphasized that physical law does not prescribe the behavior of large collections of physical existents when they are considered in terms of their aggregate behavior. That these physical existents should operate in a disordered manner is not due to physical law; rather it is chance. The problem with considering the large system as simply the sum of the individual components, the behavior of each of which is governed by physical law, is that for the individual components the ways in which the same kind of interactions between the components considered in the aggregate can occur is not at issue. Rather, it is the character of the interactions between the individual components that is of concern. But for the large system considered as a whole, the character of the particular interactions is not the major concern. Rather, it is the ways in which the same interactions between constituents can occur that is of concern. And the ways in which each of the interactions between specified constituents can occur are equally likely. That the individual components of the large system each follow the laws of nature is the basis for Tolman's statement quoted above:

In the absence of any knowledge except that our systems do obey the laws of mechanics, it would be arbitrary to make any assumption other than that of equal a priori probabilities. (Tolman, 1938, p. 64)

It is this reasonable assumption, nonetheless freely made, that allows Tolman (1938) to call statistical mechanics, "really statistical in character" (p. 34). According to Tolman, the order found in the laws of mechanics is necessary to support the thesis that only the fundamental assumption can reasonably be considered to account for the distributions that are the basis of statistical mechanics. Basically, we need to know what physical law is so as to know what it is not. Though the physical interactions among the members of small groups of particles are lawful in nature, the Loschmidt paradox does not affect the validity of the fundamental assumption for isolated, large systems.

The Representative Ensemble

The third principle underlying the development of the normal distribution of various physical quantities concerns a representative ensemble of systems. The physicist's imagination is the basis for this ensemble of physical systems in which the components are similar to the actual system of interest, in particular its energy and number of particles (Kittel, 1969). The use of the 


\section{Statistical Mechanics}

representative ensemble has been verified by experiments supporting the predictions of statistical mechanics. Regarding the representative ensemble, Kittel (1969) wrote in the first edition of Thermal Physics:

Boltzmann and Gibbs made a conceptual advance in the problem of calculating average values of physical quantities. Instead of taking time averages over a single system, they imagined a group of a large number of similar systems, suitably randomized. Averages at a single time are taken over this group of systems. The group of similar systems is called an ensemble of systems. The average is called the ensemble average or the thermal average.

An ensemble is an intellectual construction [italics added] that represents at one time the properties of the actual system as they develop in the course of time....Our assumption is that this ensemble represents the system in the sense that an average over the ensemble gives correctly the value of the average for the system [over time].

The Gibbs scheme replaces time averages over a single system by ensemble averages, which are averages over all systems in an ensemble. The demonstration of the equivalence of the ensemble and time averages is difficult [italics added] and has challenged many mathematicians. The book by Tolman [The Principles of Statistical Mechanics] gives an excellent and readable discussion of the general question. It is certainly plausible that the two averages might be equivalent, but one does not know how to state the necessary and sufficient conditions that they are exactly equivalent [italics added]. (pp. 32-33)

In the above quote, Kittel implied that time averages for one system and averages for a representative ensemble may well be equivalent, even though demonstrating this equivalence has not been accomplished. Kittel's comments in the quote on the equivalence of the two types of averages are interesting because it is not the position taken by Tolman in The Principles of Statistical Mechanics, a book that was clearly important to Kittel in his writing his textbook on the subject. After discussing the conceptual basis of the argument for how the two averages may be considered equivalent, Tolman pointed out limitations in the argument that the time average for a physical system and the 


\title{
Chapter 6
}

ensemble average are equivalent. The limitations indicate that the time average for a physical system cannot be substituted for the ensemble average developed with the representative ensemble. (Please see Appendix F for Tolman's presentation of the argument concerning the equivalence of time and ensemble averages and reasons why the argument is not convincing.) Consequently, the cognition involved in developing the representative ensemble is central to the results achieved in statistical mechanics concerning the physical world, results that are supported by empirical evidence.

It should be noted that in the second edition of Thermal Physics, Kittel and Kroemer (1969/1980) eliminated the above quote from the first edition, removing the reference to time averages and their possible equivalence with ensemble averages. Instead, Kittel and Kroemer referred to the representative ensemble in the following way:

\begin{abstract}
An ensemble of systems is composed of many systems, all constructed alike. Each system in the ensemble is a replica of the actual system in one of the quantum states accessible to the system. If there are $g$ accessible states, then there will be $g$ systems in the ensemble, one system for each state. Each system in the ensemble is equivalent for all practical purposes to the actual system. Each system satisfies all external requirements placed on the original system and in this sense is "just as good" as the actual system. (p. 31)
\end{abstract}

\section{Summary}

In statistical mechanics, the probabilities from which the predictions of statistical mechanics are derived are not modeled after processes of the physical world. It is the absence of suitable processes that make the fundamental assumption from which the probabilities are developed reasonable. Thus, order as expressed in physical law does not explain the tendency of a large system to occupy that configuration allowing for the most accessible stationary quantum states of the system. The predictions in statistical mechanics that are based on probabilistic considerations are concerned with knowledge and are derived on rational grounds. The representative ensemble that is central to the development of predictions concerning physical systems in statistical mechanics is an intellectual construction that is not equivalent to circumstances in the physical world. Yet these predictions of physical quantities have been supported by empirical evidence. 


\section{Statistical Mechanics}

The above points indicate that knowledge in statistical mechanics is not simply a reflection of the functioning of a physical world independent of the knowing individual. These points indicate further that the predictive knowledge in statistical mechanics is linked to the physical world itself. These results are reinforced by the fact that probabilistic predictions characterize not only the behavior of large groups of physical entities in statistical mechanics, but also in quantum mechanics such predictions characterize the behavior of the existents considered individually.

Now that the three major components of modern physical theory have been explored and evidence has been shown to support the thesis that cognition is directly linked to the physical world in each major component, the question arises: can psychology makes its own contribution to exploring the link between mind and the physical world that is found in modern physical theory? 


\section{Chapter 7}

\section{Can Psychology Make Its Own Contribution?}

Analysis of modern physical theory indicates that there is a fundamental link between the observing, thinking individual and the physical world. This being the case, both psychological research methodology and results should be relevant to the exploration of this link. It is important to provide an example of how psychology can add in a unique way to the exploration of the link between cognition and the physical world found in modern physical theory. The following is a prototype for more complicated investigations.

Gedankenexperiments are proposed that indicate that research from psychology is relevant to understanding the relationship between perception and the physical world in quantum mechanics. These gedankenexperiments rely on research in psychology concerning the perceptual and behavioral adaptation of a human observer to inversion of incoming light. The gedankenexperiments presented demonstrate how the uncertainty principle may be affected by psychological phenomena. The uncertainty principle involves the inability in quantum mechanics to simultaneously measure observable quantities described by non-commuting Hermitian operators (e.g., the position and momentum of a particle along a particular spatial axis). ${ }^{16}$ Further, the gedankenexperiments show that it may be possible to know more than one value at a time from a set of what appear to be mutually exclusive values for a quantity.

When light is reflected from an object in the world and into the eye, the structure of the eye is such that the image on the retina corresponding to the object is up-down and right-left reversed when compared to the position of the object (Dolezal, 1982; Kandel, Schwartz, \& Jessell, 1991). The area of psychological research relevant to the proposed gedankenexperiments specifically concerns the effect of inversion of incoming light on visual experience. (In the context of this paper, unless more precisely specified, the terms "inversion" and "inverted" will refer to either rotation of the incoming light $180^{\circ}$ around the line of sight, or the reflection [or flipping] of incoming light between the top and lower halves of the visual field along the horizontal

\footnotetext{
16 Feynman, Leighton, and Sands's (1965) implication, noted in Chapter 5, that physical interaction is responsible for the constraints of the uncertainty principle is not the case.
} 


\section{Chapter 7}

separating them.) First, this research will be reviewed. Then the gedankenexperiments will be described.

$$
\begin{aligned}
& \text { The Effect of Inversion of Incoming Light } \\
& \text { on Visual Perception and Visually Guided Behavior }
\end{aligned}
$$

In the late 1800s, Stratton $(1896,1897 \mathrm{a}, 1897 \mathrm{~b})$ investigated the effects on visual experience of rotating the incoming light $180^{\circ}$ around the line of sight such that the retinal images were right side up instead of being in their customary inverted orientation. Stratton's results were remarkable. In commenting on the earlier experiment, he wrote:

In fact, the difficulty of seeing things upright by means of upright retinal images seems to consist solely in the resistance offered by the long-established experience. There is certainly no peculiar inherent difficulty arising from the new conditions themselves. If no previous experience had been stored up to stand in opposition to the new perceptions, it would be absurd to suppose that the visual perceptions in such a case would seem inverted. Any visual field in which the relations of the seen parts to one another would always correspond to the relations found by touch and muscular movement would give us 'upright' vision, whether the optic image lay upright, inverted, or at any intermediate angle whatever on the retina. (Stratton, 1896, p. 617)

His comments apply as well to the results of the second, more thorough experiment. In his report on the second experiment, he wrote:

The inverted position of the retinal image is, therefore, not essential to 'upright vision,' for it is not essential to a harmony between touch and sight, which, in the final analysis, is the real meaning of upright vision. For some visual objects may be inverted with respect to other visual objects, but the whole system of visual objects can never by itself be either inverted or upright. It could be inverted or upright only with respect to certain non-visual experiences with which I might compare my visual system--in other words, with respect to my tactual or motor perceptions. (Stratton, 1897b, pp. 475-476) 


\section{Can Psychology}

Subsequent work by other researchers in which all incoming light was rotated $180^{\circ}$ around the line of sight (e.g., Ewert, 1930; Snyder \& Pronko, 1952) or up-down reversed (the top and bottom halves of the visual field are reversed) (e.g., Dolezal, 1982; Kohler, 1962, 1964) has for the most part provided substantial support for Stratton's finding concerning the relative nature of upright vision. The research indicates that there is a high degree of flexibility of the visual system with regard to inversion of incoming visual stimulation on the retina, including that an observer subject to such reversal quickly regains very significant competency in interacting with the environment. For example, Snyder and Pronko (1952) found in their study:

During the 30-day period that the inverting lenses were worn, the visuo-motor coordinations were refashioned so that the subject performed even better than before the lenses were put on....Introducing the inverted visual field for 30 days and subsequent "normalization" (lenses removed), [sic] modified the learning situation. However, the subject went on learning despite these disrupting factors (p. 116).

In general, visual experience restabilizes quickly considering the relatively very brief period of time that the light is inverted compared to the subjects' life experiences prior to their participation in one of the experiments. Visual experience regains a sense of normalcy and is accompanied by the coordination between touch and vision that Stratton (1897b) wrote is "the real meaning of upright vision" (p. 497) (Dolezal, 1982; Erismann \& Kohler, 1953, 1958; Kohler, 1962, 1964; Pronko \& Snyder, 1951; Snyder \& Pronko, 1952).

As alluded to in the above quote from Snyder and Pronko and as found by Ewert (1930), in the laboratory, competency on sensorimotor tasks developed with unrotated light has been shown to transfer to circumstances where incoming light is rotated $180^{\circ}$ (Snyder \& Pronko, 1952). Furthermore, increased competency on the same sensorimotor tasks subsequently developed with rotated light has been shown to transfer to circumstances where the incoming light is no longer rotated $180^{\circ}$ (Ewert, 1930; Snyder \& Pronko, 1952). The learning curve for these tasks was in general fairly smooth, except

for a spike when the incoming light was first rotated $180^{\circ}$ around the line of sight. In natural settings, individuals wearing an optical apparatus that inverted incoming light have reported such activities as driving an automobile, riding a motorcycle, or riding a bicycle, with a significant degree of skill within a relatively short time of putting on the apparatus for the first time (Dolezal, 1982; 


\section{Chapter 7}

Erismann \& Kohler, 1953, 1958; Kohler, 1962; Snyder \& Pronko, 1952). In sum, research has indicated that after a relatively brief period of time exposed to inverted light, visual experience in general appears normal and as this visual experience exists in conjunction with the recaptured competency of the individual in the environment, the visual field is upright, just as it was upright before the incoming light was inverted. The citations to Erismann and Kohler (1953, 1958) and to Pronko and Snyder (1951) refer to films that provide convincing evidence of both perceptual and behavioral adaptation to inversion of incoming light.

In a related study, Brown (1928) wore goggles with prisms that rotated incoming light $75^{\circ}$ around the line of sight for one week, and he adapted to this rotation to a significant degree. This adaptation occurred even though he described his apparatus as "too unwieldy" (p. 134) to wear every night on a one-half mile trip to his university where various tests were run. Other work investigating adaptation of the visual system to alterations in incoming light that did not involve inversion of this light has also indicated a very high degree of flexibility in the operation of the visual system (e.g., Gibson, 1933; Held, 1965; Held \& Freedman, 1963).

Stratton (1899) conducted an experiment in which through the use of a specific arrangement of mirrors his body image and immediate surroundings were projected in what without adaptation would be the horizontal direction out from his body at the level just above his shoulders, his head in the reflected image of his body closest to his body and the front of his body facing up. In the very brief time that Stratton wore the mirror arrangement, he found that visual perception and visually guided action showed significant adaptation to the incoming light. Stratton wrote:

The whole experience [in this experiment] was thus so similar to the one with the inverting lenses that I hesitate to present it even in this brief outline. But under the circumstances the very similarity is a distinct addition to the data from the previous experiment, since it shows that the introduction of the new factor--that of distance--does not prevent an ultimate spatial concord [between the haptic, kinesthetic, and visual senses]....The experiment thus suggests that the principle stated in an earlier paper--that in the end we would feel a thing to be wherever we constantly saw it--can be justified in a wider sense that I then intended it to be taken. (Stratton, 1899, pp. 497-498) 


\section{Can Psychology}

Ewert (1930) and Munn (1955/1965) have disputed the finding that visual experience becomes upright after experience with rotated incoming light. It should be noted, though, that the major concern of Ewert and Munn is not so much the subject's phenomenal experience with rotated light but rather with the interpretation of what this phenomenal experience means. For example, Munn (1955/1965), who was one of Ewert's subjects, wrote:

Localizing reactions became so automatic at times that a "feeling of normalcy" was present. This is probably the feeling reported by Stratton and interpreted as "seeing right-side up." (p. 293)

Or, Ewert concluded that:

In all forms of activity where overt localizing responses are present there is rapid adjustment to the distracting visual interference until at the end of 14 days of practice the interference is entirely overcome in some of the activities investigated and almost overcome in the other forms....Constant interference during visual disorientation does not prevent the steady growth of a habit. (Ewert, 1930, pp. 353; 357)

Snyder and Pronko (1952) performed an experiment similar in many respects to Ewert's. Munn (1955/1965) wrote about Snyder and Pronko's work: "The results were essentially like his [Ewert's]" (p. 294). In contrast to Ewert, Snyder and Pronko concluded:

It appears that perceivings form a behavior sequence going back into the individual's past. If the subject of the present experiment had always worn the inverting lenses, his past perceivings would have been of a piece with those of the moment when the question ["Well, how do things look to you? Are they upside-down?" (p. 113)] was directed at him. Obviously, then, they would not have been in contrast with the latter and would not have called attention to themselves. Stated in another way, if this subject had somehow developed amnesia at the point at which he put on the inverting lenses, then things could not appear upside-down because there would be no basis of comparison or contrast. That they did appear upside-down is clearly a strict function of his previously acquired perceivings. (pp. 113-114) 


\section{Chapter 7}

In A History of Experimental Psychology, Boring (1929/1950) wrote about Stratton's work:

In 1896 Stratton put the matter to test, having his subjects [actually only Stratton himself] wear a system of lenses which reversed the retinal image and made it right side up. The expected happened. The perceived world looked upside down for a time and then became reversed. Taking the glasses off resulted once again in reversal which was soon corrected. Stratton was not, however, confused by the homunculus. He described how up was nothing in the visual sensory pattern other than the opposite of down, and that orientation is achieved by the relation of the visual pattern to somothesis and behavior. When you reach up to get an object imaged at the top of the retina, then you have indeed got the visual field reversed and will not find the object unless you have on Stratton's lenses. Ewert repeated this experiment in 1930, with similar results [italics added]....Had the view of a freely perceiving agent in the brain not been so strongly entrenched, this problem could not have continued to seem so important in 1604, 1691, 1709, 1838, 1896 and 1930. (p. 678)

Boring knew of Ewert's work and saw that the empirical results obtained by Ewert supported Stratton's conclusion even if Ewert's own conclusion based on the empirical results he found was not in agreement with Stratton's. Dolezal (1982) wrote concerning the results of his experiment and those found in other experiments involving the inversion of incoming light to the observer:

In the course of living in a world transformed, the observer's initial fears become calmed, he or she finds the discomforts quite tolerable, the strange sights fade and become common, and ineptness changes to competency. (p. 301)

\section{A Biperceptual Capability}

Dolezal proposed that the observer who adapts to inversion of incoming light is biperceptual and biperformatory. Biperceptual refers to the simultaneous existence of the visual perceptual capabilities associated with both pre-inversion and inversion conditions. These capabilities may be considered distinct reference frames for the individual who has undergone inversion of 


\section{Can Psychology}

incoming light. Similarly, biperformatory refers to the simultaneous existence of an individual's capabilities to act competently in the environment both before and after inversion of the incoming light. These capabilities as well are divided into distinct frameworks for the individual who has experienced and adapted to this inversion of incoming light. Dolezal (1982) wrote:

The adapted observer appears to differ from the unadapted observer in several main respects. After some 200 hours of living with reversing prisms, an observer once again experiences visual stability of the perturbed environment [i.e., up-down reversal of incoming light]. This is true for a wide range of rates of head movements (HMs). Moreover, the adapted observer has acquired what may be called another "personality" (i.e., he or she has the dual facility to be perceptually and emotionally comfortable and to act competently both with and without transforming prisms). The adapted observer is thus a very different creature from the unadapted observer--somewhat like someone with a second language or a novel set of skills that can only be directly displayed under special circumstances (cf. statedependent learning and recall). The observer becomes what I call biperceptual and biperformatory....In general, the adapted observer is capable of living in both worlds, under both sets of information conditions and behavioral requirements with roughly equal comfort and competence. (p. 297)

Consider the following observation reported by the subject in Snyder and Pronko's study, who happened to be Snyder, that supports Dolezal's thesis:

Toward the end of the experiment [i.e., the period in which the subject wore the inverting glasses], the subject was adequately adjusted [adapted]. The following insightful experience occurred. He was observing the scene from a tall building. Suddenly someone asked, "Well, how do things look to you? Are they upside-down?"

The subject replied, “I wish you hadn't asked me. Things were all right until you popped the question at me. Now, when I recall how they did look before I put on these lenses, I must answer that they do look upside down now. But until the 


\section{Chapter 7}

moment that you asked me I was absolutely unaware of it and hadn't given a thought to the question of whether things were right-side-up or upside-down." (Snyder \& Pronko, 1952, p. 113)

In a study of retention of the effects of such inversion, Snyder and Snyder (1957) found that when the inverted conditions were reintroduced for a subject some time after the subject's initial experience with inverted light, the subject's adjustment the second time to the inverted light indicated that learning occurred as a result of the first experience and had been retained over a two-year period between the first and second experiences with inversion of the incoming light. Specifically, Snyder and Snyder found that the time to complete various tasks consistently took less time in the second experience than in the first. The learning curves in the first and second experiences were very similar for each of the tasks, only in the second exposure the times to complete the tasks were consistently lower than the times to complete them in the first exposure.

In his research, Stratton noted how quickly the perceptual framework of the subject exposed to inverted incoming light could switch between the unadapted and the adapted orientation. He also noted the possibility of their coexistence. For example, on the seventh day of wearing his optical apparatus in his second experiment with inverted light, Stratton (1897b) wrote:

When I watched one of my limbs in motion, no involuntary suggestion arose that it was in any other place or moved in any other direction than as sight actually reported it, except that in moving my arm a slightly discordant group of sensations came from my unseen shoulder. If, while looking at the member, I summoned an image of it in its old position, then I could feel the limb there too. But this latter was a relatively weak affair, and cost effort. When I looked away from it, however, I involuntarily felt it in its pre-experimental position, although at the same time conscious of a solicitation to feel it in its new position. This representation of the moving part in terms of the new vision waxed and waned in strength, so that it was sometimes more vivid than the old, and sometimes even completely overshadowed it. (p. 465)

It is remarkable that the visual system has demonstrated a great degree of flexibility in the inversion experiments given the degree of artificiality 


\section{Can Psychology}

introduced into the experimental circumstances by the optical apparatuses that have been used. For example, in the inverted light experiments, Stratton used a device that allowed for incoming light to only one eye while the other eye was covered. Ewert's device was lightweight but allowed for a limited visual field. In an attempt to widen the visual field over that of most other experiments in which all incoming light is inverted for an extended period of time, Dolezal (1982) built his optical device out of a football helmet and inserted glass prisms in the limited space usually left open for a football player to see. His device weighed 8 pounds, 6 ounces. There is further work to be done concerning the effect of inverted light on visual experience and visually guided action. But the basic result that there is significant adaptation in visual experience and visually guided action to inversion of incoming light has been established (Snyder, 1992, 1993, 1995).

\section{Hard Wiring of the Visual System \\ and the Isotropy of Space}

Originally, Stratton was concerned with showing that two theories concerning inversion of incoming light were incorrect. Essentially, these theories maintained some sort of hard-wiring of either the neural component of the visual system (the projection theory) or its supporting musculature (the eye movement theory). In the projection theory, inversion of the retinal image was needed because of the crossing of the lines of direction of light from external objects when light from the external world moves through the eye. Perception was considered to depend on these lines of direction that projected outward to the upright objects in the physical world from which the light rays originated.

The eye movement theory related to the use of the musculature about the eye to provide definitive information about the correct position of objects in the world. Thus, if the eyes move upward in their sockets, they see the upper parts of objects in the physical world, and if the eyes move downward in their sockets, they see the lower part of these objects in the physical world. In this process, though, movement of the eye upward, for example, results in the lower portion of the retina receiving more of the incoming light. Inversion of the incoming light would help to correct this problem and could provide the basis for indicating the upright nature of the physical world.

Many scholars have believed the tenet behind the projection and eye movement theories that there is only one way the visual system can function in order to perceive the physical world as upright. This is an assumption that 


\section{Chapter 7}

Boring (1929/1950) maintained was based on the notion of the homunculus. It can be seen in the descriptions of the projection and eye movement theories that they both have another more basic tenet as an assumption regarding the physical world. This assumption is that the physical world itself has an absolute status regarding its being upright. For example, if the physical world were indeed upside down, would scholars seriously entertain a theory of visual perception based on the hard wiring of the visual system? The assumption that the physical world has an absolute status regarding its being upright violates the isotropy of space in that space is not fundamentally the same in different directions.

\section{Manipulating Visual Perception in the Investigation of a Physical System}

How does this adaptability of visual experience and visually guided behavior to inversion of incoming light help in the analysis of modern physical theory? Consider the spin angular momentum of an electron, a quantity intrinsic to the electron. (A classical analog to this quantity would be the angular momentum of a spinning top.) Quantum mechanics predicts that it is possible to measure the component of this momentum along any one of three orthogonal axes, $x, y$, and $z$ (three spatial axes all at right angles to one another). The spin component of the electron along the $z$ axis, or any other spatial axis, can have one of two values when measured (i.e., $+1 / 2 h / 2 \pi$ and $-1 / 2 h / 2 \pi)$. Assume in an experiment that this measurement of the electron occurs in the following way. Through the use of a Stern-Gerlach apparatus (Eisberg and Resnick, 1974/1985; Liboff, 1993), a nonuniform magnetic field oriented along the $z$ axis is placed in the path of the electron. The value of the spin component of an electron along the $z$ axis can be determined with the Stern-Gerlach apparatus because the electron will take one path in the apparatus if it has one value and will take another path in the apparatus if it has the other value. As an electron exits the apparatus from one of two locations, indicating which one of two paths the electron took through the apparatus and thus the value of its spin component along the axis of the magnetic field of the apparatus, detectors placed at the exits of the apparatus detect the electron. A human observer inspecting these detectors at the exits of the Stern-Gerlach apparatus then can determine which path the electron took and the value of its spin component along the axis of the magnetic field of the apparatus and its gradient. 


\section{Can Psychology}

Assume the $z$ axis is in the vertical direction relative to the subject, appearing to go up and down. The two values of the spin component along the $z$ axis can be designated spin up or spin down according to the motion of electrons passing through the Stern-Gerlach apparatus relative to the vertical axis. Assume that the $y$ axis runs perpendicular to the ideal plane formed by the subject's face and that prior to entering the nonuniform magnetic field the electron is traveling along this axis. Assume that the $x$ axis runs horizontally relative to the subject, from side to side. The experimental circumstances are depicted in Figure 31, where + and - refer to the positive and negative directions along a spatial axis. According to quantum mechanics, precise knowledge resulting from measurement of one of the spin components means that knowledge of each of the other two spin components is completely uncertain. Precise knowledge of the component along the $z$ axis, for example, means that knowledge of each of the components along the $x$ and $y$ axes is completely uncertain.

This limitation concerning the knowledge of certain paired quantities in quantum mechanics also characterizes the simultaneous precise determination of the position and momentum of an electron along a spatial axis (Eisberg and Resnick, 1985; Liboff, 1992). Precise knowledge of the electron's momentum entails complete uncertainty regarding its position, and precise knowledge of its position entails complete uncertainty regarding its momentum. As Einstein, Podolsky, and Rosen (1935) wrote:

It is shown in quantum mechanics that, if the operators corresponding to two physical quantities, say $A$ and $B$, do not commute, that is, if $A B \neq B A$, then the precise knowledge of one of them precludes such a knowledge of the other. Furthermore, any attempt to determine the latter experimentally will alter the state of the system in such a way as to destroy the knowledge of the first. (p. 778)

Where $\mathrm{A}$ and $\mathrm{B}$ are the operators corresponding to the components of the position and momentum of an electron, respectively, along a particular spatial axis, they do not commute. Similarly, where A and B are the operators for any two components along orthogonal spatial axes of the spin angular momentum of an electron, they do not commute.

One can use an apparatus like that developed by Stratton to rotate the incoming light to an experimental subject such that the light is rotated around the 


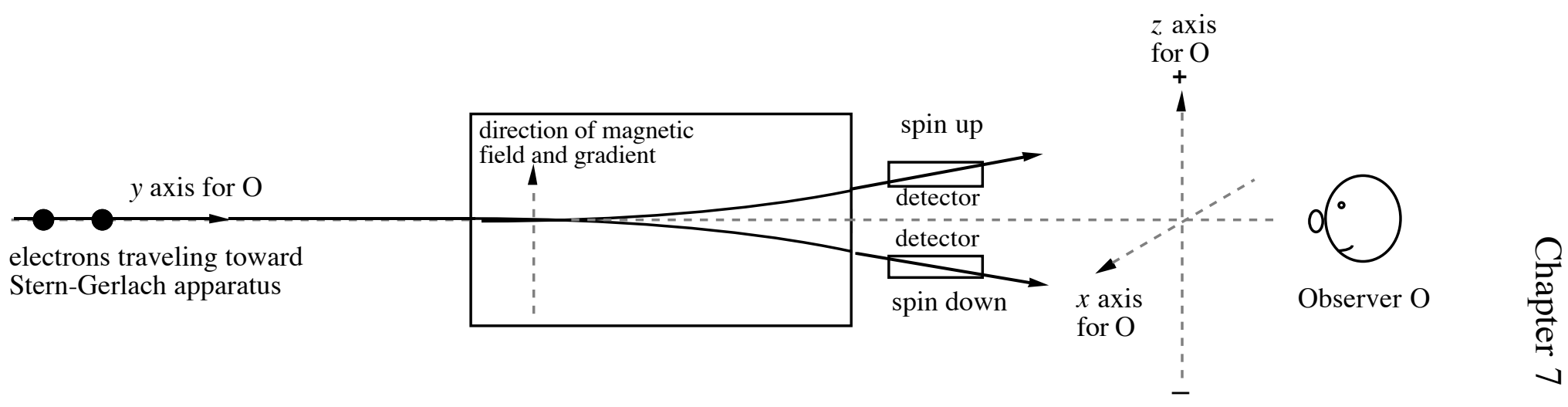

Figure 31

Observer $\mathrm{O}$, not wearing the optical apparatus, viewing the spin component along the $z$ axis in the spatial structure of $\mathrm{O}$ of electrons traveling through a Stern-Gerlach apparatus without rotation of the visual field. 


\section{Can Psychology}

$y$ axis $90^{\circ}$. Then, what was information concerning the $z$ axis is now information concerning the $x$ axis as concerns the light impinging on the subject's retina. Given Stratton's results, there is a good possibility that, after a period of orientation with this apparatus, particularly if it is worn for an uninterrupted period and the subject is allowed to move freely in the natural environment, the subject will see the electron moving up or down and not sideways.

Indeed, the natural scenarios tested by Stratton and others are much more complex than the scenario need be that could be presented to an observer in a laboratory setting observing the path of an electron along a spatial axis in an inhomogeneous magnetic field like that created by a Stern-Gerlach apparatus. It does appear that adaptation to inverted light depends to a significant degree on a subject's experience in moving in his or her natural environment while incoming light is inverted by the optical apparatus. Because of the uncomplicated nature of the adaptation needed in the proposed experiment, though, the amount of sensorimotor experience in the natural environment required by the subject for the necessary degree of adaptation of the visual system to occur should not be great. It should be emphasized that research has indicated that the shift in the spatial structure of the observer that depends on sensorimotor experience involves variables pertaining to the individual, for example the correspondence of kinesthetic and haptic sensation with visual perception that develops over time. This correspondence among sensory modalities is not determined by the object or objects in the physical world that are perceived.

Once there is a significant degree of adaptation in the subject's visual experience, according to the information impinging on the subject's retina, the subject is measuring what in the original situation without rotation of the incoming light is the $x$ axis (Figure 32). What for observers in the original situation is up and down along the $z$ axis is for the subject up and down along the $x$ axis, where the $x$ axis is labeled in terms of the spatial structure associated with the observer who does not wear the optical apparatus. For the subject wearing the optical apparatus and adapted to the rotated incoming light, the spin components along the $y$ and $z$ axes of the spatial structure associated with the observer who does not wear the optical apparatus are completely uncertain.

It is important to note that for the observer wearing the optical apparatus and adapted to the rotated light, in his or her shifted spatial structure, the $z$ axis runs along the vertical or upright direction just as the $z$ axis runs along the 


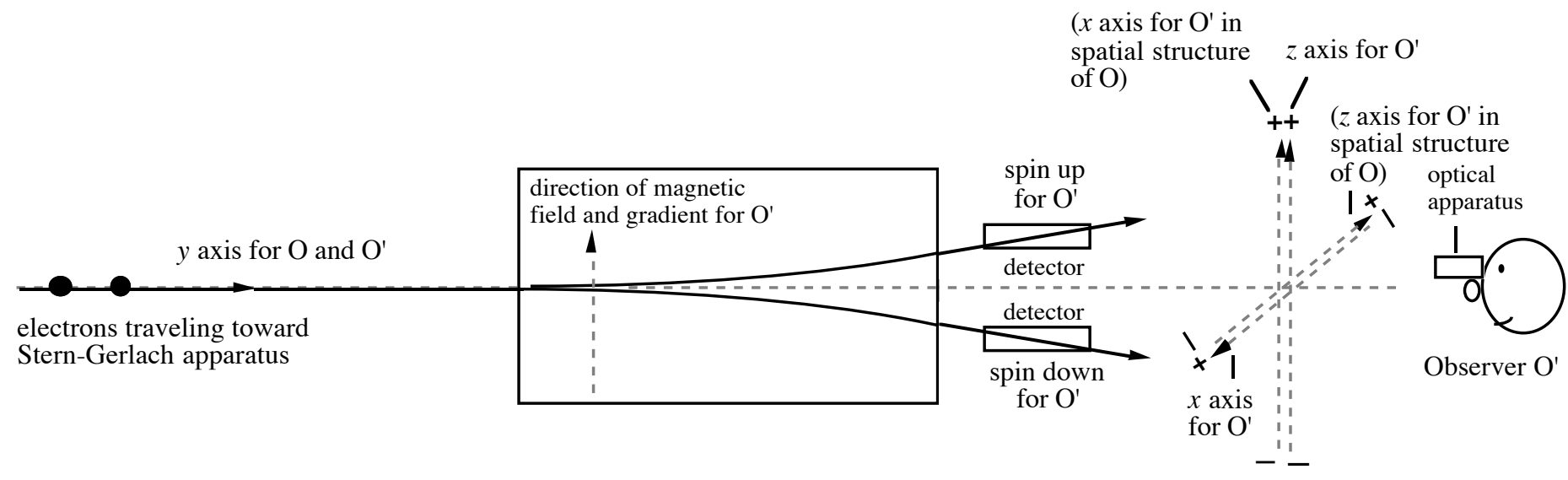

Figure 32

Observer $\mathrm{O}^{\prime}$, adapted to wearing the optical apparatus, viewing the spin component along the $z$ axis in the spatial structure of $\mathrm{O}^{\prime}$ of electrons traveling through a Stern-Gerlach apparatus with 90 degree rotation of the visual field, in terms of the spatial structure of $\mathrm{O}$, around the $y$ axis. $(x$ and $z$ axes for $\mathrm{O}^{\prime}$ in spatial structure of $\mathrm{O}$, who does not wear the optical apparatus, are shown.) 


\section{Can Psychology}

vertical or upright direction for the observer who does not wear the optical apparatus. The observer adapted to the incoming light and wearing the optical apparatus measures the spin component in his or her shifted spatial structure along the $z$ axis, just as the observer who does not wear the optical apparatus measures the spin component along the $z$ axis in his or her spatial structure.

It appears possible to know the precise values for two components along orthogonal axes of the spin angular momentum of an electron at the same time for a particular spatial structure, where one takes into account one of the orthogonal axes as it is perceived by an observer using a different spatial structure. The key to this gedankenexperiment is that a basic shift has occurred in the spatial structure for the observer who wears the optical apparatus and adapts to the rotated incoming light. This shifted spatial structure has the same significance for this observer as the spatial structure of the observer who does not wear the optical apparatus has for this latter observer. This basic gedankenexperiment shows in a particular instance that it is possible that the uncertainty principle is conditioned by a link between the spatial structure of an observer in the physical world and the orientation of incoming light relative to the observer. Of particular importance is the evidence that there are factors specific to the observer in the development of an individual's spatial structure.

Evidence supporting a biperceptual character of visual perception after adaptation to inversion of incoming light has been noted. This biperceptual character of visual perception concerns the simultaneous existence of the distinct visual perceptual capabilities associated with both pre-inversion and postinversion conditions. It may be possible for one subject in the experiment outlined above involving spin angular momentum components along orthogonal spatial axes to be involved in mutually exclusive situations simultaneously concerning the same concrete physical circumstances. That is, the adapted subject may be able to instantly shift from being involved in one of the experimental scenarios to the other.

\section{A $180^{\circ}$ Rotation}

There is another circumstance besides that already discussed that is perhaps even more surprising. Consider that an optical apparatus is used that rotates incoming light $180^{\circ}$ around the line of sight (Figure 33). For the subject wearing the apparatus but not yet adapted, the negative direction of the $z$ axis is associated with spin up and the positive direction of the $z$ axis is associated with spin down. Once a significant degree of adaptation in the subject's visual 


\section{Chapter 7}

experience occurs, when the subject observes that an electron has spin up in the positive direction of the $z$ axis, according to the information impinging on the subject's retina the subject is measuring what in the original situation without rotation of the incoming light is spin up in the negative direction of the $z$ axis. Similarly, when the subject observes that an electron has spin down in the negative direction of the $z$ axis, according to the information impinging on the subject's retina the subject is measuring what in the original situation without rotation of the incoming light is spin down in the positive direction of the $z$ axis.

If this result is considered in terms of the Schrödinger cat gedankenexperiment (Schrödinger, 1935/1983), it is as if in one situation, one atom of the radioactive material decayed leading to the cat's being dead when observed. Simultaneously, in the other situation, none of the radioactive material decayed, leading to the cat being alive when observed. One situation involves the observer who has not worn the optical device and who is not wearing the device when he or she observes the electron. The other situation involves the observer who is wearing the apparatus when observing the electron and who has adapted to the rotated visual field. In contrast to the Schrödinger cat gedankenexperiment, each observer's perception is similar in structure. But while the effect of the rotation of incoming light for a subject is mitigated upon adaptation, the historical physical event of the rotation of the incoming light due to the optical apparatus remains for the observer wearing the device and acts to distinguish the two situations of the observers.

Practically speaking, the "righting" of the visual field for the adapted subject means that the electron with spin up in the original spatial structure now has spin down in the original spatial structure even though the electron has spin up in the spatial structure of the adapted observer. For the subject wearing the optical apparatus but who has not adapted to the inversion of incoming light, the spatial structure of the world is the same as that for the subject who does not wear the optical apparatus and for whom light is not inverted. The unadapted subject knows that his visual experience is altered by wearing the optical apparatus but that the world itself remains unchanged. The unadapted subject knows that the spin component of the electron is up, that is up for an observer not wearing the optical apparatus, though it appears to be down to him, opposite to the direction found by an observer not wearing the optical apparatus.

But with adaptation to inverted incoming light, including the sense of normalcy in visual experience and competency in visually guided action that 


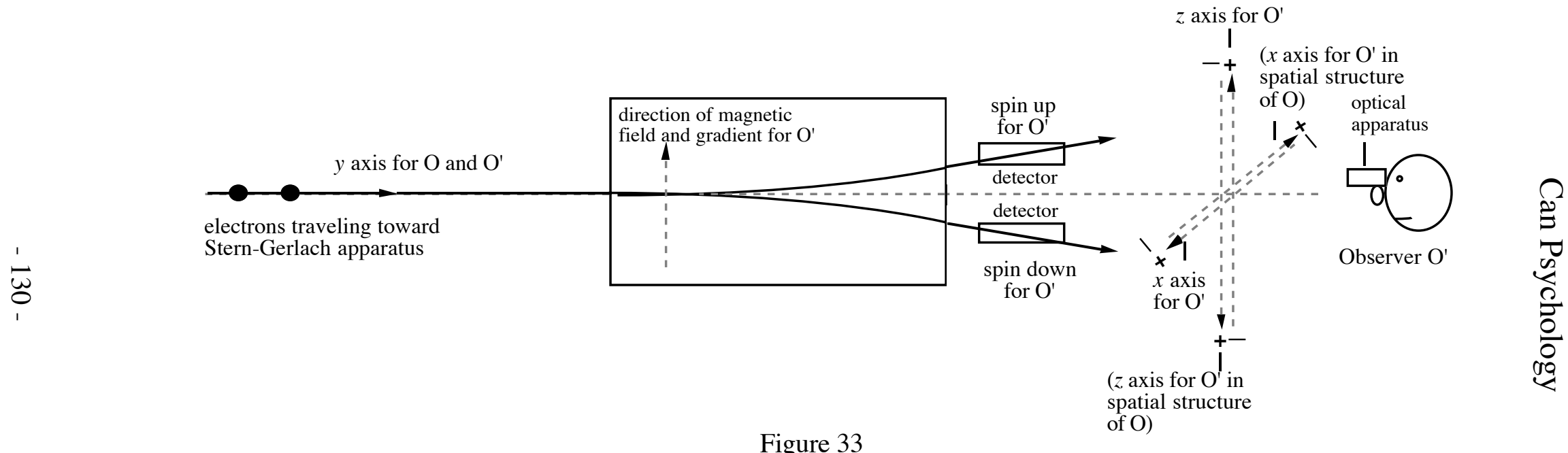

Observer $\mathrm{O}^{\prime}$, adapted to wearing the optical apparatus, viewing the spin component along the $z$ axis in the spatial structure of $\mathrm{O}^{\prime}$ of electrons traveling through a Stern-Gerlach apparatus with 180 degree rotation of incoming light, in terms of the spatial structure of $\mathrm{O}$, around the $y$ axis. ( $x$ and $z$ axes for $\mathrm{O}^{\prime}$ in spatial structure of $\mathrm{O}$, who does not wear the optical apparatus, are shown.) 


\section{Chapter 7}

supports this sense of normalcy, what appears as a spin up component for the electron to the adapted observer is a spin down component when considered in terms of the original spatial structure, the spatial structure of the subject for whom incoming light is not inverted. Unlike the unadapted observer, the adapted observer does not maintain that the world is unchanged while his visual experience has been altered by inverting incoming light with the use of the optical apparatus. The adapted observer experiences the "real" direction of the spin component opposite to that experienced by the unadapted observer. In terms of the impact of incoming light on his retina, the adapted observer who observes a spin up component experiences a spin component in the opposite direction to that which the same pattern of light on the retina supports for the unadapted observer (Snyder, 1995).

\section{A Possible Objection}

One might object to this conclusion regarding the "righting" of the visual field for the adapted subject in the following way. The artificial reorientation of the incoming light does not prevent tracing back light impacting the retina to the physical existent that is the source of the light so that the actual spin component of the electron along a particular axis in space and the visual perceptions of the electron's spin component in space by the observer for whom light is not inverted, or otherwise rotated, and by the individual adapted to the incoming, altered light are all in agreement. That is, both observers correctly deduce the spin component of the electron because they correctly perceive the motion of the electron in space along the spatial axis along which it is traveling. Then the visual system could be said to simply adapt to the artificial change in incoming light but that the internal sensory coordination by the observer ultimately reflects the absolute positioning of the electron's motion in space as it moves through the Stern-Gerlach device.

But, consider that instead of using an external optical system to alter the orientation of incoming light, another method is used in the gedankenexperiment to change the orientation of light on the retina. Consider that the retinas of an observer with their supporting physiological structures in the eye, in particular the optic nerve, are rotated $180^{\circ}$. In this arrangement, an optical apparatus external to the visual system is no longer used. (Both procedures accomplish the same goal, rotation of incoming light $180^{\circ}$ on the retina.) The results, though, of using either the external optical system or reorienting the retinas themselves should be the same. The human subject whose retinas have been reoriented should with adaptation see the world upright even though the 


\section{Can Psychology}

incoming light falls on the retinas in the opposite manner to that found for the observer whose retinas have not been reoriented, where space is considered in terms of the spatial structure of this latter observer. By reorienting the retinas themselves, one can no longer reasonably subscribe to the thesis that the visual system is simply accounting for the artificial nature of the external optical apparatus in order to correctly ascertain the absolute positioning of the electron's motion in space. Here, no extra instrument is added. Only the orientation of the incoming light relative to the retina has been changed.

\section{Summary of the Results of the Gedankenexperiments}

Psychology can add in a unique way to the exploration of the link between cognition and the physical world found in modern physical theory. Developing an example of an experiment that combines methodology from both psychology and physics has provided an interesting result. The gedankenexperiments presented have indicated the importance of an individual's spatial structure in measurement in quantum mechanics. Two fundamental features of quantum mechanics are:

1) quantities represented by non-commuting Hermitian operators cannot be simultaneously known;

2) only one value from a set of mutually exclusive values for a quantity can be known at a time.

The gedankenexperiments indicate that both of these features are conditioned by a link between the spatial structure of an observer in the physical world and the orientation of incoming light relative to the observer. An individual's spatial structure itself is dependent on variables pertaining to the observer, for example, the correspondence of the haptic, kinesthetic, and visual senses. Within a particular spatial structure, the two fundamental features of quantum mechanics are correct. Yet, when different spatial structures of different observers, or different spatial structures of the same individual who is adapted to wearing the optical apparatus, are compared, it is likely that these features can be modified. Instead, in these latter circumstances, it is possible that:

1) quantities represented by non-commuting Hermitian operators can be simultaneously known when these quantities are considered in different spatial structures; 


\section{Chapter 7}

2) more than one value for a quantity from a set of what would be in one spatial structure mutually exclusive values can be known at a time.

The model for developing the gedankenexperiments presented is based on adjusting the observer variables affecting the act of perception. The data obtained in the measurement process concern quantities of the measured physical system, and the expected empirical results are those predicted in quantum mechanics. This model contrasts with most research in quantum mechanics in which the focus is on adjusting variables directly affecting the physical system measured or a physical apparatus directly acting as a measuring instrument. 


\section{Chapter 8}

\section{Conclusion}

There is more. There is, for example, the implication for the nature of time implied by the fundamentally probabilistic character of statistical mechanics and the reliance of physicists on the second law of thermodynamics as the basis for the directionality of time. Given these characteristics, can the objective character that has been granted to the directionality of time be considered valid? Physicists have relied on this objective character in maintaining that time has an absolute direction. As has been discussed, an absolute direction is not found in physical law. Except in one area that is not directly relevant to everyday life, physical law itself is reversible in time. Moreover, research has indicated that in certain circumstances the increasing disorder characterizing a physical system can be reversed if an outside influence is introduced to the system (Snyder, 1987).

Where does the exploration of physical theory that we have engaged in leave us? It leaves us with the reasonably drawn conclusion that the mind is fundamentally linked to the physical world. A cognitive link to the physical world in physical theory eliminates the artificial separation between the object as perceived and its "real" existence in a world essentially independent of the perceiving individual and which somehow supports the perception. This thesis is not solipsistic because physical theory provides avenues for empirical verification. The physical world answers empirical queries put to it on its own.

Considered in the context that a link between the mind and the physical world is quite natural, the fact that empirical test has provided a great deal of support for modern physical theory is not surprising. This link provides the conceptual basis to resolve what has been a significant difficulty concerning the relation of the physical world to the perceiving individual that has characterized physical theory at least since the development of Newtonian mechanics.

A recurring theme in our exploration of physical theory has been a certain flexibility that an individual has in his interaction with the physical world. There is, of course, the flexibility in the structure of the reference frame used by an individual. This flexibility is associated with individuals at rest in their inertial reference frames in uniform translational motion relative to one another believing that their respective reference frames are "stationary" while the other observers' reference frames are "moving." This flexibility in the reference frame and temporal coordinate system allows for the relativity of simultaneity in 


\section{Conclusion}

the special theory to be argued with either of the two inertial reference frames in uniform translational motion relative to one another to be the "stationary" reference frame. It is flexibility in the structure of the reference frame used by an individual that allows for the equivalence of inertial and non-inertial reference frames in the description of physical phenomena through the particular equivalence of a uniformly accelerating reference frame and an inertial reference frame experiencing a uniform gravitational field. In quantum mechanics, the choice of what quantity to measure has significant consequences for the physical world. Bohr referred to this choice as a "free choice" (Bohr, 1935, p. 698). In statistical mechanics, there is a fundamental difference in considering a macroscopic system in its entirety or bit by bit in terms of its microscopic elements.

Man has accomplished a great deal by considering the mind fundamentally separated from the physical world. There is much that he can accomplish by considering his cognition fundamentally related to the physical world. Puzzlement regarding how various empirical results could occur for those who continue to hold that the physical world is independent of mind will be replaced by the systematic investigation of the particular relationships linking cognition and the physical world.

Physicists in the nineteenth century, such as Helmholtz and Fechner, made fundamental contributions to the founding of psychology as an independent discipline (Boring, 1929/1950). In contrast to Helmholtz and Fechner, physicists since the early part of the 20th century have had, for the most part, little expertise concerning psychological phenomena. They have not had the sensitivity to these phenomena that results from years of formal training, and they do not know how to investigate these phenomena systematically. Moreover, the lack of knowledge by most psychologists concerning modern physical theory, and much of the mathematics in which it is expressed, has prevented psychologists in large part from providing initial guideposts on how a psychological factor in the structure and functioning of the physical world might be explored.

Yet, early on, major figures in the development of quantum mechanics (e.g., Bohr, Schrödinger, Einstein, and Heisenberg) realized that the measurement process was central to the concept of physical reality in quantum mechanics and recognized that a link between cognition and the physical world was not an unnatural possibility in quantum mechanics, even if they maintained that it was not in fact the case. For example, in describing 


\section{Chapter 8}

gedankenexperiments like those described in Gedankenexperiments 1 and 2 in Chapter 5, "Quantum Mechanics," Bohr wrote:

It is only the circumstance that we are presented with a choice of either tracing the path of a particle or observing interference effects, which allows us to escape from the paradoxical necessity of concluding that the behaviour of an electron or a photon should depend on the presence of a slit in the diaphragm through which it could not be proved to pass [italics added]. (Bohr, 1949/1969, pp. 217-218)

This realization does not seem to have eluded contemporary physicists as well. For example, Mermin (1985) quoted Greenberger, a physicist who said, "Quantum mechanics is magic" (p. 38). Or Feynman (1982) wrote:

We always have had (secret, secret, close the doors!) we always have had a great deal of difficulty understanding the world view that quantum mechanics represents. At least I do, because I'm an old enough man that I haven't got to the point that this stuff is obvious to me. Okay, I still get nervous with it. And therefore, some of the younger students...you know how it always is, every new idea, it takes a generation or two until it becomes obvious that there's no real [italics added] problem. It has not yet become obvious to me that there's no real [italics added] problem. I cannot define the real [italics added] problem, therefore I suspect there's no real [italics added] problem, but I'm note [sic] sure there's no real [italics added] problem. (p. 471)

That Feynman (1982) cannot define the "real problem" (p. 471) and is "not sure there's no real problem" (p. 471) is the case in part because physicists have maintained a stance that does not allow for a straightforward explanation of the quantum mechanical wave function. Feynman's repeated use of the word "real" signifies his acknowledgment that the primary issue of concern to him is whether the physical world in quantum mechanics exists independently of the individual who is thinking about it and observing it.

Psychology has a unique role to play in understanding the relationship between cognition and the physical world in modern physical theory. The work of Stratton and others on the inversion of incoming light, and the work of Cooper and her colleagues on mental rotation, are examples of this unique role. 


\section{Conclusion}

Psychologists have the training and experience to focus on the cognitive acts through which knowledge of the physical world is gained. Being familiar with the body of psychological knowledge, they can quickly access that knowledge that may well be relevant, and they are familiar with the research methodologies that can be used to investigate possible connections. This endeavor, though, requires psychologists to learn the fundamentals of modern physical theory and to become knowledgeable with the basic mathematical tools central to the description of this theory. The collaboration of physicists and psychologists will be necessary to develop a thorough understanding of the irreducible connection between cognition and the physical world indicated in modern physical theory.

The isolation of man from the physical world that has been a central factor of experience will be supplanted by an acknowledgment of the fundamental link between mind and the physical world. At the same time, new questions arise. For example, what is it that I as an observer can actually affect in the constitution of the world? In my relationship to the world, what is the nature of the boundary between the world and myself? What is my relationship to other people whose mental processes also are fundamentally linked to the physical world? These questions will require systematic inquiry by physicists and psychologists. However one feels about the new questions that must be asked, one can no longer reasonably refrain from asking the questions. The

evidence from modern physics is clear: the mind of man is directly linked to the structure and functioning of the physical world. 


\section{Appendix A}

\section{The Association of Length and Duration with the Logical Roles of Inertial Reference Frames in the Argument on the Relativity of Simultaneity}

\begin{tabular}{|c|c|c|}
\hline \multicolumn{3}{|c|}{$\begin{array}{c}\text { TABLE 1: } \\
\text { SCENARIO } 1\end{array}$} \\
\hline $\begin{array}{l}\text { Inertial Reference } \\
\text { Frame: }\end{array}$ & W & $\mathrm{W}^{\prime}$ \\
\hline $\begin{array}{l}\text { Logical Role in } \\
\text { Derivation of } \\
\text { Relativity of } \\
\text { Simultaneity: }\end{array}$ & $\begin{array}{l}\text { "Stationary" frame. } \\
\text { Simultaneity established first in } \\
\text { this frame. }\end{array}$ & $\begin{array}{l}\text { "Moving" frame. } \\
\text { Time in W used to determine } \\
\text { whether criterion for simultaneity } \\
\text { met in W' (using same light used to } \\
\text { establish simultaneity in W). }\end{array}$ \\
\hline $\begin{array}{l}\text { State of Motion of } \\
\text { Rod Measured in } \\
\text { Equation 4: }\end{array}$ & $\begin{array}{l}\text { Rod measured by observer at rest } \\
\text { in } \mathrm{W} \text { is "moving" relative to } \mathrm{W} . \\
\Delta x \text { is determined for "moving" } \\
\text { measuring rod. Need to determine } \\
\text { positions of ends of rod in W } \\
\text { simultaneously. } \\
\text { To measure } \Delta x \text {, observer at rest in } \\
\mathrm{W} \text { uses rod of identical } \\
\text { construction to the rod being } \\
\text { measured. This rod used to } \\
\text { measure } \Delta x \text { is at rest in W. } \\
\text { The length of the rod "moving" in } \\
\mathrm{W} \text { is shorter when measured by an } \\
\text { observer at rest in W than when } \\
\text { measured by an observer at rest in } \\
\text { W'. }\end{array}$ & $\begin{array}{l}\text { Rod measured by observer at rest in } \\
\text { W is at rest in W'. } \\
\text { The length of this rod at rest in } W^{\prime} \\
\text { is longer when measured by an } \\
\text { observer at rest in } W^{\prime} \text { than when } \\
\text { measured by an observer at rest in } \\
\text { W. } \\
\text { This rod that is measured and that is } \\
\text { of identical construction to the rod } \\
\text { at rest in W that is used to measure } \\
\text { the former rod in W is in Scenario } \\
2 \text {, instead, used to measure the } \\
\text { length of the latter rod (the rod at } \\
\text { rest in W) where the latter rod is the } \\
\text { "moving" rod. }\end{array}$ \\
\hline
\end{tabular}




\section{Appendix A}

\begin{tabular}{|c|c|c|}
\hline \multicolumn{3}{|c|}{$\begin{array}{l}\text { TABLE } 1 \text { (cont'd) } \\
\text { SCENARIO 1: }\end{array}$} \\
\hline $\begin{array}{l}\text { Inertial Reference } \\
\text { Frame: }\end{array}$ & $\mathrm{W}$ & $\mathrm{W}^{\prime}$ \\
\hline $\begin{array}{l}\text { State of Motion of } \\
\text { Clocks Measured } \\
\text { in Equation 2: }\end{array}$ & $\begin{array}{l}\text { Clock (or clocks) measured by } \\
\text { observer at rest in W' is "moving" } \\
\text { relative to W. } \\
\text { Clocks at rest in W are used to } \\
\text { determine } \Delta t \text { corresponding to } \Delta t^{\prime} \\
\text { in } \mathrm{W}^{\prime} \text {. Observers at rest in W' use } \\
\text { clocks of identical construction to } \\
\text { that (or those) clock (or clocks) at } \\
\text { rest in W' that is (or are) being } \\
\text { measured. } \\
\text { The rate of the clock (or clocks) } \\
\text { "moving" in W is (or are) slower } \\
\text { when measured by an observer at } \\
\text { rest in W than when measured by } \\
\text { an observer at rest in W'. }\end{array}$ & $\begin{array}{l}\text { Clock (or clocks) measured by } \\
\text { observer at rest in W is at rest in } \\
\text { W'. } \\
\text { The rate of this clock (or clocks) at } \\
\text { rest in W' is faster when measured } \\
\text { by an observer at rest in W' than } \\
\text { when measured by an observer at } \\
\text { rest in W. } \\
\text { This clock (or clocks) that is (are) } \\
\text { measured and that is (are) of } \\
\text { identical construction to the clocks } \\
\text { at rest in W that are used to measure } \\
\text { the clock (or clocks) at rest in W' is } \\
\text { in Scenario 2, instead, used to } \\
\text { measure the rate of the latter clocks } \\
\text { (the clocks at rest in W) where the } \\
\text { latter clocks are the "moving" } \\
\text { clocks. }\end{array}$ \\
\hline $\begin{array}{l}\text { State of Motion of } \\
\text { Reference Frame } \\
\text { for Observer at } \\
\text { Rest in That } \\
\text { Frame: }\end{array}$ & $\begin{array}{l}\text { "Stationary" for observer at rest in } \\
\text { W. }\end{array}$ & $\begin{array}{l}\text { "Stationary" for observer at rest in } \\
\text { W'. }\end{array}$ \\
\hline $\begin{array}{l}\text { State of Motion of } \\
\text { Reference Frame } \\
\text { for Observer at } \\
\text { Rest in Other } \\
\text { Frame: }\end{array}$ & $\begin{array}{l}\text { "Moving" for observer at rest in } \\
\text { W'. }\end{array}$ & "Moving" for observer at rest in $\mathrm{W}$. \\
\hline
\end{tabular}




\section{The Association}

\begin{tabular}{|c|c|c|}
\hline \multicolumn{3}{|c|}{$\begin{array}{c}\text { TABLE 2: } \\
\text { SCENARIO } 2\end{array}$} \\
\hline $\begin{array}{l}\text { Inertial Reference } \\
\text { Frame: }\end{array}$ & $\mathrm{W}$ & $\mathrm{W}^{\prime}$ \\
\hline $\begin{array}{l}\text { Logical Role in } \\
\text { Derivation of } \\
\text { Relativity of } \\
\text { Simultaneity: }\end{array}$ & $\begin{array}{l}\text { "Moving" frame. } \\
\text { Time in W' used to determine } \\
\text { whether criterion for simultaneity } \\
\text { met in W (using same light used to } \\
\text { establish simultaneity in } \mathrm{W}^{\prime} \text { ). }\end{array}$ & $\begin{array}{l}\text { "Stationary" frame. } \\
\text { Simultaneity established first in } \\
\text { this frame. }\end{array}$ \\
\hline $\begin{array}{l}\text { State of Motion of } \\
\text { Rod Measured in } \\
\text { Equation 3: }\end{array}$ & $\begin{array}{l}\text { Rod measured by observer at rest in } \\
\mathrm{W}^{\prime} \text { is at rest in W. } \\
\text { The length of this rod at rest in } \mathrm{W} \\
\text { is longer when measured by an } \\
\text { observer at rest in W than when } \\
\text { measured by an observer at rest in } \\
\mathrm{W}^{\prime} \text {. } \\
\text { This rod that is measured and that } \\
\text { is of identical construction to the } \\
\text { rod at rest in } \mathrm{W}^{\prime} \text { that is used to } \\
\text { measure the former rod in } \mathrm{W}^{\prime} \text { is in } \\
\text { Scenario } 1 \text {, instead, used to } \\
\text { measure the length of the latter rod } \\
\text { (the rod at rest in } \mathrm{W}^{\prime} \text { ) where the } \\
\text { latter rod is the "moving" rod. }\end{array}$ & $\begin{array}{l}\text { Rod measured by observer at rest in } \\
\mathrm{W}^{\prime} \text { is "moving" relative to } \mathrm{W}^{\prime} \text {. } \\
\Delta x^{\prime} \text { is determined for "moving" } \\
\text { measuring rod. Need to determine } \\
\text { positions of ends of rod in } \mathrm{W}^{\prime} \\
\text { simultaneously. } \\
\text { To measure } \Delta x^{\prime} \text {, observer at rest in } \\
\mathrm{W}^{\prime} \text { uses rod of identical } \\
\text { construction to the rod being } \\
\text { measured. This rod used to } \\
\text { measure } \Delta x^{\prime} \text { is at rest in W'. } \\
\text { The length of the rod "moving" in } \\
\text { W' is shorter when measured by an } \\
\text { observer at rest in W' than when } \\
\text { measured by an observer at rest in } \\
\text { W. }\end{array}$ \\
\hline
\end{tabular}




\section{Appendix A}

\begin{tabular}{|c|c|c|}
\hline \multicolumn{3}{|c|}{$\begin{array}{l}\text { TABLE } 2 \text { (cont'd) } \\
\text { SCENARIO 2: }\end{array}$} \\
\hline $\begin{array}{l}\text { Inertial Reference } \\
\text { Frame: }\end{array}$ & $\mathrm{W}$ & $W^{\prime}$ \\
\hline $\begin{array}{l}\text { State of Motion of } \\
\text { Clocks Measured } \\
\text { in Equation 1: }\end{array}$ & $\begin{array}{l}\text { Clock (or clocks) measured by } \\
\text { observer at rest in W' is at rest in } \\
\text { W. } \\
\text { The rate of this clock (or clocks) at } \\
\text { rest in W is faster when measured } \\
\text { by an observer at rest in W than } \\
\text { when measured by an observer at } \\
\text { rest in W'. } \\
\text { This clock (or clocks) that is (are) } \\
\text { measured and that is (are) of } \\
\text { identical construction to the clocks } \\
\text { at rest in W' that are used to } \\
\text { measure the clock (or clocks) at } \\
\text { rest in W is in Scenario } 1 \text {, instead, } \\
\text { used to measure the rate of the } \\
\text { latter clocks (the clocks at rest in } \\
\text { W') where the latter clocks are the } \\
\text { "moving" clocks. }\end{array}$ & $\begin{array}{l}\text { Clock (or clocks) measured by } \\
\text { observer at rest in W is "moving" } \\
\text { relative to W'. } \\
\text { Clocks at rest in } \mathrm{W}^{\prime} \text { are used to } \\
\text { determine } \Delta t^{\prime} \text { corresponding to } \Delta t \\
\text { in W. Observers at rest in } \mathrm{W}^{\prime} \text { use } \\
\text { clocks of identical construction to } \\
\text { that (or those) clock (or clocks) at } \\
\text { rest in W that is (or are) being } \\
\text { measured. } \\
\text { The rate of the clock (or clocks) } \\
\text { "moving" in W' is (or are) slower } \\
\text { when measured by an observer at } \\
\text { rest in W' than when measured by } \\
\text { an observer at rest in W. }\end{array}$ \\
\hline $\begin{array}{l}\text { State of Motion of } \\
\text { Reference Frame } \\
\text { for Observer at } \\
\text { Rest in That } \\
\text { Frame: }\end{array}$ & $\begin{array}{l}\text { "Stationary" for observer at rest in } \\
\text { W. }\end{array}$ & $\begin{array}{l}\text { "Stationary" for observer at rest in } \\
\text { W'. }\end{array}$ \\
\hline $\begin{array}{l}\text { State of Motion of } \\
\text { Reference Frame } \\
\text { for Observer at } \\
\text { Rest in Other } \\
\text { Frame: }\end{array}$ & $\begin{array}{l}\text { "Moving" for observer at rest in } \\
\text { W'. }\end{array}$ & $\begin{array}{l}\text { "Moving" for observer at rest in } \\
\text { W. }\end{array}$ \\
\hline
\end{tabular}




\section{Appendix B \\ The Nature of $c+v$ and \\ $c-v$ in the Special Theory}

Einstein explicitly acknowledged that $c-v$ is a velocity. As an example, in deriving the Lorentz transformation equations in his original paper on the special theory, Einstein discussed a "stationary" inertial system $\mathrm{K}$ and a "moving" inertial system $\mathrm{k}$ moving in a uniform translational manner relative to one another. Concerning a ray of light moving in the same direction that $\mathrm{k}$ is moving relative to K, Einstein (1905/1952) wrote:

But the ray [of light] moves relatively to the initial point of $\mathrm{k}$ [the "moving" system], when measured in the stationary system [K], with the velocity $c-v$, so that

$$
\frac{x^{\prime}}{c-v}=t \text { (p. 43). }
$$

$x^{\prime}$ is the distance traveled by the ray of light in $\mathrm{K}$, and $t$ is the time taken in $\mathrm{K}$ to traverse this distance with the velocity $c-\mathrm{v}$. In another translation of a large section of Einstein's paper, Schwartz (1977) translates the textual part of the above quote from Einstein as:

But, as measured in the stationary system, the ray moves with velocity $V-v$ relative to the origin of $\mathrm{k}$, so that we have

$$
x^{\prime} /(V-v)=t \text { (p. 21) }
$$

$V$ here is the invariant velocity of light in all inertial reference frames.

Because $c-v$ and $c+v$ are not measurable velocities of light, many physicists maintain that it is incorrect to consider $c-v$ and $c+v$ velocities of light. It is the invariant velocity of light in inertial reference frames that is measurable, and this is what both the "stationary" observer and the "moving" observer measure. Instead of using $c-v$ and $c+\mathrm{v}$, one may use distances to derive the relativity of simultaneity. More specifically, in arguing the relativity of simultaneity along the lines of Einstein's 1905 argument, for example, distances traveled by the light ray on its path from one end of the moving rod, A, to the other end, B, and its return from B to A may be used. The distances are those which an observer in the "stationary" inertial reference frame determines are the distances the light ray travels relative to an observer at rest in the "moving" inertial frame. These different distances determined by the 


\section{Appendix B}

"stationary" observer, though, are determined using the velocities $c$ and $v$, the very components that make up $c-v$ and $c+v$.

Though these distances are measurable by the "stationary" observer, they are no more real for the "moving" observer than are $c-v$ and $c+v$ as the velocities of light for the "moving" observer. For the "stationary" observer, the values $c-v$ and $c+v$ are indeed actual velocities of the light ray relative to the "moving" observer. It is just that the "moving" observer will not measure the velocities of the light ray to be $c-v$ and $c+\mathrm{v}$. Instead, the "moving" observer will measure the value of the velocity of light to be $c$. It is really no different concerning the use of distance to derive the relativity of simultaneity. The distances used instead of $c-v$ and $c+v$ are those measurable by the "stationary" observer, and the derivation of these distances depends on the use of $v$ as well as c. The distances are calculated using the velocities $c$ and $v$ and the time in the "stationary" inertial frame.

But for the "moving" observer, who considers his inertial reference frame "stationary," the velocity of light is c relative to him and thus $v$ is not a factor for him in the calculation of distance traveled by light in his inertial frame. Empirical evidence supports the position of the "moving" observer. He will measure the velocity of light to be $c$, and he will correctly calculate the distance traveled by light by using the velocity $c$ and the time in his inertial frame. Thus, using distances instead of velocities to argue the relativity of simultaneity in order to minimize the significance of $c-v$ and $c+v$ does not work. 


\section{Appendix C \\ The Light Flashes Used \\ to Establish Simultaneity in Inertial Reference \\ Frames in the Special Theory}

There is an interesting circumstance that results from the possibility of arguing the relativity of simultaneity in either direction, that is with either of two inertial reference frames in uniform translational motion relative to one another designated the "stationary" frame while the other reference frame is designated the "moving" frame. The argument on the relativity of simultaneity affects the course in the physical world of the light flashes used by observers to establish simultaneity in their respective inertial reference frames. This circumstance serves as an additional indication that the arbitrary decision as to which direction to argue the relativity of simultaneity has an impact on the physical world itself. This effect occurs even though the physical existence of the light flashes themselves appears not to be impacted. It is the same light flashes that are used in either scenario chosen.

In the case of the train gedankenexperiment, the inertial reference frames are the embankment and the train. There is a set of four world lines (or, perhaps, more precisely, three world lines of which one has a shorter element) for defining simultaneity in the inertial reference frames of the embankment and the train in each of the two scenarios for arguing the relativity of simultaneity. There are two distinct sets, one for each scenario. The scenario in which the chosen direction has the reference frame of the embankment as the "stationary" inertial reference frame and the train as the "moving" inertial reference frame is depicted in Figure 9. In this scenario, the light flash emitted in event A and meeting in event $\mathrm{C}$ the observer at rest midway on the section of embankment (the end positions of which correspond to the ends of the "moving" train), and the light flash emitted in event $\mathrm{B}$ and meeting the light flash from $\mathrm{A}$ in $\mathrm{C}$ are used to establish simultaneity in the "stationary" inertial reference frame of the embankment. The light flash emitted in A that meets the observer at rest on the embankment in $\mathrm{C}$ and travels to event $\mathrm{D}$ where it meets the observer at rest midpoint on the train, and the light flash emitted in event $\mathrm{E}$ from the other end of the train that meets the light flash from $\mathrm{A}$ in $\mathrm{D}$, are used to establish simultaneity in the "moving" inertial reference frame of the train.

The reverse circumstance can be seen in Figure 11. Where the inertial reference frame of the train is the "stationary" frame, the light flash emitted in 


\section{Appendix C}

event A meets the observer at rest midway on the train in event $\mathrm{H}$, the ends of the train corresponding to the positions on the "moving" embankment where the lightning flashes struck. The light flash emitted in event $\mathrm{G}$ meets the light flash from $\mathrm{A}$ in event $\mathrm{H}$. These two light flashes are used to establish simultaneity in the "stationary" inertial reference frame of the train. The light flash emitted in A meets the observer at rest midpoint on the embankment in event I. The light flash emitted in event $\mathrm{F}$, from the other end of the embankment where lightning struck, meets the light flash from A in I. These two light flashes are used to establish simultaneity in the "moving" inertial reference frame of the embankment. The embankment is "moving" in the direction of decreasing values of $x^{\prime}$ and thus toward the light flash emitted in A. This light flash, in terms of the time of the "stationary" reference frame of the train, meets the observer midpoint on the embankment in I before the light flash reaches the observer at rest midpoint on the train in $\mathrm{H}$.

It has been shown that it is an arbitrary decision which frame is considered the "stationary" reference frame and which the "moving" reference frame in Einstein's argument on the relativity of simultaneity. Thus, the question arises: What is the relationship between the light flashes from $\mathrm{A}$ to $\mathrm{C}$ in Figure 9 and from A to $\mathrm{H}$ in Figure 11, as well as those light flashes from B to $\mathrm{C}$ in Figure 9 and from $\mathrm{G}$ to $\mathrm{H}$ in Figure 11, that is between the light flashes that are used to establish simultaneity in the reference frames of the embankment and train respectively when each is considered the "stationary" inertial reference frame in the argument on the relativity of simultaneity? The light flashes from A to $\mathrm{C}$ and from $\mathrm{B}$ to $\mathrm{C}$ can have logical priority in the argument, or the light flashes from $\mathrm{A}$ to $\mathrm{H}$ and from $\mathrm{G}$ to $\mathrm{H}$ can have logical priority. In terms of the argument, there is only this logical distinction that is arbitrarily decided.

Are the light flashes from $\mathrm{A}$ to $\mathrm{C}$ and from $\mathrm{A}$ to $\mathrm{H}$ the same light flash? Or are the light flashes from $\mathrm{B}$ to $\mathrm{C}$ and from $\mathrm{G}$ to $\mathrm{H}$ the same light flash? For that matter are the light flashes from A to D in Figure 9 and from A to I in Figure 11 the same? And are the light flashes E to D in Figure 9 and from F to I in Figure 11 the same? As can be seen by their world lines in Figures 9 and 11, the members of each pair appear to be different flashes of light in the context of their roles in one of the scenarios in the argument on the relativity of simultaneity (i.e., with one inertial reference frame designated the "stationary" reference frame and the other inertial reference frame designated the "moving" reference frame). But since the argument on the relativity of simultaneity can be made in either direction without physical constraint and thus with the same light 


\section{The Light Flashes}

flashes, it would seem that the light flashes from $\mathrm{A}$ to $\mathrm{C}$ and from $\mathrm{A}$ to $\mathrm{H}$ are indeed the same light flash, the light flashes from $B$ to $C$ and from $G$ to $H$ are the same light flash, the light flashes from A to D and from A to I are the same light flash, and the light flashes from $\mathrm{E}$ to $\mathrm{D}$ and from $\mathrm{F}$ to I are the same light flash. One might expect that if this is the case, instead of two world lines for each of the light flashes that play the same role in the argument on the relativity of simultaneity, albeit in different scenarios, there would be only one world line for the light flashes. Instead, the argument on the relativity of simultaneity indicates that a light flash involved in establishing simultaneity in an inertial reference frame has two world lines in the special theory. 


\section{Appendix D}

\section{Light Clocks and Non-Light Clocks}

There is another way to approach the link between cognition and the physical world in the special theory. It has been discussed that in the special theory time is dependent on the motion of light. It would seem then that clocks at rest in an inertial reference frame that use some periodic phenomenon not based on the motion of light should still keep time in accord with the results of the special theory of relativity. This is what the experimental evidence supports, and it is an unusual consequence of the special theory. Regarding this feature of the special theory, Feynman, Leighton, and Sands (1963) wrote about a clock that keeps time based on the periodic motion of light and that is moving with a uniform translational velocity:

Not only does this particular kind of clock [i.e., the light clock] run more slowly, but if the theory of relativity is correct, any other clock, operating on any principle whatsoever, would also appear to run slower, and in the same proportion-we can say this without further analysis. Why is this so?

To answer the above question, suppose we had two other clocks made exactly alike with wheels and gears, or perhaps based on radioactive decay, or something else. Then we adjust these clocks so they both run in precise synchronism with our first [light] clocks [which are originally all at rest in one inertial reference frame]. When light goes up and back in the first [light] clocks and announces its arrival [back at the light source] with a click, the new [non-light] models also complete some sort of cycle, which they simultaneously announce by some doubly coincident flash, or bong, or other signal. One of these clocks is taken into the space ship [the "moving" inertial reference frame] along with the first kind [i.e., the light clock]. Perhaps this clock will not run slower, but will continue to keep the same time as its stationary counterpart, and thus disagree with the other moving clock [the light clock]. Ah no, if that should happen, the man in the ship could use this mismatch between his two clocks to determine the speed of his ship, which we have been supposing is impossible [because of the principle of relativity]. We need not know anything about the machinery of 


\section{Light Clocks}

the new clock that might cause the effect-we simply know that whatever the reason, it will appear to run slow, just like the first one.

Now if all moving clocks run slower, if no way of measuring time gives anything but a slower rate, we shall just have to say, in a certain sense, that time itself appears to be slower in a space ship. All the phenomena there-the man's pulse rate, his thought processes, the time he takes to light a cigar, how long it takes to grow up and get old-all these things must be slowed down in the same proportion, because he cannot tell he is moving [italics added]. The biologists and medical men sometimes say it is not quite certain that the time it takes for a cancer to develop will be longer in a space ship, but from the viewpoint of a modern physicist it is nearly certain; otherwise one could use the rate of cancer development to determine the speed of the ship! (p. 15-6)

Essentially, Feynman et al. invoked the postulate of special relativity that the laws of physics are the same in inertial reference frames in uniform translational motion relative to one another to account for the lack of difference between light clocks and no-light clocks. Feynman et al. wrote:

The principle of relativity was first stated by Newton, in one of his corollaries to the laws of motion: "The motions of bodies included in a given space are the same among themselves, whether that space is at rest or moves uniformly forward in a straight line." This means, for example that if a space ship is drifting along at a uniform speed, all experiments performed in the space ship and all the phenomena in the space ship will appear the same as if the ship were not moving, provided, of course, that one does not look outside. (p. 15-1)

Feynman et al. noted that the change in the principle of relativity found in the special theory, as opposed to its formulation in Newtonian mechanics, is:

all physical laws [including those for electromagnetism] should be of such a kind that they remain unchanged under a Lorentz transformation [instead of a Galilean transformation]. In other words, we should change, not the laws of electrodynamics, but the laws of mechanics. (p. 15-3) 


\section{Appendix D}

If other periodic phenomena in inertial reference frames did not follow the concept of time exemplified by the light clock, it is possible there would be temporal differences in these inertial reference frames such that the laws of physics would not hold in all such frames. It is central to the special theory to maintain the integrity of this postulate of relativity. There is no identifiable physical basis for this phenomenon of other periodic occurrences holding to the concept of time rooted fundamentally in the relativity of simultaneity that Einstein developed using the motion of light.

The question is: how does light affect periodic phenomena of which it is not an essential aspect? The answer is: no mechanism for this effect has been described because there is no physical effect to account for. It is easy to conceive of an experiment in which a clock is used that in no way incorporates light and in which light is excluded from the physical circumstances of the experiment. Why then does this clock function in these circumstances in accord with the concept of time that in the special theory is established using the motion of light? How is it possible that clocks at rest in an inertial reference frame that use some periodic phenomenon not based on the motion of light should still keep time in accordance with non-existent light clocks?

Support in the special theory for a cognitive component to light and also for a cognitive component to time in an inertial reference frame is the basis for Feynman et al.'s supposition that the theoretical integrity of the special theory depends on non-light clocks functioning in the same manner as do light clocks. The special theory of relativity is, of course, supported by a great deal of empirical evidence. But, for all this work, none of it has addressed the fact that there are no known physical forces that can account for the influence of light and light clocks on non-light clocks that is implied in the establishment of time in inertial reference frames in the special theory. There is only this requirement to maintain the theoretical integrity of the special theory.

What is the nature of such a requirement without an underlying physical explanation? A theory is formulated by individuals to explain something. In the case of the special theory, its role is to explain certain fundamental aspects of the functioning of the physical world. A theory may be tested in empirical terms, and it may, as in the case of the special theory, have a great deal of empirical support. If a theory has a great deal of empirical support, it may reasonably be thought that the theory reflects to a high degree the functioning of that which it is attempting to explain. In large part, this is the case with the special theory in its explanation of the functioning of the physical world. But 


\section{Light Clocks}

where a theory, such as the special theory, does not provide an explanation of that which it is supposed to explain, and instead relies on the structure of the theory itself for how that phenomenon to be explained functions, then the theory itself is related to that which it is supposed to explain.

If that which is explained is the physical world, and the theory is supported by empirical evidence in the physical world, then the theory is linked directly to the physical world. This is the case with the effect of light on the functioning of non-light clocks in the special theory. This effect of light is mediated by the theoretical requirement of the special theory that the laws of physics hold in inertial reference frames in uniform translational motion relative to one another. Thus, the special theory, a cognitive construction, is linked directly to the physical world (Snyder, 1992b). 


\section{Appendix E \\ The Mathematical Representation of Thermal Equilibrium}

The temperature of each of the component systems is given by

$$
1 / T=k_{B}(\partial \sigma / \partial U)_{N}
$$

where $T$ is the temperature given in degrees Kelvin, $\sigma$ is the entropy of the component system, $U$ is the energy of the system, $N$ is the number of particles in the system, and $k_{B}$ is the Boltzmann constant. The symbol $\partial$ indicates partial differentiation and thus the term $(\partial \sigma / \partial U)_{N}$ indicates the partial differentiation of the entropy with respect to the energy, while the number of particles in the system is held constant. Roughly put, this partial differentiation is the ratio of the rate of change of the entropy to the rate of change of the energy of the system. As noted, the entropy, $\sigma$, is the natural logarithm of the number of accessible states for a specified physical system. Because of the fundamental assumption, $\sigma$ is a measure of the disorder in the system.

The condition of thermal equilibrium is given by

$$
k_{B}\left(\partial \sigma_{1} / \partial U_{1}\right)_{N 1}=k_{B}\left(\partial \sigma_{2} / \partial U_{2}\right)_{N 2}
$$

or

$$
T_{1}=T_{2}
$$

where the subscript 1 indicates values of quantities in one of the component systems and the subscript 2 indicates values of quantities in the other component system. The entropy of the component systems toward which the systems tend is determined once the energy, the number of particles, and the volume of the overall physical system is specified. Small fluctuations in the energy of the component systems must meet equations 26 and 27 when these systems are in thermal equilibrium. 


\section{Appendix F \\ Tolman's Argument Concerning the Equivalence of Time and Ensemble Averages}

First, consider time averages for individual physical systems. If there are a number of systems like the isolated system of interest, all of them being very similar in energy, one assumes that each of the systems will spend equal amounts of time in the states accessible to the system of interest. Each system is assumed to show the same long term behavior. If this is the case, then $\mathrm{NaT}_{x}$ (28) represents the amount of time spent by all these systems in a particular state $x$, where $N$ is the total number of systems, $T$ is the long time interval over which the physical systems are considered, and $a_{x}$ is the fraction of the long time interval that any one of these systems is in the particular state $x$.

On the other hand, for the representative ensemble of systems like the physical system of interest with $N$ systems, where $N_{x}$ is the number of systems in state $x$ at any time $t$

$$
\int_{0}^{T} N_{x} d t
$$

represents the number of systems in this ensemble in the state x over the time $T$, the same time interval noted above. Then

$$
N a_{x} T=\int_{0}^{T} N_{x} d t .
$$

This relationship holds for any accessible state. Since the states of the system in the representative ensemble do not change over time

$$
\int_{0}^{T} N_{x} d t=N_{x} \int_{0}^{T} d t .
$$

Then 


\section{Appendix F}

$$
N_{x} \int_{0}^{T} d t=N_{x} T
$$

and

$$
a_{x}=N_{x} / N
$$

That is, in the time averaging scenario, the fraction of the total time spent in any one of the accessible states by the single system or similar system in the time averaging scenario is equal to the number of systems in the representative ensemble in that state divided by the total number of systems in the representative ensemble.

Although elegant, there are significant problems with this argument. Tolman pointed out that in the case of the time average for a single system, there is no principle that provides the conceptual support for the basic premise of the argument that the time average of a single system is equivalent to the average of the representative ensemble at a particular time. This basic premise is that the single system will pass through every accessible state over time before returning to its original state. This premise, after Boltzmann, is known as the ergodic hypothesis. ${ }^{17}$

For example, it is possible that a single system might remain in a particular state and not occupy all accessible states in the particular manner indicated. The assumption that a single system will spend equal amounts of time in all accessible states is, in Tolman's terms, fundamentally a statistical principle. It is not derived from the laws of mechanics that precisely prescribe the development in time and space of physical systems.

Moreover, Tolman argued that the time period required to obtain a time average for a single system in arguing the equivalence of this average with that obtained for the representative ensemble is much greater than the amount of time that elapses in an experiment. To assume that the time average of a single system obtained over a short period of time would be the same as that obtained

\footnotetext{
${ }^{17}$ Tolman (1938) stated the ergodic hypothesis this way: "It [the ergodic hypothesis] states that the phase point for any isolated system would pass in succession through every point compatible with the energy of the system before finally returning to its original position in the phase space" (p. 65).
} 


\section{Tolman's Argument}

over a long period of time is essentially a statistical assumption, not one grounded in lawful processes of the physical world.

The ergodic hypothesis, which was in part apparently developed in an attempt to preserve the role of the laws of mechanics in thermodynamics, does not have the necessary conceptual breadth to accomplish this task. Thus, as Tolman argued, thermodynamics is fundamentally statistical in nature, meaning that for a representative ensemble of systems like the system of interest, any one of these accessible states of the system of interest is equally likely. The representative ensemble that is central to statistical mechanics consists of imagined systems similar to the real system of interest, but upon which measurements of thermodynamic quantities depend on no less than the real system.

In the first edition of Thermal Physics, Kittel (1969) follows in the footsteps of Maxwell and Boltzmann, acknowledging the significance of the representative ensemble but nonetheless maintaining some allegiance to the time average for a single system. Tolman (1938) wrote:

In the course of the historical development of statistical mechanics, the above point of view as to the validity of its methods [relying on the representative ensemble and the fundamental assumption] was not the one ultimately adopted by Maxwell and Boltzmann. With the help of a different assumption [the ergodic hypothesis], rather than our own bald postulate as to equal a priori probabilities, it was hoped to justify the methods of statistical mechanics by showing that the time average of any quantity pertaining to any single system of interest would actually agree with the ensemble average for that quantity calculated by the methods of statistical mechanics for all members of the corresponding representative ensemble. (p. 65)

As Tolman has shown, the time average for an individual system relying on the ergodic hypothesis is not equivalent to the ensemble average. 


\section{References}

Bennett, C. H. (1987). Demons, engines and the second law. Scientific American, 257(5), 108-116.

Bergquist, J. C., Hulet, R. G., Itano, W. M., and Wineland, D. J. (1986). Observation of quantum jumps in a single atom. Physical Review Letters, 57, 1699-1702.

Bohr, N. (1935). Can quantum-mechanical description of nature be considered complete? Physical Review, 49, 1804-1807.

Bohr, N. (1961). Atomic theory and the description of nature. Cambridge, England: Cambridge University Press. (Original work published 1934)

Bohr, N. (1969). Discussion with Einstein on epistemological problems in atomic physics. In P. A. Schilpp (Ed.), Albert Einstein: Philosopher-scientist (Vol. 1) (pp. 199-241). La Salle, Illinois: Open Court. (Original work published 1949)

Boring, E. G. (1950). A history of experimental psychology (2nd ed.). New York: AppletonCentury-Crofts. (Original work published 1929)

Born, M. (1965). Einstein's theory of relativity (rev. ed.) New York: Dover. (Original work published 1924)

Brewer, R. G., and Hahn, E. L. (1984). Atomic memory. Scientific American, 251(6), 50-57.

Brown, G. G. (1928). Perception of depth with disoriented vision. The British Journal of Psychology - General Section, 19, 117-146.

Cohen, I.B. (1981). Newton's discovery of gravity. Scientific American, 244(3), 166-179.

Cook, R. J. (1990). Quantum jumps. In E. Wolf (Ed.), Progress in Optics (Vol. 28) (pp. 361-416). Amsterdam: North-Holland.

Cooper, L. A. (1975). Mental rotation of random two-dimensional shapes. Cognitive Psychology, 7, 20-43.

Cooper, L. A. (1976). Demonstration of a mental analog of an external rotation. Perception \& Psychophysics, 19, 296-302.

Cooper, L. A., and Podgorny, P. (1976). Mental transformations and visual comparison processes. Journal of Experimental Psychology: Human Perception and Performance, 2, 503-514.

Cooper, L. A., and Shepard, R. N. (1984). Turning something over in the mind. Scientific American, 251(6), 106-114.

Dicke, R. H., and Wittke, J. P. (1960). Introduction to quantum mechanics. Reading, Massachusetts: Addison-Wesley.

Dolezal, H. (1982). Living in a world transformed. New York: Academic Press.

Einstein, A. (1952). On the electrodynamics of moving bodies. In H. Lorentz, A. Einstein, H. Minkowski, and $\mathrm{H}$. Weyl (Eds.), The principle of relativity, a collection of original memoirs on the special and general theories of relativity (pp. 35-65) (W. Perrett and G. B. Jeffrey, Trans.). New York: Dover. (Original work published 1905)

Einstein, A. (1952). The foundation of the general theory of relativity. In H. Lorentz, A. Einstein, $\mathrm{H}$. Minkowski, and $\mathrm{H}$. Weyl (Eds.), The principle of relativity, a collection of original memoirs on the special and general theories of relativity (pp. 109-164)

(W. Perrett and G. B. Jeffrey, Trans.). New York: Dover. (Original work published 1916) 


\section{References}

Einstein, A. (1956). The meaning of relativity (5th ed.) (E. Adams, Trans.). Princeton, New Jersey: Princeton University Press. (Original work published 1922)

Einstein, A. (1961). Relativity, the special and the general theory (R. Lawson, Trans.). New York: Bonanza. (Original work published 1917)

Einstein, A. (1969). Autobiographical notes. In P. A. Schilpp (Ed.), Albert Einstein: Philosopher-scientist (Vol. 1) (pp. 1-94). La Salle, Illinois: Open Court. (Original work published 1949)

Einstein, A. (1993). The principle of relativity and its consequences in modern physics. In The collected papers of Albert Einstein (Vol. 3) (pp. 117-142) (A. Beck, Trans.). Princeton, New Jersey: Princeton University Press. (Original work published 1910)

Einstein, A., and Infeld, L. (1966). The evolution of physics from early concepts to relativity and quanta. New York: Simon and Schuster. (Original work published 1938)

Einstein, A., Podolsky, B., and Rosen, N. (1935). Can quantum-mechanical description of physical reality be considered complete? Physical Review, 47, 777-780.

Eisberg, R., and Resnick, R. (1985). Quantum physics of atoms, molecules, solids, nuclei and particles (2nd ed.). New York: Wiley. (Original work published 1974)

Epstein, P. (1945). The reality problem in quantum mechanics. American Journal of Physics, 13, 127-136.

Erismann, T., and Kohler, I. (1953). Upright vision through inverting spectacles [Film]. University Park, Pennsylvania: PCR: Films and Video in the Behavioral Sciences.

Erismann, T., and Kohler, I. (1958). Living in a reversed world [Film]. University Park, Pennsylvania: PCR: Films and Video in the Behavioral Sciences.

Ewert, P. W. (1930). A study of the effect of inverted retinal stimulation upon spatially coordinated behavior. Genetic Psychology Monographs: Child Behavior, Animal Behavior, and Comparative Psychology, 7, 177-363.

Feynman, R. P. (1982). Simulating physics with computers. International Journal of Theoretical Physics, 21, 467-488.

Feynman, R. P., Leighton, R. B., and Sands, M. (1963). The Feynman lectures on physics: Mainly mechanics, radiation, and heat (Vol. 1). Reading, Mass.: Addison-Wesley.

Feynman, R. P., Leighton, R. B., and Sands, M. (1964). The Feynman lectures on physics: Mainly electromagnetism and matter (Vol. 2). Reading, Mass.: Addison-Wesley.

Feynman, P. R., Leighton, R. B., and Sands, M. (1965). The Feynman lectures on physics: Quantum mechanics (Vol. 3). Reading, Massachusetts: Addison-Wesley.

Gasiorowicz, S. (1974). Quantum physics. New York: John Wiley.

Georgopoulos, A. P., Lurito, J. T., Petrides, M., Schwartz, A. B., and Massey J. T. (1989). Mental rotation of the neuronal population vector. Science, 243, 234-236.

Gibson, J. J. (1933). Adaptation, after-effect and contrast in the perception of curved lines. Journal of Experimental Psychology, 16, 1-31.

Goswami, A. (1989). The idealistic interpretation of quantum mechanics. Physics Essays, 2, 385-400.

Goswami, A. (1992). Quantum mechanics. Dubuque, Iowa: Wm. C. Brown.

Held, R. (1965). Plasticity in sensory-motor systems. Scientific American, 213(5), 84-94. 


\section{References}

Held, R., and Freedman, S. J. (1963). Plasticity in human sensorimotor control. Science, 142, 455-462.

Kandel, E. R., Schwartz, J. H., and Jessell, T. M. (1991). Principles of neural science (3rd edition). New York: Elsevier.

Kant, I. (1966). Critique of pure reason (F. Max Muller, Trans.). Garden City, New York: Anchor Books. (Original work published 1781)

Kittel, C. (1969). Thermal physics. New York: John Wiley \& Sons.

Kittel, C., and Kroemer, H. (1980). Thermal physics (2nd ed.). New York: W. H. Freeman. (Original work published 1969)

Kohler, I. (1962). Experiments with goggles. Scientific American, 206(5), 62-72.

Kohler, I. (1964). The formation and transformation of the perceptual world (H. Fiss, Trans.). Psychological Issues, 3, 19-133 and 165-173.

Liboff, R. (1993). Introductory quantum mechanics (2nd ed.). Reading, Massachusetts: Addison-Wesley.

Lurito, J. T., Georgakopoulos, T., and Georgopoulos, A. P. (1991). Cognitive spatial-motor processes: 7. The making of movements at an angle from a stimulus direction: Studies of motor cortical activity at the single cell and population levels. Experimental Brain Research, 87, 562-580.

Maxwell, J. C. (1865). A dynamical theory of the electromagnetic theory. Philosophical Transactions of the Royal Society of London, 155, 459-512.

Mermin, N. D. (1985, April). Is the moon there when nobody looks? Reality and the quantum theory. Physics Today, 38-47.

Merzbacher, E. (1970). Quantum mechanics (2nd. ed.). New York: John Wiley. (Original work published 1961)

Messiah, A. (1965). Quantum mechanics (2nd ed.) (Vol. 1) (G. Tremmer, Trans.). Amsterdam: North-Holland. (Original work published 1962)

Misner, C. W., Thorne, K. S., and Wheeler, J. A. (1973). Gravitation. San Francisco: W. H. Freeman.

Munn, N. L. (1965). The evolution and growth of human behavior (2nd edition). Boston: Houghton Mifflin. (Original work published 1955)

Nagourney, W., Sandberg, J., and Dehmelt, H. (1986). Shelved optical electron amplifier: observation of quantum jumps. Physical Review Letters, 56, 2797-2799.

Newton, I. (1962). Sir Issac Newton's mathematical principles of natural philosophy and his system of the world (Vols. 1-2) (A. Motte and F. Cajori, Trans.). Berkeley: University of California. (Original work published 1686)

Pellizzer, G., and Georgopoulos, A. P. (1993). Mental rotation of the intended direction of movement. Current Directions in Psychological Science, 2, 12-17.

Pronko, N. H., and Snyder, F. W. (1951). Vision with spatial inversion [Film]. University Park, Pennsylvania: PCR: Films and Video in the Behavioral Sciences.

Renninger, M. (1960). Messungen ohne Störung des Meßobjekts [Observations without disturbing the object]. Zeitschrift für Physik, 158, 417-421.

Resnick, R. (1968). Introduction to special relativity. New York: John Wiley and Sons. 


\section{References}

Sauter, T., Neuhauser, W., Blatt, R. and Toschek, P. E. (1986). Observation of quantum jumps. Physical Review Letters, 57, 1696-1698.

Schrödinger, E. (1983). The present situation in quantum mechanics. In J. A. Wheeler and W. H. Zurek, Quantum theory and measurement (pp. 152-167) (J. Trimmer, Trans.). Princeton, New Jersey: Princeton University Press. (Original work published 1935)

Schwartz, H. M. (1977). Einstein's first paper on relativity. American Journal of Physics, 45, $18-25$.

Shankland, R. S. (1964). The Michelson-Morley experiment. Scientific American, 211(5), 107-114.

Shepard, R. N., and Metzler, J. (1971). Mental rotation of three-dimensional objects. Science, 171, 701-703.

Snyder, D. M. (1983). On the nature of relationships involving the observer and the observed phenomenon in psychology and physics. The Journal of Mind and Behavior, 4, 389-400.

Snyder, D. M. (1985). On the conservation of physical quantities. Perceptual and Motor Skills, 60, 754.

Snyder, D. M. (1986). Light as an expression of mental activity. The Journal of Mind and Behavior, 7, 567-584.

Snyder, D. M. (1987). On temporal irreversibility. Speculations in Science and Technology, $10,221-223$.

Snyder, D. M. (1989). The inclusion in modern physical theory of a link between cognitiveinterpretive activity and the structure and course of the physical world. The Journal of Mind and Behavior, 10, 153-172.

Snyder, D. M. (1990). On the relation between psychology and physics. The Journal of Mind and Behavior, 11, 1-17.

Snyder, D. M. (1992a). Being at rest. The Journal of Mind and Behavior, 13, 157-161.

Snyder, D. M. (1992b). On light clocks and periodic phenomena in the special theory of relativity. Manuscript submitted for publication.

Snyder, D. M. (1992c). Quantum mechanics and the involvement of mind in the physical world: A response to Garrison. The Journal of Mind and Behavior, 13, 247-257.

Snyder, D. M. (1993). Quantum mechanics is probabilistic in nature. The Journal of Mind and Behavior, 14, 145-153.

Snyder, D. M. (1994). On the arbitrary choice regarding which inertial reference frame is "stationary" and which is "moving" in the special theory of relativity. Physics Essays, 7, 297-334.

Snyder, D. M. (1995). On the quantum mechanical wave function as a link between cognition and the physical world: A role for psychology. The Journal of Mind and Behavior, 16, 151-179.

Snyder, D. M. (1996a). Cognition and the physical world in quantum mechanics. Paper presented at the annual convention of the Western Psychological Association, San Jose, California.

Snyder, D. M. (1996b). On the nature of the change in the wave function in a measurement in quantum mechanics. Los Alamos National Laboratory E-Print Physics Archive (WWW address: http://xxx.lanl.gov/abs/quant-ph/9601006). 


\section{References}

Snyder, F. W., and Pronko, N. H. (1952). Vision with spatial inversion. Witchita, Kansas: University of Witchita Press.

Snyder, F. W., and Snyder, C. W. (1957). Vision with spatial inversion: A follow-up study. The Psychological Record, 7, 21-30.

Stratton, G. M. (1896). Some preliminary experiments on vision without inversion of the retinal image. The Psychological Review, 3, 611-617.

Stratton, G. M. (1897a). Vision without inversion of the retinal image - 1. The Psychological Review, 4, 341-360.

Stratton, G. M. (1897b). Vision without inversion of the retinal image - 2.

The Psychological Review, 4, 463-481.

Stratton, G. M. (1899). The spatial harmony of touch and sight. Mind: A Quarterly Review, $8,492-505$.

Tolman, R. C. (1938). The principles of statistical mechanics. London: Oxford University Press.

Wigner, E. (1983). Remarks on the mind-body question. In J. A. Wheeler and W. H. Zurek, Quantum theory and measurement (pp. 168-181). Princeton, New Jersey: Princeton University Press. (Original work published 1961) 